\author{
Universidade de São Paulo \\ Instituto de Física de SÃo Carlos \\ Departamento de Física e Informática
}

Michelle Matos Horta

\title{
Modelos de mistura de distribuições na segmentação de imagens SAR polarimétricas multi-look
}


Michelle Matos Horta

\section{Modelos de mistura de distribuições na segmentação de imagens SAR polarimétricas multi-look}

Tese apresentada ao Programa de Pós-Graduação em Física do Instituto de Física de São Carlos da Universidade de São Paulo, para obtenção do título de Doutor em Ciências.

Área de Concentração: Física Aplicada Opção Física Computacional Orientador: Prof. Dr. Nelson Delfino d'Ávila Mascarenhas

Co-orientador: Prof. Dr. Luciano da Fontoura Costa 


\begin{abstract}
AUTORIZO A REPRODUÇÃO E DIVULGAÇÃO TOTAL OU PARCIAL DESTE TRABALHO, POR QUALQUER MEIO CONVENCIONAL OU ELETRÔNICO, PARA FINS DE ESTUDO E PESQUISA, DESDE QUE CITADA A FONTE.
\end{abstract}

Ficha catalográfica elaborada pelo Serviço de Biblioteca e Informação IFSC/USP

Horta, Michelle Matos.

Modelos de mistura de distribuições na segmentação de imagens SAR polarimétricas multi-look / Michelle Matos Horta; orientador Nelson Delfino d'Ávila Mascarenhas; co-orientador Luciano da Fontoura Costa.-- São Carlos, 2009. $115 \mathrm{p}$.

Tese (Doutorado em Ciências - Área de concentração: Física Aplicada - Opção Física Computacional) - Instituto de Física de São Carlos da Universidade de São Paulo.

1. Imagens SAR polarimétricas multi-look. 2. Modelos de Mistura de Distribuições. 3. Algoritmo SEM. 4. Segmentação de Imagens I. Título. 


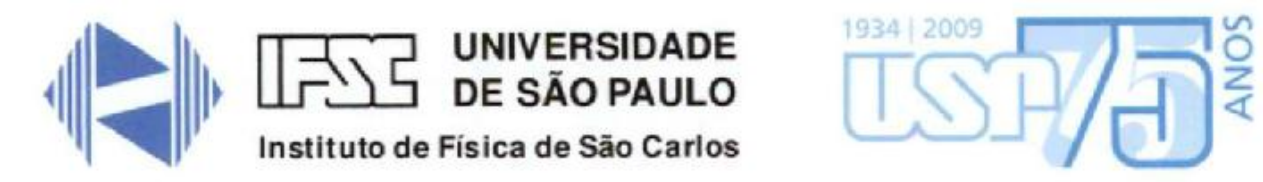

Caixa Postal 369

13560-970 São Carlos, SP

Av. Trabalhador São-carlense, 400

13566-590 - Sào Carlos, SP

Fone/Fax: (16) 3373.9777

www.ifse,usp.br

svposgrad@ifsc.usp.br

FOLHA DE APROVAÇÃO

Michelle Matos Horta

Tese apresentada ao Instituto de Física de Săo

Carlos da Universidade de Săo Paulo para obtenção do título de Doutor em Ciências. Área de Concentração: Fisica Aplicada - Opção: Fisica Computacional.

Aprovada em: 04/06/2009

Comissão Julgadora

Prof. Dr. Nelson Delfino D'Ávila Mascarenhas

Instituiçăo: UFSCar

Assinatura

Dr. Mateus Jose MArtins

Instituição: IFSC/USP

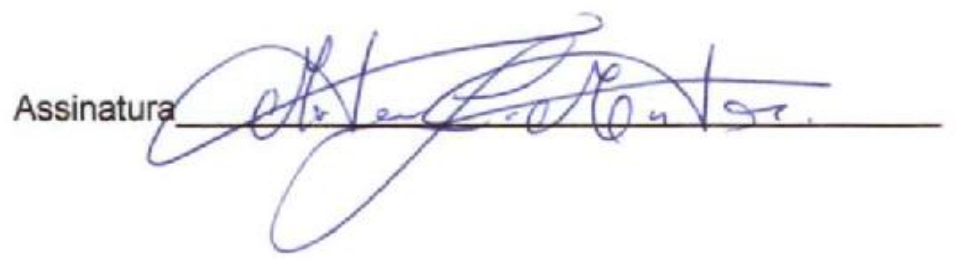

Prof. Dr. Elbert Einstein Nehrer Macau

Instituição: INPE

Assinatura

macauns

Prof. Dr. Alejandro César Frery Orgambide

Instituição: UFAL

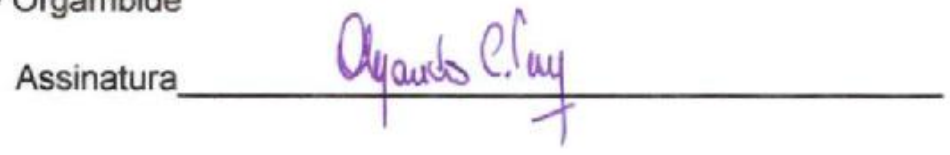

Profa. Dra. Corina da Costa Freitas

Instituição: INPE

Assinatura ePuras. 

Aos meus pais, Wiliam e Gleice.

Ao meu noivo, Anderson.

À minha família. 



\section{Agradecimentos}

Agradeço a todos que, direta ou indiretamente, me apoiaram neste trabalho.

Ao meu orientador, Dr. Prof. Nelson Delfino d'Àvila Mascarenhas, pela orientação e dedicação à elaboração desta tese.

Ao meu co-orientador, Dr. Luciano da Fontoura Costa, pela oportunidade de fazer o doutorado no IFSC.

Ao colaborador, Dr. Alejandro César Frery Orgambide, pelo apoio nas pesquisas e, principalmente, pela amizade.

Aos meus pais, Wiliam e Gleice, pela força, dedicação, apoio e carinho.

Ao meu noivo, Anderson, pela dedicação, carinho e apoio.

À minha família que me apoia em todos os momentos de minha vida.

Aos meus amigos de Recife. Aos amigos que fiz durante este doutorado e que me apoiaram em todos estes anos em São Carlos: Débora, Giovana, Alexandre Levada, Talita, Nathália, Fernanda, Mônica, Ana Luísa, Denis, Moacir, Marcelo, Murillo, João, Sílvia, Linder, Bruno, Wilma, Susana, Sandra, Alessandra, Tiago, Tatiane, Marco, Júlio, Alexandre e Cristian, além de muitos outros, como os amigos do Grupo de Visão Cibernética e do Gapis.

À FAPESP pelo apoio financeiro.

À USP e ao IFSC pelo suporte no desenvolvimento do doutorado.

Aos funcionários do IFSC, principalmente, os da secretaria e biblioteca, que me ajudaram durante o programa de doutorado.

À UFSCar e ao departamento de computação, pelo suporte e infra-estrutura.

Ao Instituto de Computação da UFAL pelo suporte durante o estágio de seis meses em 2007.

A Deus pela vida que tenho. 


\section{Resumo}

HORTA, M. M. Modelos de mistura de distribuições na segmentação de imagens SAR polarimétricas multi-look. 2009. 115p. Tese (Doutorado) - Instituto de Física de São Carlos, Universidade de São Paulo, São Carlos, 2009.

Esta tese se concentra em aplicar os modelos de mistura de distribuições na segmentação de imagens SAR polarimétricas multi-look. Dentro deste contexto, utilizou-se o algoritmo SEM em conjunto com os estimadores obtidos pelo método dos momentos para calcular as estimativas dos parâmetros do modelo de mistura das distribuições Wishart, $\mathcal{K}_{P}$ ou $\mathcal{G}_{P}^{0}$. Cada uma destas distribuições possui parâmetros específicos que as diferem no ajuste dos dados com graus de homogeneidade variados. A distribuição Wishart descreve bem regiões com características mais homogêneas, como cultivo. Esta distribuição é muito utilizada na análise de dados SAR polarimétricos multi-look. As distribuições $\mathcal{K}_{P}$ e $\mathcal{G}_{P}^{0}$ possuem um parâmetro de rugosidade que as permitem descrever tanto regiões mais heterogêneas, como vegetação e áreas urbanas, quanto regiões homogêneas. Além dos modelos de mistura de uma única família de distribuições, também foi analisado o caso de um dicionário contendo as três famílias. Há comparações do método SEM proposto para os diferentes modelos com os métodos da literatura k-médias e EM utilizando imagens reais da banda L. O método SEM com a mistura de distribuições $\mathcal{G}_{P}^{0}$ forneceu os melhores resultados quando os outliers da imagem são desconsiderados. A distribuição $\mathcal{G}_{P}^{0}$ foi a mais flexível ao ajuste dos diferentes tipos de alvo. A distribuição Wishart foi robusta às diferentes inicializações. O método k-médias com a distribuição Wishart é robusto à segmentação de imagens contendo outliers, mas não é muito flexível à variabilidade das regiões heterogêneas. O modelo de mistura do dicionário de famílias melhora a log-verossimilhança do método SEM, mas apresenta resultados parecidos com os do modelo de mistura $\mathcal{G}_{P}^{0}$. Para todos os tipos de inicialização e grupos, a distribuição $\mathcal{G}_{P}^{0}$ predominou no processo de seleção das distribuições do dicionário de famílias.

Palavras-chave: Imagens SAR polarimétricas multi-look, Modelos de Mistura de Distribuições, Algoritmo SEM, Segmentação de Imagens. 


\section{Abstract}

\section{HORTA, M. M. Multi-look polarimetric SAR image segmentation using mixture}

models. 2009. 115p. Thesis (Doctoral) - Instituto de Física de São Carlos, Universidade de São Paulo, São Carlos, 2009.

The main focus of this thesis consists of the application of mixture models in multi-look polarimetric SAR image segmentation. Within this context, the SEM algorithm, together with the method of moments, were applied in the estimation of the Wishart, $\mathcal{K}_{P}$ and $\mathcal{G}_{P}^{0}$ mixture model parameters. Each one of these distributions has specific parameters that allows fitting data with different degrees of homogeneity. The Wishart distribution is suitable for modeling homogeneous regions, like crop fields for example. This distribution is widely used in multi-look polarimetric $\mathrm{SAR}$ data analysis. The distributions $\mathcal{K}_{P}$ and $\mathcal{G}_{P}^{0}$ have a roughness parameter that allows them to describe both heterogeneous regions, as vegetation and urban areas, and homogeneous regions. Besides adopting mixture models of a single family of distributions, the use of a dictionary with all the three family of distributions was proposed and analyzed. Also, a comparison between the performance of the proposed SEM method, considering the different models in real $L$-band images and two widely known techniques described in literature (k-means and EM algorithms), are shown and discussed. The proposed SEM method, considering a $\mathcal{G}_{P}^{0}$ mixture model combined with a outlier removal stage, provided the best classification results. The $\mathcal{G}_{P}^{0}$ distribution was the most flexible for fitting the different kinds of data. The Wishart distribution was robust for different initializations. The k-means algorithm with Wishart distribution is robust for segmentation of SAR images containing outliers, but it is not so flexible to variabilities in heterogeneous regions. The mixture model considering the dictionary of distributions improves the SEM method log-likelihood, but presents similar results to those of $\mathcal{G}_{P}^{0}$ mixture model. For all types of initializations and clusters, the $\mathcal{G}_{P}^{0}$ prevailed in the distribution selection process of the dictionary of distributions.

Keywords: Fully polarimetric SAR images, Finite Mixture Models, Stochastic ExpectationMaximization Algorithm, Image Segmentation. 


\section{Lista de Figuras}

Figura 1 - Método de Segmentação SEM proposto. . . . . . . . . . . . . . . . 62

Figura 2 - Imagens de referência da região de São Francisco. . . . . . . . . . . 68

Figura 3 - Imagens de referência da região de Munique. . . . . . . . . . . . . . 69

Figura 4 - Curvas de convergência da log-verossimilhança do método SEM com a imagem de São Francisco. . . . . . . . . . . . . . . . . . . . 73

Figura 5 - Curvas de convergência da log-verossimilhança e do parâmetro de intensidade dos métodos SEM e EM com a imagem Munique completa. 75

Figura 6 - Curvas de convergência da log-verossimilhança dos métodos SEM e EM com a imagem Munique parcial. . . . . . . . . . . . . . . 76

Figura 7 - Diferentes inicializações da imagem São Francisco contendo $g=3$ regiões. . . . . . . . . . . . . . . . . 77

Figura 8 - Segmentações da imagem São Francisco obtidas na iteração de convergência com a inicialização quantil e $g=3 . . . . .79$

Figura 9 - Segmentações da imagem São Francisco obtidas na iteração de maior log-verossimilhança com a inicialização quantil e $g=4 \ldots$. . . . . . 81

Figura 10 - Segmentações da imagem São Francisco obtidas na iteração 32 com a inicialização quantil e $g=4 \ldots$. . . . . . . . . . . . . . . 81

Figura 11 - Curvas de convergência da log-verossimilhança do método SEM com o dicionário de famílias e a imagem de São Francisco utilizando a inicialização aleatória.

Figura 12 - Segmentações da imagem São Francisco obtidas na iteração de convergência com método SEM com o dicionário de famílias e as diferentes inicializações com $g=3 \ldots \ldots$. . . . . . . . . . . 85

Figura 13 - Gráfico de dispersão dos canais de intensidade da imagem São Francisco. . . . . . . . . . . . . . . . . . 100

Figura 14 - Histograma da imagem $S P A N$ de São Francisco. . . . . . . . . . . 100

Figura 15 - Gráfico de dispersão dos canais de intensidade da imagem de Munique.101 
Figura 16 - Histograma da imagem $S P A N$ de Munique. . . . . . . . . . . . . . . 101

Figura 17 - Curvas de convergência dos parâmetros da imagem São Francisco com $g=3$ regiões e inicialização aleatória. . . . . . . . . . . . . . . 104

Figura 18 - Segmentações da imagem São Francisco com $g=3$ regiões utilizando a inicialização aleatória. . . . . . . . . . . . . . . . . . . . . 105

Figura 19 - Curvas de convergência dos parâmetros da imagem São Francisco com $g=3$ regiões e inicialização k-médias. . . . . . . . . . . . 106

Figura 20 - Segmentações da imagem São Francisco com $g=3$ regiões utilizando a inicialização k-médias . . . . . . . . . . . . . . . . . . . 107

Figura 21 - Curvas de convergência dos parâmetros da imagem São Francisco com $g=3$ regiões e inicialização quantil . . . . . . . . . . . . . . . 108

Figura 22 - Segmentações da imagem São Francisco com $g=3$ regiões utilizando a inicialização quantil . . . . . . . . . . . . . . . . . . . . . 109

Figura 23 - Curvas de convergência dos parâmetros do método SEM com o dicionário de famílias da imagem São Francisco com $g=3$ regiões. . 110

Figura 24 - Curvas de convergência dos parâmetros da imagem Munique completa com $g=3$ e inicialização quantil. . . . . . . . . . . . . . . . . . 112

Figura 25 - Segmentações da imagem Munique completa com $g=3$ regiões utilizando a inicialização quantil . . . . . . . . . . . . . . . . . . . . 113

Figura 26 - Curvas de convergência dos parâmetros da imagem Munique parcial com $g=3$ e inicialização quantil. . . . . . . . . . . . . . . . . . 114

Figura 27 - Segmentações da imagem Munique completa com $g=3$ regiões utilizando a inicialização quantil . . . . . . . . . . . . . . . . . . . 115 


\section{Lista de Tabelas}

Tabela 1 - Descrição das amostras de teste contendo 3 regiões da imagem São Francisco . . . . . . . . . . . . . . . . . . . 68

Tabela 2 - Descrição das amostras de teste contendo 4 regiões da imagem São Francisco . . . . . . . . . . . . . . . . . . . . . 69

Tabela 3 - Descrição das amostras de teste contendo 3 regiões da imagem Munique 70

Tabela 4 - Custo computacional dos métodos de segmentação com a imagem São Francisco contendo 4 regiões . . . . . . . . . . . . . . . . . . . . 72

Tabela 5 - Resultados quantitativos utilizando o método SEM e diferentes inicializações da imagem São Francisco contendo $g=3$ regiões. . . . . . 78

Tabela 6 - Resultados quantitativos da imagem São Francisco com os métodos SEM, EM e k-médias utilizando a inicialização quantil e $g=4$ regiões na iteração de maior log-verossimilhança. . . . . . . . . . . . . . 80

Tabela 7 - Resultados quantitativos da imagem São Francisco com os métodos SEM, EM e k-médias utilizando a inicialização quantil e $g=4$ regiões na iteração $32 \ldots$. . . . . . . . . . . . . . . . . . . . . 8 80

Tabela 8 - Resultados quantitativos da imagem Munique com os métodos SEM, EM e k-médias utilizando a inicialização quantil e $g=3$ regiões na iteração de maior log-verossimilhança. . . . . . . . . . . . . . . . . . 83

Tabela 9 - Resultados quantitativos da imagem Munique com os métodos SEM, EM e k-médias utilizando a inicialização quantil e $g=3$ regiões na iteração 32 . . . . . . . . . . . . . . . . . . . . . . 83

Tabela 10 - Resultados quantitativos utilizando o método SEM do dicionário de famílias e as diferentes inicializações da imagem São Francisco contendo $g=3$ regiões. . . . . . . . . . . . . . . . . . . . . 86

Tabela 11 - Quantidade de seleções das famílias $W, \mathcal{K}_{P}$ e $\mathcal{G}_{P}^{0}$ no método SEM com o modelo do dicionário e famílias da imagem São Francisco. . . . . . 86 


\section{Sumário}

1 Introdução $\quad 15$

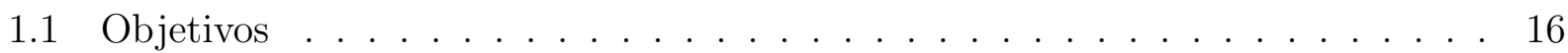

1.2 Contribuições . . . . . . . . . . . . . . . . . . . . . . 17

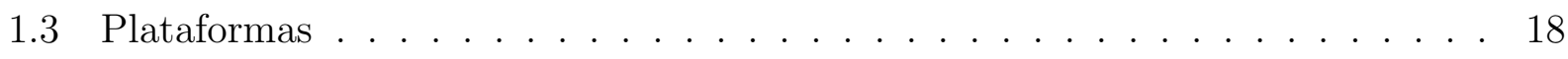

1.4 Colaboração . . . . . . . . . . . . . . . . . . . . 19

1.5 Organização do Texto . . . . . . . . . . . . . . . . . . . . . 19

2 O Algoritmo EM e suas Aplicações $\quad 21$

2.1 O Algoritmo EM . . . . . . . . . . . . . . . . . . . 21

2.2 Aplicação a Modelos de Mistura de Distribuições . . . . . . . . . . . . . . 25

2.3 O Algoritmo SEM . . . . . . . . . . . . . . . . . . 32

2.4 Aplicação à Segmentação de Imagens . . . . . . . . . . . . . . . . . . . . 35

3 Modelos para Dados PolSAR multi-look $\quad 37$

3.1 Imagens SAR . . . . . . . . . . . . . . . . . . 37

3.2 Imagens PolSAR . . . . . . . . . . . . . . . . . . . . . 41

3.3 Modelo Multiplicativo . . . . . . . . . . . . . . . . . . 42

3.4 Modelo para o Ruído Speckle . . . . . . . . . . . . . . . . . . . 43

3.5 Modelos para a Retroespalhamento . . . . . . . . . . . . . . . 44

3.6 Modelo para o Retorno . . . . . . . . . . . . . . . . . 46

3.7 Estimadores para Modelos Polarimétricos . . . . . . . . . . . . . . 50

4 Métodos de Segmentação de Imagens PolSAR multi-look 53

4.1 Trabalhos Relacionados . . . . . . . . . . . . . . . . 54

4.2 Método de Segmentação SEM proposto . . . . . . . . . . . . . . 58 
4.3 Métodos de Inicialização . . . . . . . . . . . . . . . . . . . . . . . . . . . . 64

$\begin{array}{lll}5 & \text { Resultados } & 67\end{array}$

5.1 Descrição das Imagens $\ldots \ldots$. . . . . . . . . . . . . . . . . 67

5.2 Resultados . . . . . . . . . . . . . . . . . . . . 70

6 Conclusão $\quad 87$

6.1 Trabalhos Futuros . . . . . . . . . . . . . . . . . . . . 89

$\begin{array}{ll}\text { Referências } & 91\end{array}$

Apêndice A - Análise Descritiva dos Atributos das Imagens $\quad 99$

A.1 Imagem de São Francisco . . . . . . . . . . . . . . . . . . . . . . . 99

A.2 Imagem Munique . . . . . . . . . . . . . . . . . . . . . . 99

Apêndice B - Resultados da Imagem São Francisco 103

Apêndice C - Resultados da Imagem Munique 111 


\section{Capítulo 1}

\section{Introdução}

Os avanços nas pesquisas voltadas a imagens de Radar de Abertura Sintética (SAR) são de extrema importância ao monitoramento e estudos da superfície terrestre. Os sistemas SAR atuam na faixa de micro-ondas e, por isto, possuem algumas vantagens com relação aos sistemas óticos, como a independência à luz solar e a fatores climáticos e maior penetração na cobertura vegetal da superfície observada.

A desvantagem desse tipo de imagem ocorre com a presença do ruído speckle que é inerente ao seu processo de aquisição e gera um aspecto granuloso na imagem, dificultando a sua interpretação e análise. Devido a esse fato, os métodos clássicos de processamento se tornaram ineficientes a esse tipo de imagem e necessitam ser adaptados. Além disso, muitos métodos estatísticos foram desenvolvidos para imagens SAR (BOMBRUN; BEAULIEU, 2008; CAO et al., 2007; DONG; MILNE; FORSTER, 2001; FRERY; CORREIA; FREITAS, 2007; GAMBINI et al., 2006; TOUZI et al., 2004).

Um problema importante aos dados do tipo SAR é desenvolver modelos estatísticos precisos com o intuito de fornecer técnicas de processamento e análise eficazes. Focalizando nas imagens SAR polarimétricas, este processo de formação dos modelos estatísticos se torna uma tarefa crítica a fim de descrever os dados complexos (possuem informação de fase e amplitude) e as suas propriedades, como o número de looks, a homogeneidade dos alvos e a ocorrência do ruído multiplicativo speckle (FREITAS; FRERY; CORREIA, 2005).

O modelo Multiplicativo é muito utilizado na obtenção de distribuições que ajustam os dados SAR por modelá-los tomando como base a interpretação física dos mesmos (OLIVER; QUEGAN, 1998).

Em Jacobo-Berlles (2005), Freitas, Frery e Correia (2005), há uma revisão bibliográfica sobre as distribuições utilizadas para descrever dados SAR baseadas no modelo Multiplicativo. Focalizando nas imagens polarimétricas multi-look, existem as clássicas distribuições 
Wishart e $\mathcal{K}$ polarimétrica $\left(\mathcal{K}_{P}\right)$ e as distribuições $\mathcal{G}^{0}$ polarimétrica $\left(\mathcal{G}_{P}^{0}\right)$ e $\mathcal{G}$ harmônica. A distribuição Wishart modela os dados polarimétricos multi-look provenientes de regiões homogêneas, como o cultivo. As distribuições $\mathcal{K}_{P}, \mathcal{G}_{P}^{0}$ e $\mathcal{G}^{H}$ possuem um parâmetro de rugosidade para modelar tanto regiões de textura como regiões homogêneas.

A diferença entre as distribuições de textura está na flexibilidade do parâmetro de rugosidade em descrever os diferentes tipos de regiões. A distribuição $\mathcal{K}_{P}$ foi proposta para modelar regiões heterogêneas, como a vegetação. Enquanto que as distribuições $\mathcal{G}_{P}^{0}$ e $\mathcal{G}^{H}$ foram propostas para modelar regiões extremamente heterogêneas, como as áreas urbanas. Em Bombrun e Beaulieu (2008), a distribuição KummerU também foi proposta para descrever regiões de textura.

A clássica distribuição Wishart é muito utilizada no processamento e análise das imagens polarimétricas multi-look (LEE; POTTIER, 2009). Apesar da distribuição Wishart ser robusta à modelagem de dados PolSAR muti-look, o processamento da imagem com este tipo de distribuição define as regiões com características mais homogêneas, podendo ocasionar a mistura entre regiões heterogêneas, como floresta e área urbana, por exemplo. Bombrun e Beaulieu (2008), Freitas, Frery e Correia (2005) e Frery, Correia e Freitas (2007) mostram a importância de se trabalhar com as distribuições adequadas aos tipos de alvos analisados, além de ressaltar a relevância das distribuições de textura na área. Porém, como as distribuições de textura são difíceis de serem tratadas analiticamente, há poucas pesquisas envolvendo estas distribuições.

Na segmentação de imagens ou classificação não supervisionada, alguns exemplos são: os trabalhos de Beaulieu e Touzi (2004) e Yu e Acton (2000) que utilizam a distribuição $\mathcal{K}_{P}$; o trabalho de Bombrun e Beaulieu (2008) utilizando a distribuição KummerU e os trabalhos provenientes desta tese Horta, Mascarenhas e Frery (2008) e Horta et al. (2008) que utilizam a distribuição $\mathcal{G}_{P}^{0}$.

\section{$1.1 \quad$ Objetivos}

Diante das dificuldades encontradas ao utilizar as distribuições de textura no processamento e análise de imagens PolSAR multi-look, esta tese teve o objetivo de estudar a viabilidade de se utilizar os modelos de mistura de distribuições $\mathcal{K}_{P}$ e $\mathcal{G}_{P}^{0}$ como técnicas de agrupamento de dados e, também, comparar a aplicação do modelos de mistura de distribuições Wishart, $\mathcal{K}_{P}$ e $\mathcal{G}_{P}^{0}$ à segmentação de imagens.

Esta tese utilizou o algoritmo SEM combinado com os estimadores obtidos pelo método 
dos momentos para segmentar as imagens SAR polarimétricas multi-look. Analisaram-se os modelos de mistura de uma única família, como os modelos de mistura de distribuições Wishart, $\mathcal{K}_{P}$ ou $\mathcal{G}_{P}^{0}$, e, também, o modelo de mistura do dicionário de famílias contendo estas três distribuições.

\subsection{Contribuições}

As principais contribuições desta tese são:

- Definição de um método de agrupamento de dados aplicado à segmentação de imagens SAR polarimétricas multi-look. Utilizou-se o algoritmo SEM combinado com os estimadores obtidos pelo método dos momentos, tornando o método de segmentação flexível aos diferentes modelos de mistura finita de distribuições: mistura de distribuições Wishart, mistura de distribuições $\mathcal{K}_{p}$ e mistura de distribuições $\mathcal{G}_{P}^{0}$.

- Análises e comparações do método SEM proposto com os três modelos de mistura de uma única família;

- Análises e comparações do método SEM proposto com diferentes inicializações: aleatória, por quantis amostrais e com o método de agrupamento de dados k-médias com medida de dissimilaridade da distância Euclidiana;

- Análise do método de segmentação proposto utilizando um dicionário contendo as três famílias: $\mathcal{D}=\left\{W, \mathcal{K}_{P}, \mathcal{G}_{P}^{0}\right\}$;

- Comparações entre o método SEM proposto e dois métodos da literatura: o algoritmo k-médias com a medida de dissimilaridade obtida pela distribuição Wishart e o algoritmo EM com a mistura de distribuições Wishart (CAO et al., 2007; KERSTEN; LEE; AINSWORTH, 2005). O método k-médias em conjunto com a inicialização por decomposição de alvos é utilizado como método de segmentação na ferramenta educacional PolsARpro (LEE; POTTIER, 2009).

Nesta tese, as pesquisas envolvendo os modelos de mistura de distribuições $\mathcal{G}_{P}^{0}$ ao contexto não supervisionado foram publicadas em Horta, Mascarenhas e Frery (2008) e Horta et al. (2008). Outra aplicação foi na classificação de imagens, na qual as distribuições Wishart e $\mathcal{G}_{P}^{0}$ foram comparadas, que são os textos Horta, Mascarenhas e Frery (2007b) e Horta, Mascarenhas e Frery (2007a).

Este trabalho contou com o apoio financeiro da FAPESP. 


\subsection{Plataformas}

As plataformas utilizadas no desenvolvimento do projeto foram: software comercial de Sensoriamento Remoto ENVI/IDL; o software estatístico R e a ferramenta educacional PolSARpro.

O ENVI e IDL foram utilizados no cálculo das avaliações quantitativas: da porcentagem de acerto total e porcentagem de acerto de cada grupo e do coeficiente Kappa. Utilizou-se o algoritmo k-médias do software ENVI como o método de inicialização por k-médias.

A plataforma $\mathrm{R}$ foi utilizada em todo o desenvolvimento da tese: tanto os algoritmos necessários ao método de segmentação SEM proposto quanto os métodos EM e k-médias com Wishart. Os métodos de inicialização aleatória e por quantis amostrais também foram implementados no R. Como o software R é um ambiente estatístico, todas as rotinas de visualização e manipulação das imagens SAR polarimétricas multi-look também foram implementadas nesta tese.

A ferramenta PolSARpro foi utilizada na obtenção dos dados da imagem São Francisco.

Algumas descrições sobre essas plataformas são:

- O software ENVI/IDL ${ }^{1}$ (Environment for Visualizing Images/Interactive Data Language): O ENVI é um software comercial para processamento de dados de Sensoriamento Remoto, mas que provê a visualização e análise de imagens de virtualmente qualquer tamanho e em inúmeros formatos. Seu fabricante, a RSI (Research Systems Inc), o desenvolveu a partir da linguagem IDL (linguagem flexível de quarta geração com ferramentas de representação gráfica e visualização em forma de funções e rotinas facilmente acessíveis ao usuário). O ENVI é comercializado de duas formas: com, e sem, a linguagem de desenvolvimento IDL. A versão com o IDL permite integrar ao ENVI aplicações que foram desenvolvidas no IDL.

- O software estatístico $\mathrm{R}^{2}$ : É um ambiente de trabalho que fornece uma enorme variedade de análises estatísticas dos dados. Possui tanto funções e pacotes estatísticos quando recursos gráficos para visualização. Os processamentos podem ser executadas por linha de comando ou através de programas implementados no próprio R. É uma versão gratuita do ambiente estatístico S-PLUS, desenvolvido pelo Laboratório Bell da Lucent Technologies.

- A ferramenta PolSARpro ${ }^{3}$ (Polarimetric SAR Data Processing and Educational Tool):

\footnotetext{
${ }^{1}$ Disponível em http://www.rsinc.com.

${ }^{2}$ Disponível em http://www.r-project.org/.

${ }^{3}$ Disponível em http://earth.esa.int/polsarpro/.
} 
software educacional que provê a visualização, análise e processamento de imagens SAR polarimétricas de tamanho e formatos variados. Todo o desenvolvimento do projeto PolSARpro, tanto software (inclusive código fonte) quanto tutoriais e imagens, são distribuídos pela Agência Espacial Européia (ESA - European Space Agency).

\subsection{Colaboração}

Esta tese contou com a colaboração do Prof. Dr. Alejandro César Frery Orgambide, professor titular do Instituto de Computação da Universidade Federal de Alagoas (UFAL). No primeiro semestre de 2007, no início das pesquisas sobre as imagens PolSAR multi-look, houve um estágio de seis meses com a supervisão do Prof. Dr. Alejandro C. Frery no Instituto de Computação da UFAL. Além disso, o Prof. Dr. Alejandro C. Frery continuou com as colaborações em todas as publicações desta tese.

\subsection{Organização do Texto}

A descrição dessa tese está estruturada nos seguintes capítulos:

- Capítulo 2: Principais conceitos sobre o algoritmo EM, sobre o modelo de mistura de distribuições e quando este é utilizado com o EM, sobre a aplicação da mistura de distribuições à segmentação de imagens;

- Capítulo 3: Modelos polarimétricos clássicos Wishart e $\mathcal{K}_{P}$ e modelo $\mathcal{G}_{P}^{0}$, assim como os estimadores de cada modelo que foram utilizados no método de segmentação SEM. Além disso, há alguns conceitos introdutórios sobre as imagens SAR polarimétricas;

- Capítulo 4: Métodos de segmentações aplicados a imagens PolSAR multi-look, incluindo o método de segmentação SEM proposto;

- Capítulo 5: Resultados;

- Capítulo 6: Conclusões e trabalhos futuros. 


\section{Capítulo 2}

\section{O Algoritmo EM e suas Aplicações}

Este capítulo apresenta a revisão bibliográfica de alguns conceitos introdutórios que compõem esta tese, são eles: o algoritmo EM, sua aplicação a modelos de mistura de distribuições, o algoritmo SEM (versão estocástica do EM) e a aplicação de todo este contexto à segmentação de imagens. Os estudos sobre os dois primeiros assuntos são extensos e variados. A intensidade da pesquisa na área pode ser avaliada pelo fato de o livro de McLachlan e Peel (2000) citar mais de oitocentas referências. Em McLachlan e Peel (2000) há um vasto estudo sobre a mistura de distribuições, como a utilização do algoritmo EM na estimação dos parâmetros da mistura. Este capítulo fornece um resumo direcionado sobre esses assuntos em seções que se inter-relacionam.

\section{$2.1 \quad \mathrm{O}$ Algoritmo EM}

O algoritmo EM (Expectation-Maximization) é uma solução geral a diversos problemas de estimação por máxima verossimilhança no contexto de dados incompletos. Qualquer problema de estimação que se adeque a este contexto pode ser resolvido pelo EM; por exemplo, na análise de agrupamento de dados (clustering), o grupo é a informação a ser preenchida nos dados incompletos (DUDA; HART; STORK, 2001). Alguns textos que tratam detalhadamente este assunto e exemplificam a vasta aplicabilidade do algoritmo EM são os de Dempster, Laird e Rubin (1977), de McLachlan e Krishnan (1997), de McLachlan e Peel (2000) e de Redner e Walker (1984).

A definição do algoritmo EM é apresentada na próxima seção. 


\subsubsection{Definição}

O algoritmo EM é um método iterativo de estimação de parâmetros através do conceito de dados incompletos. Os conceitos fundamentais deste algoritmo foram introduzidos por Dempster, Laird e Rubin (1977). Nesse trabalho, os autores estruturaram os resultados (teóricos e aplicados) até então disponíveis, propuseram uma versão geral da técnica, demonstraram as provas das principais propriedades e definiram o nome EM ao algoritmo. Um exemplo básico sobre o conceito de dados incompletos é fornecido a seguir.

Considere a variável aleatória $U \sim \mathcal{D}(\boldsymbol{\theta}), \boldsymbol{\theta} \subset \mathbb{R}^{P}$, multivariada em $\mathbb{R}^{3}$. Observamos $\boldsymbol{u}=\left(u_{1}, \ldots, u_{10}\right)$ incompleto:

$$
\boldsymbol{u}=\left(\begin{array}{cccccccccc}
u_{1,1} & u_{1,2} & u_{1,3} & u_{1,4} & u_{1,5} & u_{1,6} & u_{1,7} & ? & ? & ? \\
u_{2,1} & u_{2,2} & u_{2,3} & u_{2,4} & u_{2,5} & ? & ? & u_{2,8} & u_{2,9} & u_{2,10} \\
u_{3,1} & u_{3,2} & u_{3,3} & u_{3,4} & u_{3,5} & ? & ? & u_{3,8} & u_{3,9} & u_{3,10}
\end{array}\right)
$$

em que "?" representa os dados que não foram observados, chamados de dados faltantes. Analisando o conjunto $\boldsymbol{u}$ como uma partição $\boldsymbol{u}=\left(\boldsymbol{u}_{\text {obs }}, \boldsymbol{u}_{\text {falt }}\right)$, tem-se os subconjuntos $\boldsymbol{u}_{\text {obs }}$ contendo as informações observadas e $\boldsymbol{u}_{\text {falt }}$ representando o que não foi observado (parte incompleta).

Como existem alguns dados não observados $\left(\boldsymbol{u}_{\text {falt }}\right)$, não se pode estimar $\boldsymbol{\theta}$ por máxima verossimilhança diretamente, tornando o problema um caso de preenchimento de informações dos dados que faltam para se estimar os parâmetros.

Esta é a ideia básica do algoritmo EM que soluciona este problema de forma iterativa e através de dois passos:

- Passo-E - Computa a esperança da log-verossimilhança reduzida condicionada ao dado observado e às estimativas do parâmetro atual, com o intuito de preencher as informações que faltam. No caso, a iteração tem numeração $(k+1)$ e o parâmetro corrente é $\boldsymbol{\theta}^{(k)}$, com $k \geq 0$. A esperança condicional da log-verossimilhança reduzida é definida como

$$
Q\left(\boldsymbol{\theta} ; \boldsymbol{\theta}^{(k)}\right)=\mathbb{E}_{\boldsymbol{\theta}^{(k)}}\left\{\ell_{c}^{*}(\boldsymbol{\theta}) \mid \boldsymbol{u}_{o b s}\right\}
$$

na qual

$$
\ell_{c}^{*}(\boldsymbol{\theta})=\log (L(\boldsymbol{\theta}))=\log \left(f\left(u_{1}, \ldots, u_{n} \mid \boldsymbol{\theta}\right)\right),
$$

desprezando os termos que não dependem do parâmetro.

Neste caso, $L(\boldsymbol{\theta})=f\left(u_{1}, \ldots, u_{n} \mid \boldsymbol{\theta}\right)$ é a função de verossimilhança do parâmetro $\boldsymbol{\theta}$ com base na observação da amostra $\boldsymbol{u}=\left(u_{1}, \ldots, u_{n}\right)$. Quando se tem um conjunto 
$U_{1}, \ldots, U_{n}$ de variáveis aleatórias independentes e identicamente distribuídas, a distribuição conjunta é o produto de densidades de probabilidade iguais e, com isso, a verossimilhança é definida por $L(\boldsymbol{\theta})=\prod_{i=1}^{n} f\left(u_{i} \mid \boldsymbol{\theta}\right)$.

Para facilitar os cálculos, e aproveitando que se trata de funções positivas, podese utilizar o logaritmo da função de verossimilhança $\ell(\boldsymbol{\theta})=\log L(\boldsymbol{\theta})$, chamado de "função de log-verossimilhança", e facilitando ainda mais, pode-se descartar todos os termos de $\ell$ que não dependem do parâmetro $\boldsymbol{\theta}$, trabalhando-se com a função de log-verossimilhança reduzida $\ell^{*}(\boldsymbol{\theta})$.

- Passo-M - Atualiza os parâmetros estimados de acordo com a maximização da esperança condicional $Q\left(\boldsymbol{\theta} ; \boldsymbol{\theta}^{(k)}\right)$, dadas as informações completas. A escolha de $\boldsymbol{\theta}^{(k+1)}$ pode ser ampliada a qualquer valor de $\boldsymbol{\theta} \in \Theta$ que maximize $Q\left(\boldsymbol{\theta} ; \boldsymbol{\theta}^{(k)}\right)$, ou seja,

$$
Q\left(\boldsymbol{\theta}^{(k+1)} ; \boldsymbol{\theta}^{(k)}\right) \geq Q\left(\boldsymbol{\theta} ; \boldsymbol{\theta}^{(k)}\right),
$$

para todo $\boldsymbol{\theta} \in \Theta$, sendo $\Theta$ é o espaço paramétrico.

Uma alternativa é o conjunto de pontos definidos por

$$
\widehat{\boldsymbol{\theta}}^{(k+1)}=\arg \max _{\boldsymbol{\theta} \in \boldsymbol{\Theta}} Q\left(\boldsymbol{\theta} ; \boldsymbol{\theta}^{(k)}\right),
$$

cuja resolução pode ser obtida por $\nabla Q\left(\boldsymbol{\theta} ; \boldsymbol{\theta}^{(k)}\right)=\mathbf{0}$.

A cada iteração é necessário verificar a convergência do algoritmo EM, ou seja, se $L\left(\boldsymbol{\theta}^{(k+1)}\right) \geq L\left(\boldsymbol{\theta}^{(k)}\right)$. Há diversas formas de aferir a convergência do algoritmo EM. Uma delas utiliza critérios baseados no erro absoluto relativo entre passos sucessivos dos estimadores ou da verossimilhança, são eles:

$$
\left|\frac{L\left(\boldsymbol{\theta}^{(k)}\right)-L\left(\boldsymbol{\theta}^{(k+1)}\right)}{L\left(\boldsymbol{\theta}^{(k+1)}\right)}\right|<\epsilon
$$

$\mathrm{ou}$

$$
\max \left\{\left|\frac{\widehat{\boldsymbol{\theta}}^{(k)}-\widehat{\boldsymbol{\theta}}^{(k+1)}}{\widehat{\boldsymbol{\theta}}^{(k+1)}}\right|\right\}<\epsilon .
$$

Se o erro absoluto relativo for menor do que um certo limiar estipulado previamente (representado por $\epsilon$ ), então, considera-se que o algoritmo convergiu e a execução do algoritmo é finalizada. Caso contrário, os passos E e M são repetidos alternadamente.

O algoritmo EM é largamente utilizado por ser fácil de ser implementado. Contudo, em algumas situações, apresenta problemas na convergência do método. A próxima seção comenta sobre a convergência do algoritmo EM e cita algumas extensões. 


\subsubsection{Convergência e Extensões}

Como comentado na seção anterior, o EM é um algoritmo iterativo e simples que aproxima a maximização da função de verossimilhança. A desvantagem do EM está relacionada à susceptibilidade do método ao contexto aplicado. Em muitas situações, os problemas relacionados ao EM ocorrem na convergência do método (MCLACHLAN; KRISHNAN, 1997; WU, 1983).

No caso do ajuste a modelos de mistura de distribuições finita, a convergência do EM é afetada sensivelmente pelos tipos de distribuição e inicialização utilizados. Este fato pode resultar na convergência do método para um máximo local ao invés do máximo global ou num método com alto custo computacional por necessitar de muitas iterações até a sua convergência (MCLACHLAN; PEEL, 2000). O algoritmo também pode convergir para as fronteiras do espaço paramétrico, principalmente, quando a mistura contém um grande número de distribuições (FIGUEIREDO; JAIN, 2002).

Além disso, a maximização da esperança condicional $Q\left(\boldsymbol{\theta} ; \boldsymbol{\theta}^{(k)}\right)$ obterá solução em forma fechada de acordo com as distribuições utilizadas na mistura. Moser, Zerubia e Serpico (2006) citam algumas distribuições que não têm solução analítica em forma fechada para este tipo de problema, cujos exemplos são as distribuições $\mathcal{K}$ e Weibull utilizadas nos modelos de mistura para dados SAR.

Como o passo-M do EM é restrito a soluções em forma fechada, seus próprios autores Dempster, Laird e Rubin (1977) propuseram uma versão mais geral ao método: o algoritmo GEM (Generalized EM). O GEM é um algoritmo flexível, podendo ser aplicado aos exemplos em que não há solução em forma fechada. No caso do algoritmo GEM, a esperança condicional $Q\left(\boldsymbol{\theta} ; \boldsymbol{\theta}^{(k)}\right)$ é maximizada sobre as estimativas atuais $\left(\boldsymbol{\theta}=\boldsymbol{\theta}^{(k)}\right)$, enquanto que no EM a maximização é realizada sobre todos os valores do espaço paramétrico $(\boldsymbol{\theta} \in \Theta)$.

Muitas extensões foram propostas em busca de uma possível melhora no desempenho do método EM. Algumas destas versões são encontradas nos textos de McLachlan e Krishnan (1997) e McLachlan e Peel (2000).

Há versões que foram propostas para melhorar a aplicação do EM ao contexto de mistura de distribuições. Alguns exemplos são os algoritmos CEM, CEM ${ }^{2}$ e SEM. O algoritmo CEM (Classification EM) acrescenta um passo de classificação de dados ao algoritmo EM (BIERNACKI; CELEUX; GOVAERT, 2003). O algoritmo CEM² (Component-Wise EM) fornece uma versão sequencial na atualização dos parâmetros do modelo de mistura no passo-M do EM (FIGUEIREDO; JAIN, 2002). O algoritmo SEM (Stochastic EM) acrescenta um passo de amostragem estocástica ao algoritmo EM (BOUGUILA; ZIOU, 2006; 
DELIGNON; MARZOUKI; PIECZYNSKI, 1997; MOSER; ZERUBIA; SERPICO, 2006).

Nesta tese, utilizou-se o algoritmo SEM para segmentar as imagens polarimétricas multi-look. A inclusão da amostragem estocástica ao algoritmo EM torna o SEM um algoritmo mais flexível aos modelos de mistura com distribuições difíceis de serem tratadas analiticamente, como é o caso das distribuições $\mathcal{K}$ e $\mathcal{G}^{0}$ polarimétricas utilizadas nesta tese. Segundo Moser, Zerubia e Serpico (2006), o passo-M do SEM pode ser substituído por estimadores obtidos por outros métodos, como o método dos momentos. A Seção 2.3 discute o método SEM, enquanto a próxima seção apresenta a aplicação do EM a modelos de mistura de distribuições.

\subsection{Aplicação a Modelos de Mistura de Distribuições}

Esta seção apresenta a revisão de alguns conceitos sobre os modelos de mistura de distribuições e a utilização deste contexto como uma técnica de agrupamento de dados (DUDA; HART; STORK, 2001). Os conceitos básicos sobre o algoritmo EM e dados incompletos foram discutidos na seção anterior.

A próxima seção inicia a discussão sobre os modelos de mistura de distribuições.

\subsubsection{Modelos de Mistura de Distribuições}

Os modelos de mistura de distribuições são métodos flexíveis de modelar funções de distribuições de probabilidades complexas a partir de elementos mais simples e tratáveis. Esses modelos podem ser aplicados a uma variedade de fenômenos estocásticos. Várias pesquisas e aplicações foram realizadas sobre este assunto, como discutidas nos livros de Everitt e Hand (1981), McLachlan e Peel (2000) e Titterington, Smith e Makov (1985). O artigo de Redner e Walker (1984) também é uma importante revisão bibliográfica sobre a mistura de distribuições, abordando as técnicas de estimação de parâmetros, em especial, o algoritmo EM. Nesta seção, os assuntos abordados se baseiam nestes livros e artigo, entre outros artigos citados no decorrer das explanações.

Os modelos de mistura de distribuições foram empregados com sucesso em diversas áreas (MCLACHLAN; PEEL, 2000). Por exemplo, no reconhecimento de padrões e visão 
computacional, algumas pesquisas foram propostas por Bouguila e Ziou (2006), Kersten, Lee e Ainsworth (2005), Khan, Yang e Zhang (2007), Moser, Zerubia e Serpico (2006) e Yang e Liu (2002). Na segmentação de imagens, em particular, algumas pesquisas são as de Alfò, Nieddu e Vicari (2008), Delignon, Marzouki e Pieczynski (1997), Derrode e Pieczynski (2004), Mignotte et al. (2000)e Yang e Krishnan (2004).

Os conceitos e problemas associados aos modelos de mistura de distribuições são apresentados na próxima seção.

\subsubsection{Definição}

Os modelos de mistura são famílias de distribuições formadas pela composição estocástica de mais de uma distribuição básica. Estes modelos permitem descrever o comportamento de uma variável aleatória como sendo controlado pela distribuição $D_{1}$ com probabilidade $\rho_{1}$, pela distribuição $D_{2}$ com probabilidade $\rho_{2}$, etc. A densidade dessa variável aleatória é a combinação convexa das densidades que caracterizam essas distribuições, sendo as respectivas probabilidades os fatores da combinação.

Em outras palavras, um modelo de mistura para a variável aleatória $U$ é caracterizado pelas distribuições $D_{1}, D_{2}, \ldots$, cada uma com densidade $f_{1}, f_{2}, \ldots$ e pelas probabilidades $\rho_{1}, \rho_{2}, \ldots$ Em geral, as misturas são finitas, ou seja, uma população com variável aleatória $U$ é definida por $g$ distribuições $D_{1}, \ldots, D_{g}$ e probabilidades $\rho_{1}, \ldots, \rho_{g}$, em que $\rho_{j} \geqslant 0$ e $\sum_{j=1}^{g} \rho_{j}=1$.

A variável aleatória $U$ de uma mistura finita possui função densidade de probabilidade

$$
f(u)=\sum_{j=1}^{g} \rho_{j} f_{j}\left(u \mid \boldsymbol{\theta}_{j}\right),
$$

na qual cada distribuição $D_{j}$ é caracterizada por um vetor de parâmetros $\boldsymbol{\theta}_{j}$. Esta situação é denotada por $U \sim M(\boldsymbol{\theta}, \boldsymbol{\rho})$, sendo $\boldsymbol{\theta}=\left(\boldsymbol{\theta}_{1}, \ldots, \boldsymbol{\theta}_{g}\right)$ e $\boldsymbol{\rho}=\left(\rho_{1}, \ldots, \rho_{g}\right)$. As probabilidades $\rho_{1}, \ldots, \rho_{g}$ são conhecidas como proporções da mistura, as densidades $f_{1}, \ldots, f_{g}$ são as componentes de densidade e o $g$ é o número de distribuições que formam a mistura finita (EVERITT; HAND, 1981; MCLACHLAN; PEEL, 2000).

Dois tipos de parâmetros devem ser estimados na mistura: os valores das proporções contidas em $\boldsymbol{\rho}$ e os parâmetros $\boldsymbol{\theta}$ que caracterizam as distribuições (ou suas respectivas componentes). Quando os estimadores $(\widehat{\boldsymbol{\rho}}, \widehat{\boldsymbol{\theta}})$ são obtidos, pode-se analisar o conjunto de dados estudado, sendo os dados considerados ocorrências de variáveis aleatórias $U_{1}, \ldots, U_{n}$ 
e $n$ o tamanho da amostra. Em geral, o número de distribuições da mistura $g$ é conhecido.

A seguir serão expostos alguns problemas envolvendo o modelo de mistura de distribuições.

\subsubsection{Problemas}

Alguns dos problemas decorrentes do uso da classe de modelos de mistura de distribuições são:

1. A escolha das distribuições $D_{1}, \ldots, D_{g}$ a serem empregadas para compor a distribuição $M$ que descreve a mistura;

2. A procura de técnicas de estimação dos parâmetros da distribuição da mistura, ou seja, estimar os parâmetros $(\boldsymbol{\rho}, \boldsymbol{\theta})$;

3. A determinação prática de tamanhos de amostras para estimar os parâmetros $(\boldsymbol{\rho}, \boldsymbol{\theta})$.

4. A decisão de utilizar $g$ conhecido ou não e, caso se opte por incluí-lo no conjunto paramétrico desconhecido, escolher a técnica mais adequada para estimálo (MCLACHLAN; PEEL, 2000).

Quando soluções a estes quatro problemas são propostas, pode-se aplicar os modelos de mistura de distribuições aos dados analisados. No que se segue, sempre consideraremos $g$ conhecido a priori.

\subsubsection{Estimação por Máxima Verossimilhança usando o EM}

Considerando o modelo de mistura de distribuições $M(\boldsymbol{\theta}, \boldsymbol{\rho})$ e seus problemas, definidos na Seção 2.2.1, uma forma usual de se estimar os parâmetros $(\boldsymbol{\theta}, \boldsymbol{\rho})$ é através da técnica de máxima verossimilhança (DUDA; HART; STORK, 2001; EVERITT; HAND, 1981; MCLACHLAN; PEEL, 2000). Supõe-se que o número de distribuições $g$ é conhecido e os parâmetros a serem estimados são $\boldsymbol{\psi}=(\boldsymbol{\theta}, \boldsymbol{\rho})$. Considerou-se, nesta tese, a notação $\boldsymbol{\psi}$ para o conjunto paramétrico da mistura de distribuições finita. 
Considerando que a variável aleatória contínua $U \sim M(\boldsymbol{\theta}, \boldsymbol{\rho})$ possui a função de densidade de probabilidade $f(u \mid \boldsymbol{\psi})$ definida na Equação (2.8) (página 26), então um estimador de máxima verossimilhança de $\boldsymbol{\psi}$, se existir, é qualquer $\boldsymbol{\psi} \in \boldsymbol{\Psi}$ satisfazendo

$$
\widehat{\boldsymbol{\psi}}=\arg \max _{\boldsymbol{\psi} \in \boldsymbol{\Psi}} L(\boldsymbol{\psi})
$$

sendo $L(\boldsymbol{\psi})=f\left(u_{1}, \ldots, u_{n} \mid \boldsymbol{\psi}\right)$ é a função de verossimilhança e $\boldsymbol{\Psi}$ o espaço paramétrico.

Supõe-se que $\boldsymbol{u}=\left(u_{1}, \ldots, u_{n}\right)$ são ocorrências independentes de $U$. Nestas condições, a função de verossimilhança é

$$
L(\boldsymbol{\psi})=\prod_{i=1}^{n} f\left(u_{i} \mid \boldsymbol{\psi}\right)=\prod_{i=1}^{n} \sum_{j=1}^{g} \rho_{j} f_{j}\left(u_{i} \mid \boldsymbol{\theta}_{j}\right),
$$

e a log-verossimilhança reduzida $\ell^{*}(\boldsymbol{\psi})$ é definida descartando todos os termos da logverossimilhança que não dependam dos parâmetros sendo estimados

$$
\ell(\boldsymbol{\psi})=\sum_{i=1}^{n} \log \left(\sum_{j=1}^{g} \rho_{j} f_{j}\left(u_{i} \mid \boldsymbol{\theta}_{j}\right)\right),
$$

em que $\boldsymbol{\psi}=\left(\rho_{1}, \ldots, \rho_{g-1}, \boldsymbol{\theta}^{T}\right)^{T}$ e $\rho_{g}=1-\sum_{j=1}^{g-1} \rho_{j}$.

Sempre que possível, a função de log-verossimilhança reduzida é maximizada pela resolução de um sistema de equações $\nabla \ell^{*}(\boldsymbol{\psi})=\mathbf{0}$. Frequentemente, quando esta aborgadem é aplicada a modelos de mistura de distribuições, ela não possui solução analítica explícita.

Muitas pesquisas foram realizadas para resolver este problema (MCLACHLAN; KRISHNAN, 1997). Alguns trabalhos são os de Day (1969), Hasselblad (1966, 1969) e Wolfe (1965, 1967, 1970) que simplificaram os cálculos realizados com a estimação de máxima verossimilhança em modelos de mistura com distribuições específicas, encontrando alternativas com a manipulação do sistema de equações, cujos resultados são as Equações (2.12) e (2.13). Nessas pesquisas, foi observado também que, em casos especiais, as manipulações sugerem uma computação iterativa para solucionar o sistema de equações. Após o artigo de Dempster, Laird e Rubin (1977), foi verificado que esse processo iterativo é uma aplicação direta do algoritmo EM, cujas discussões podem ser encontradas em McLachlan e Peel (2000).

Diante das manipulações feitas no sistema de equações $\nabla \ell^{*}(\boldsymbol{\psi})=\mathbf{0}$, a estimação de máxima verossimilhança de $\boldsymbol{\psi}$ passou a satisfazer as equações

$$
\widehat{\rho}_{j}=\frac{\sum_{i=1}^{n} \tau_{j}\left(\widehat{\psi} \mid u_{i}\right)}{n}, j=1, \ldots, g
$$


e

$$
\sum_{j=1}^{g} \sum_{i=1}^{n} \tau_{j}\left(\widehat{\boldsymbol{\psi}} \mid u_{i}\right) \frac{\partial \log f_{j}\left(u_{i} \mid \widehat{\boldsymbol{\theta}}_{j}\right)}{\partial \boldsymbol{\theta}_{j}}=\mathbf{0}
$$

nas quais

$$
\tau_{j}\left(\widehat{\boldsymbol{\psi}} \mid u_{i}\right)=\frac{\widehat{\rho}_{j} f_{j}\left(u_{i} \mid \widehat{\boldsymbol{\theta}}_{j}\right)}{f\left(u_{i} \mid \widehat{\boldsymbol{\psi}}\right)}, j=1, \ldots, g
$$

é a probabilidade a posteriori de $u_{i}$ pertencer a componente $f_{j}$ da mistura (MCLACHLAN; PEEL, 2000).

Com as simplificações resultantes nas Equações (2.12) e (2.13), Orchard e Woodbury (1972) formularam um exemplo mais geral para calcular iterativamente o estimador de máxima verossimilhança utilizando conceitos iniciais de dados incompletos. Butler (1986) destacou alguns exemplos iniciais de pesquisas nesta área por Jeffreys (1932) e Newcomb (1886).

Em 1977, Dempster, Laird e Rubin introduziram o algoritmo iterativo ExpectationMaximization (EM) (DEMPSTER; LAIRD; RUBIN, 1977). Este método é uma solução geral a diversos problemas de estimação de parâmetros com dados incompletos, ou aplicáveis a esse contexto, como é o caso da mistura de distribuições. As definições básicas sobre o algoritmo EM foram discutidas na Seção 2.1 (página 21).

Quando o método EM é aplicado à mistura de distribuições, tem-se um conjunto de dados completo $\boldsymbol{u}_{c}=(\boldsymbol{u}, \boldsymbol{r})$ composto por um conjunto $\boldsymbol{u}$ de valores observados e um conjunto $\boldsymbol{r}$ de valores não observados. O conjunto observado $\boldsymbol{u}=\left(u_{1}, \ldots, u_{n}\right)$ é modelado por uma mistura de distribuições $M(\boldsymbol{\theta}, \boldsymbol{\rho})$ definida na Equação (2.8) (página 26). A parte não observada é composta por $n$ rótulos $\boldsymbol{r}=\left(\boldsymbol{r}_{1}, \ldots, \boldsymbol{r}_{n}\right)$ associados a cada observação $\left(u_{1}, \ldots, u_{n}\right)$, indicando qual componente descreverá cada observação. Os rótulos são vetores binários $\boldsymbol{r}_{i}=\left(r_{1 i}, \ldots, r_{g i}\right)$, com

$$
r_{j i}= \begin{cases}1 & \text { se } u_{i} \in D_{j}, \\ 0 & \text { caso contrário; }\end{cases}
$$

em que a $j$-ésima componente corresponde à distribuição $D_{j}$ (MCLACHLAN; PEEL, 2000).

Iremos supor que $\left(\boldsymbol{r}_{1}, \ldots, \boldsymbol{r}_{n}\right)$ são ocorrências de variáveis aleatórias independentes e identicamente distribuídas seguindo uma distribuição multinomial em $g$ categorias com probabilidades $\left(\rho_{1}, \ldots, \rho_{g}\right)$, definida por $R_{1}, \ldots, R_{n} \sim \mathcal{M}_{g}(1, \boldsymbol{\rho})$ com função de probabilidade

$$
f\left(\boldsymbol{r}_{i} \mid \boldsymbol{\rho}\right)=\frac{1 !}{\prod_{j=1}^{g} r_{j i} !} \prod_{j=1}^{g} \rho_{j}^{r_{j i}}
$$


cujo logaritmo é

$$
\log f\left(\boldsymbol{r}_{i} \mid \boldsymbol{\rho}\right)=\sum_{j=1}^{g} r_{j i} \log \rho_{j}
$$

para $i=1, \ldots, n$. Enquanto $\left(u_{1}, \ldots, u_{n}\right)$ dado $\left(\boldsymbol{r}_{1}, \ldots, \boldsymbol{r}_{n}\right)$, respectivamente, são independentes condicionalmente com o logaritmo da função densidade de probabilidade

$$
\log f\left(u_{i} \mid \boldsymbol{r}_{i}, \boldsymbol{\theta}\right)=\sum_{j=1}^{g} r_{j i} \log f_{j}\left(u_{i} \mid \boldsymbol{\theta}_{j}\right)
$$

para $i=1, \ldots, n$.

Pelo teorema de Bayes, o logaritmo da função densidade de probabilidade do dado completo foi simplificado por

$$
\log f\left(\boldsymbol{u}_{c} \mid \boldsymbol{\psi}\right)=\sum_{i=1}^{n}\left\{\log f\left(\boldsymbol{r}_{i} \mid \boldsymbol{\rho}\right)+\log f\left(u_{i} \mid \boldsymbol{r}_{i}, \boldsymbol{\theta}\right)\right\}
$$

e resulta na log-verossimilhança dos parâmetros $\boldsymbol{\psi}$

$$
\ell_{c}(\boldsymbol{\psi})=\sum_{j=1}^{g} \sum_{i=1}^{n} r_{j i}\left\{\log \rho_{j}+\log f_{j}\left(u_{i} \mid \boldsymbol{\theta}_{j}\right)\right\} .
$$

No algoritmo EM, os parâmetros são estimados alternando dois passos: o Passo-E (Expectation) que calcula a esperança da log-verossimilhança $\left(\ell_{c}(\boldsymbol{\psi})\right)$, condicionada ao dado observado e valores iniciais definidos aos parâmetros $\boldsymbol{\psi}$; o Passo-M (Maximization) que maximiza a log-verossimilhança para estimar os parâmetros em função dos resultados obtidos no Passo-E.

\subsubsection{Passo-E (Expectation)}

$\mathrm{Na}(k+1)$-ésima iteração, o Passo-E calcula a esperança condicional dadas as observações $\boldsymbol{u}$ e os parâmetros correntes $\boldsymbol{\psi}^{(k)}$, cuja equação é definida por

$$
Q\left(\boldsymbol{\psi} ; \boldsymbol{\psi}^{(k)}\right)=\mathbb{E}_{\boldsymbol{\psi}^{(k)}}\left\{\ell_{c}^{*}(\boldsymbol{\psi}) \mid \boldsymbol{u}\right\}
$$

na qual $\ell_{c}^{*}(\boldsymbol{\psi})$ é a $\log$-verossimilhança reduzida de $\ell_{c}(\boldsymbol{\psi})$ (MCLACHLAN; PEEL, 2000). 
A função da Equação (2.20) é linear em relação aos dados não observados $\boldsymbol{r}$ :

$$
\begin{aligned}
Q\left(\boldsymbol{\psi} ; \boldsymbol{\psi}^{(k)}\right) & =\mathbb{E}_{\boldsymbol{\psi}^{(k)}}\left\{\sum_{j=1}^{g} \sum_{i=1}^{n} R_{j i}\left\{\log \rho_{j}+\log f_{j}\left(u_{i} \mid \boldsymbol{\theta}_{j}\right)\right\} \mid \boldsymbol{u}\right\} \\
& =\sum_{j=1}^{g} \sum_{i=1}^{n} \mathbb{E}_{\boldsymbol{\psi}^{(k)}}\left[R_{j i} \mid \boldsymbol{u}\right]\left\{\log \rho_{j}+\log f_{j}\left(\boldsymbol{r}_{i} \mid \boldsymbol{\theta}_{j}\right)\right\}
\end{aligned}
$$

Então, o Passo-E se resume a calcular a esperança condicional da variável aleatória $R_{j i}$ dado $u_{i}$, ou seja,

$$
\mathbb{E}_{\boldsymbol{\psi}^{(k)}}\left[R_{j i} \mid u_{i}\right]=\operatorname{pr}\left(R_{j i}=1 \mid U_{i}=u_{i} ; \boldsymbol{\psi}^{(k)}\right)=\tau_{j}\left(\boldsymbol{\psi}^{(k)} \mid u_{i}\right)
$$

em que

$$
\tau_{j}\left(\boldsymbol{\psi}^{(k)} \mid u_{i}\right)=\frac{\rho_{j}^{(k)} f_{j}\left(u_{i} \mid \boldsymbol{\theta}_{j}^{(k)}\right)}{f\left(u_{i} \mid \boldsymbol{\psi}^{(k)}\right)},
$$

é a probabilidade a posteriori de cada $j=1, \ldots, g$ (ver Equação (2.14) na página 29). A esperança condicional da log-verossimilhança reduzida resulta em (MCLACHLAN; PEEL, 2000):

$$
Q\left(\boldsymbol{\psi} ; \boldsymbol{\psi}^{(k)}\right)=\mathbb{E}_{\boldsymbol{\psi}^{(k)}}\left\{\ell_{c}^{*}\left(\boldsymbol{\psi}^{(k)}\right) \mid \boldsymbol{u}\right\}=\sum_{j=1}^{g} \sum_{i=1}^{n} \tau_{j}\left(\boldsymbol{\psi}^{(k)} \mid u_{i}\right)\left\{\log \rho_{j}+\log f_{j}\left(u_{i} \mid \boldsymbol{\theta}_{j}\right)\right\} .
$$

\subsubsection{Passo-M (Maximization)}

$\mathrm{Na}(k+1)$-ésima iteração, o Passo-M calcula as estimativas dos parâmetros. Valores são atualizados em função dos estimadores

$$
\widehat{\boldsymbol{\psi}}^{(k+1)}=\arg \max _{\boldsymbol{\psi}^{(k+1)} \in \boldsymbol{\Psi}} Q\left(\boldsymbol{\psi} ; \boldsymbol{\psi}^{(k)}\right)
$$

No caso dos modelos de mistura de distribuições, o cálculo das proporções $\boldsymbol{\rho}^{(k+1)}$ não depende dos parâmetros $\boldsymbol{\theta}^{(k+1)}$ das componentes, como descrito na Equação (2.12) (página 28), cuja atualização é definida por

$$
\widehat{\rho}_{j}^{(k+1)}=\frac{\sum_{i=1}^{n} \tau_{j}\left(\boldsymbol{\psi}^{(k)} \mid u_{i}\right)}{n}, j=1, \ldots, g .
$$

Os parâmetros $\boldsymbol{\theta}^{(k+1)}$ são obtidos com a resolução do sistema de equações (MCLACH- 
LAN; PEEL, 2000)

$$
\sum_{j=1}^{g} \sum_{i=1}^{n} \tau_{j}\left(\boldsymbol{\psi}^{(k)} \mid u_{i}\right) \frac{\partial \log f_{j}\left(u_{i} \mid \boldsymbol{\theta}_{j}\right)}{\partial \boldsymbol{\theta}}=\mathbf{0}
$$

Frequentemente, as distribuições $D_{i}$ pertencem à mesma família, como os modelos de mistura de distribuições gaussianas, simplificando a resolução analítica desse cálculo. Por exemplo, na mistura de distribuições gaussianas multivariadas, os parâmetros são $\boldsymbol{\theta}_{j}=\left(\boldsymbol{\mu}_{j}, \Sigma_{j}\right)$, sendo $\boldsymbol{\mu}_{j}$ o vetor média e $\Sigma_{j}$ a matriz de covariância da $j$-ésima componente. As atualizações são

$$
\widehat{\boldsymbol{\mu}}_{j}^{(k+1)}=\frac{\sum_{i=1}^{n} u_{i} \tau_{j}\left(\boldsymbol{\psi}^{(k)} \mid u_{i}\right)}{n \rho_{j}^{(k+1)}}
$$

$\mathrm{e}$

$$
\widehat{\Sigma}_{j}^{(k+1)}=\frac{\sum_{i=1}^{n}\left(u_{i}-\boldsymbol{\mu}_{j}^{(k+1)}\right)\left(u_{i}-\boldsymbol{\mu}_{j}^{(k+1)}\right)^{T} \tau_{j}\left(\boldsymbol{\psi}^{(k)} \mid u_{i}\right)}{n \rho_{j}^{(k+1)}},
$$

para $j=1, \ldots, g($ MCLACHLAN; PEEL, 2000).

Os dois passos do algoritmo EM são repetidos de forma alternada até a sua convergência. Utilizam-se critérios de erro na análise da convergência, como os critérios baseados no erro absoluto relativo entre passos sucessivos dos estimadores ou da verossimilhança (ver Seção 2.1.1).

Como comentado na Seção 2.1.2, a convergência do EM é afetada sensivelmente pelo tipo de distribuição e inicialização utilizados. Muitas versões foram propostas para contornar este problema. Um exemplo é o método SEM , cujos conceitos básicos são apresentados na próxima seção.

\subsection{O Algoritmo SEM}

O algoritmo SEM (Stochastic Expectation-Maximization) é uma versão estocástica do algoritmo EM na aplicação a mistura de distribuições (MCLACHLAN; KRISHNAN, 1997). Algumas vantagens do algoritmo SEM são: possui um passo estocástico de simples cômputo, ocasionando baixo custo computacional adicional quando comparado com o EM; evita a convergência do método para o máximo local quando o modelo possui distribuições com solução analítica tratável.

Além disso, com o método SEM, é possível evitar a necessidade de resolver em forma fechada a maximização da esperança condicional $Q\left(\boldsymbol{\theta} ; \boldsymbol{\theta}^{(k)}\right)$ (ver Equação $\left.(2.25)\right)$ (MOSER; ZERUBIA; SERPICO, 2006). Pode-se, então, substituir os estimadores de máxima ve- 
rossimilhança por estimadores de outros métodos de estimação no passo-M do SEM, desde que as propriedades de convergência sejam satisfeitas. Por exemplo, em Moser, Zerubia e Serpico (2006), os estimadores foram definidos com o método MoLC (Method of LogCumulants). Este método utiliza a transformada de Mellin e momentos amostrais para obter os estimadores dos parâmetros de uma distribuição.

Essa flexibilidade no passo-M do algoritmo SEM contribui para a sua aplicação a problemas envolvendo distribuições difíceis de serem tratadas analiticamente, como ocorre com as distribuições $\mathcal{K}$ e $\mathcal{G}^{0}$ polarimétricas. Por isso, nesta tese, o algoritmo SEM em conjunto com os estimadores obtidos pelo método dos momentos foram utilizados para segmentar as imagens polarimétricas multi-look. A próxima seção apresenta a definição do algoritmo SEM .

\subsubsection{Definição}

O algoritmo SEM é uma versão estocástica do EM que restaura as propriedades do conjunto de rótulos não observados $\boldsymbol{r}$. Ele é um caso particular do algoritmo MCEM (Monte Carlo EM) com uma única replicação (MCLACHLAN; KRISHNAN, 1997).

Na Seção 2.2.2, considerou-se $\boldsymbol{u}_{c}=(\boldsymbol{u}, \boldsymbol{r})$ como o conjunto de dados completos contendo a amostra observada $\boldsymbol{u}=\left(u_{1}, \ldots, u_{n}\right)$ e os dados não observados $\boldsymbol{r}=\left(\boldsymbol{r}_{1}, \ldots, \boldsymbol{r}_{n}\right)$. Cada rótulo é um vetor binário $\boldsymbol{r}_{i}=\left(r_{1 i}, \ldots, r_{g i}\right)$ contendo valor $r_{j i}=1$ quando a distribuição $D_{j}$ estiver associada à observação $u_{i}$ (ver Equação (2.15) da página 29).

No algoritmo EM, a informação considerada em cada $r_{j i}$ do vetor de rótulos é a probabilidade a posteriori $\tau_{j i}$ da distribuição $D_{j}$ dada a observação $u_{i}$. O algoritmo SEM acrescenta um passo de amostragem ao EM que mapeia o vetor de probabilidades a posteriori para o vetor binário. Por exemplo, o vetor de probabilidades $\left(\tau_{1 i}, \ldots, \tau_{j i}, \ldots, \tau_{g i}\right)$ é mapeado para o vetor de rótulos $\left(r_{1 i}=0, \ldots, r_{j i}=1, \ldots, r_{g i}=0\right)$ se a distribuição $D_{j}$ for associada a observação $u_{i}$ no passo de amostragem.

Partindo do conjunto paramétrico inicial $\boldsymbol{\psi}^{(0)}$ e considerando $1 \leq i \leq n$ e $1 \leq j \leq g$, com $n$ o tamanho da amostra e $g$ o número de distribuições, o algoritmo SEM consiste de três passos por iteração (CELEUX; DIEBOLT, 1985):

- Passo-E: Calcula as probabilidades a posteriori, dado o conjunto paramétrico atual $\boldsymbol{\psi}^{(k)}$. Para cada observação $u_{i}$, calcular o vetor $\left(\tau_{1 i}^{(k)}, \ldots, \tau_{j i}^{(k)}, \ldots, \tau_{g i}^{(k)}\right)$, no qual a probabilidade $\tau_{j i}^{(k)}=\tau_{j}\left(\boldsymbol{\psi}^{(k)} \mid u_{i}\right)$ está definida na Equação (2.24) (página 31). 
- Passo-S: Amostra, para cada observação $u_{i}$, uma realização $\omega_{i}$ em $\{1, \ldots, g\}$ e associa $r_{j i}=1$ para $j=\omega_{i}$ e $r_{j i}=0$ no caso contrário. O processo de amostragem é realizado com a distribuição multinomial em $g$ categorias com probabilidades $\boldsymbol{\tau}_{i}^{(k)}=\left(\tau_{1 i}^{(k)}, \ldots, \tau_{g i}^{(k)}\right)$, ou seja, $R_{1}, \ldots, R_{n} \sim \mathcal{M}_{g}\left(1, \boldsymbol{\tau}_{i}^{(k)}\right)$, contendo a função de probabilidade definida pela Equação (2.16) (página 29). Como discutido na Seção 2.2.2, os rótulos $\left(\boldsymbol{r}_{1}, \ldots, \boldsymbol{r}_{n}\right)$ são modelados pela distribuição multinomial. Com o mapeamento obtido pelo processo de amostragem, apenas um rótulo $r_{j i}$ é associado a cada $u_{i}$, resultando numa partição em que o conjunto observado $\boldsymbol{u}$ é dividido em $g$ grupos $\left(\Omega_{1}^{(k)}, \ldots, \Omega_{g}^{(k)}\right)$. A partição é definida por $u_{i} \in \Omega_{j}^{(k)} \Leftrightarrow r_{j i}=1$.

- Passo-M: Atualiza os parâmetros da mistura $\boldsymbol{\psi}^{(k+1)}$ baseado no algoritmo EM. Contudo, as probabilidades a posteriori $\tau_{j i}^{(k)}$ são substituídas pelos vetores binários $r_{j i}$, tornando as atualizações dependentes da partição $\left(\Omega_{1}^{(k)}, \ldots, \Omega_{g}^{(k)}\right)$. Considerando a Equação (2.27) (página 31), as proporções são atualizadas por

$$
\widehat{\rho}_{j}^{(k+1)}=\frac{\# \Omega_{j}^{(k)}}{n}, j=1, \ldots, g,
$$

em que $\# \Omega_{j}$ é a cardinalidade do grupo $\Omega_{j}$. No exemplo de mistura de distribuições gaussianas multivariadas, os parâmetros são $\boldsymbol{\theta}_{j}=\left(\boldsymbol{\mu}_{j}, \Sigma_{j}\right)$, sendo $\boldsymbol{\mu}_{j}$ o vetor média e $\Sigma_{j}$ a matriz de covariância da $j$-ésima componente. As atualizações são

$$
\widehat{\boldsymbol{\mu}}_{j}^{(k+1)}=\frac{\sum_{i \in \Omega_{j}^{(k)}} u_{i}}{\# \Omega_{j}^{(k)}}
$$

e

$$
\widehat{\Sigma}_{j}^{(k+1)}=\frac{\sum_{i \in \Omega_{j}^{(k)}}\left(u_{i}-\boldsymbol{\mu}_{j}^{(k+1)}\right)\left(u_{i}-\boldsymbol{\mu}_{j}^{(k+1)}\right)^{T}}{\# \Omega_{j}^{(k)}},
$$

para $j=1, \ldots, g$.

Os passos do algoritmo SEM são repetidos de forma alternada até a sua convergência. Utilizam-se critérios de erro na análise da convergência, como os comentados na Seção 2.1.1.

A próxima seção apresenta a aplicação dos modelos de mistura na segmentação de imagens. 


\subsection{Aplicação à Segmentação de Imagens}

Os modelos de mistura de distribuições são aplicados à segmentação de imagens quando utilizados como técnicas de agrupamento de dados (DUDA; HART; STORK, 2001). Nesse contexto, cada distribuição $D_{j}$ que compõe o modelo de mistura $M(\boldsymbol{\theta}, \boldsymbol{\rho})$ (ver Equação (2.8) da página 26) modela um segmento (grupo $\Omega_{j}$ ) a ser definido na imagem.

Inicialmente, as estimativas dos parâmetros do modelo de mistura são calculadas utilizando o algoritmo EM ou extensões. Este processamento define a distribuição de cada segmento (região) na imagem. Em seguida, os pixels da imagem são associados ao segmento respectivo de acordo com uma regra de decisão estabelecida e as estimativas calculadas no processamento anterior.

Em Delignon, Marzouki e Pieczynski (1997), as técnicas de segmentação de imagens utilizando mistura de distribuições são associadas a duas abordagens: análise pontual (chamada de "cega") e contextual (chamada de "global"). Na segmentação pontual, os pixels são agrupados de forma independente, ou seja, desconsiderando as informações dos pixels vizinhos. Neste caso, o algoritmo EM e extensões são aplicados diretamente. Na segmentação contextual, assume-se uma dependência espacial entre o pixel e seus vizinhos. Há, então, a inclusão do conceito de campos aleatórios Markovianos ao método EM, em que todos os tipos de cliques (combinações possíveis a uma certa vizinhança) são analisados. A segmentação contextual pode resultar num alto custo computacional. Algumas aplicações envolvendo a segmentação contextual de mistura de distribuições estão descritas em Delignon, Marzouki e Pieczynski (1997) e em Alfò, Nieddu e Vicari (2008).

Nesta tese, adotou-se a abordagem "cega". A regra de decisão utilizada foi o critério definido com as probabilidades a posteriori (MCLACHLAN; PEEL, 2000). Considerando que o atributo do $i$-ésimo pixel da imagem é a ocorrência de uma variável aleatória $U_{i}$ seguindo o modelo de mistura $M(\boldsymbol{\theta}, \boldsymbol{\rho})$ composto de $g$ distribuições (segmentos), tem-se que o dado observado (atributo) $u_{i}$ irá pertencer ao segmento $\Omega_{j}$ se

$$
\tau_{j}\left(\boldsymbol{\psi}_{j} \mid u_{i}\right)>\tau_{h}\left(\boldsymbol{\psi}_{h}, \mid u_{i}\right) \rightarrow u_{i} \in \Omega_{j}, j \neq h \text { e } \forall j, h=1, \ldots, g
$$

no qual $\left(\Omega_{1}, \ldots, \Omega_{g}\right)$ indicará a partição obtida e $\tau_{j}\left(\boldsymbol{\psi}_{j} \mid u_{i}\right)$ está definido na Equação $(2.24)$ (página 31). Considerou-se, nesta tese, a indexação dos pixels pelo ordenamento lexicográfico. Os dados da imagem são representados pelo vetor $\left(u_{1}, \ldots, u_{n}\right)$ de tamanho $n=L \cdot C$ ao invés da matriz de dimensão $L \times C$. 
O próximo capítulo apresenta as distribuições utilizadas nas imagens SAR polarimétricas multi-look. 


\section{Capítulo 3}

\section{Modelos para Dados PolSAR multi-look}

Esta tese está focada nos modelos destinados aos dados SAR polarimétricos multi-look com o intuito de aplicá-los à segmentação de imagens. Portanto, este capítulo aprofunda os estudos sobre os modelos utilizados a estes tipos de dados, como apresentados nas próximas seções.

As seções iniciais apresentam alguns conceitos introdutórios sobre as imagens SAR e PolSAR multi-look. O processo de aquisição das imagens SAR e conceitos relacionados são muito extensos, como encontrado em Lee e Pottier (2009), Oliver e Quegan (1998) e Ulably e Elachi (1990). Por isso, a próxima seção apresenta um estudo direcionado sobre esses conceitos introdutórios.

\subsection{Imagens SAR}

As pesquisas utilizando as imagens SAR continuam em crescente avanço no Sensoriamento Remoto (LEE; POTTIER, 2009). Estas imagens são adquiridas por radares que atuam na faixa de micro-ondas e, por causa disso, apresentam informações adicionais às imagens óticas e com propriedades importantes de acordo com as características do sensor e parâmetros do sistema SAR adotados.

Alguns exemplos de dados SAR são: intensidade e amplitude (dados reais) e polarimétricos e interferométricos (dados complexos contendo amplitude e fase) (LEE; POTTIER, 2009; OLIVER; QUEGAN, 1998). 
As vantagens dos sistemas SAR são: independência de luz solar, permitindo a aquisição em qualquer horário do dia por possuir fonte de iluminação própria; independência do clima, permitindo a aquisição das imagens em dias nublados; adição de informação, fornecendo propriedades dielétricas e geométricas das superfícies observadas e confiança no dados por conseguir penetrar mais na superfície quando a faixa do micro-ondas é comparada com as outras faixas do espectro eletromagnético (OLIVER; QUEGAN, 1998; TSO; MATHER, 2001).

A desvantagem das imagens SAR é a presença do ruído multiplicativo speckle. Este ruído é inerente ao processo de aquisição e formação dos dados, fornecendo imagens com aspecto granuloso. Este fato dificulta tanto a interpretação das imagens quanto o seu processamento e análise.

Por causa das dificuldades encontradas na análise de imagens SAR, o avanço das pesquisas envolvendo as propriedades estatísticas da imagem é crescente. Em especial, no caso de imagens polarimétricas multi-look, alguns modelos importantes a estes tipos de dados são discutidos em Jacobo-Berlles (2005), Bombrun e Beaulieu (2008), Freitas, Frery e Correia (2005), Lee, Grunes e Kwok (1994) e Lee et al. (1994b).

As próximas seções fornecem alguns conceitos introdutórios.

\subsubsection{Sistema SAR e Propriedades}

Nos sistemas de radar (Radio Detection And Ranging) de visada lateral, o processo de aquisição da imagem começa com a emissão de radiação eletromagnética polarizada em direção à superfície terrestre. A radiação é emitida na faixa de micro-ondas pela antena do radar. Quando a radiação incide sobre a superfície sofre um processo de espalhamento, resultando numa emissão da radiação em todas as direções. Uma porção dessa energia espalhada é captada pela antena do radar, chamada de energia retroespalhada. Esse é o princípio básico de funcionamento de um radar que pode ser transportado por uma aeronave (radares aerotransportados) ou por um satélite (OLIVER; QUEGAN, 1998; TSO; MATHER, 2001).

Os radares de visada lateral são: radar de abertura real (SLAR - Side Looking Airbone Radar, RAR - Real Aperture Radar) e radar de abertura sintética (SAR - Synthetic Aperture Radar). Um fator que diferencia esses dois tipos de radares é o tamanho da antena que influencia na abertura de irradiação da antena do radar e, por conseguinte, a resolução espacial da imagem. Nos radares SLAR-RAR, a abertura de irradiação é limitada pelo 
tamanho real da antena do radar. Estes radares não suportam uma antena muito grande por inviabilidade de transporte.

Os radares SAR contornam essa limitação utilizando um sistema que forma uma antena virtual. No deslocamento da plataforma, a antena é simulada com a aquisição dos sinais retroespalhados de uma cena analisada durante um intervalo de tempo. Os sinais de retorno são obtidos utilizando mecanismos de gravação e processamento de dados (RENNÓ, 1995; TSO; MATHER, 2001).

No processo de aquisição das imagens SAR, o sinal retroespalhado (dados de retorno) é influenciado por alguns parâmetros do sistema de radar, como o comprimento de onda e o coeficiente de retroespalhamento (TSO; MATHER, 2001). As faixas de comprimento de onda definem as bandas do radar e influenciam na profundidade de penetração do alvo analisado. Por exemplo, a banda $L$ com comprimento de onda de $25 \mathrm{~cm}$ apresenta maior profundidade de penetração do alvo analisado que a banda $C$ com comprimento de onda de $5 \mathrm{~cm}$ (OLIVER; QUEGAN, 1998).

O coeficiente de retroespalhamento é influenciado pela rugosidade superficial do alvo e por suas condições dielétricas. Estas propriedades incidem na intensidade do sinal de retorno. Outro fator importante é o ângulo de incidência do feixe. Quanto maior for o ângulo de incidência, menor o pulso de retorno (RENNÓ, 1995).

A rugosidade superficial é uma propriedade importante que influencia a parcela refletida pelo sinal (TSO; MATHER, 2001). Esta propriedade depende da variação superficial do conjunto de objetos iluminados e está relacionada com a reflexão da radiação eletromagnética pela superfície. Quanto maior a rugosidade mais difusa é a radiação espalhada, ou seja, não existe uma direção preferencial para os feixes refletidos e a energia é espalhada em todas as direções, chamada de energia difusa.

Em superfícies lisas, consideradas regiões mais homogêneas, toda a radiação incidente é refletida com ângulo igual ao ângulo de incidência, porém em direção contrária. Portanto, não há retorno do pulso a ser emitido para o radar. Este fenômeno é chamado de reflexão especular. Quando a reflexão especular ocorre, o sinal é totalmente perdido. Este fato gera um retroespalhamento fraco e resulta num coeficiente de retroespalhamento com valor baixo. Em geral nas imagens SAR, as tonalidades escuras estão associadas a regiões mais homogêneas, como o rio.

Em áreas mais heterogêneas, como as áreas urbanas que são consideradas extremamente heterogêneas, ocorre o fenômeno de reflexão múltipla: a energia incidente é refletida por diferentes superfícies, por causa da rugosidade, retornando pulso ao radar. Este fenômeno gera um retroespalhamento forte e ocasiona manchas claras na imagem.

Outro aspecto a ser considerado na aquisição das imagens é a polarização. A polari- 
zação descreve a orientação do campo elétrico de uma onda eletromagnética (ULABLY; ELACHI, 1990). Para as imagens de intensidade e amplitude, os sistemas de radar utilizam polarizações lineares paralelas, $h h$ e $v v$, ou cruzadas, $h v$ e $v h$. As polarizações da onda transmitida e recebida estão indicadas pela primeira e segunda letra de cada componente, respectivamente. Por exemplo, a componente $h v$ indica a transmissão de uma onda polarizada na horizontal e o recebimento da onda polarizada na vertical pelo radar (RENNÓ, 1995; TSO; MATHER, 2001).

Com os radares polarimétricos é possível definir os retornos individuais em cada polarização da onda transmitida e recebida, formando uma matriz de espalhamento complexa dos alvos (scattering matrix complex) e definida por

$$
\boldsymbol{S}=\left(\begin{array}{cc}
S_{h h} & S_{h v} \\
S_{v h} & S_{v v}
\end{array}\right)
$$

na qual os retornos $S_{h h}$ e $S_{v v}$ são chamados de co-polarizados, enquanto os retornos $S_{h v}$ e $S_{v h}$ são chamados de componentes de polarização cruzadas (JACOBO-BERLLES, 2005). Segundo Rennó (1995), o grau de penetração da onda num alvo é influenciado pelo tipo de polarização utilizada pelo sistema.

No caso de radares mono-estáticos, ou mono-polarizados, utiliza-se a mesma antena para emitir e receber o sinal, resultando na igualdade entre as polarizações cruzadas, ou seja, $S_{h v}=S_{v h}($ ULABLY; ELACHI, 1990).

Além da matriz espalhamento $\boldsymbol{S}$, existem outras formas de se representar os dados polarimétricos, algumas delas são: matriz de Mueller e Mueller modificada, matriz de Stokes, matriz de covariância e matriz de coerência (LEE; POTTIER, 2009; TOUZI et al., 2004; ULABLY; ELACHI, 1990). Nesta tese, utiliza-se a matriz de covariância na representação de imagens polarimétricas multi-look.

\subsubsection{Ruído Speckle}

As imagens SAR são corrompidas por um ruído dependente do sinal chamado speckle. Nos sistemas SAR, os dados das imagens são obtidos com a soma coerente (vetorial) do sinal retornado por cada um dos elementos difusores que compõem uma célula de resolução da cena analisada. Como os elementos difusores estão distribuídos de forma aleatória, a soma vetorial dos sinais de retorno sofre interferências tanto construtivas quanto destrutivas, resultando em imagens com aspecto granuloso. Esta característica nas imagens SAR 
dificulta a sua interpretação e processamento (RENNÓ, 1995).

Há duas abordagens para atenuar do ruído speckle: o processamento multi-look e a filtragem. O processamento multi-look resulta na média dos dados obtidos em cada look (visada). A abertura sintética da antena é dividida em setores adjacentes (looks) que fornecem dados da mesma cena analisada. Este processamento reduz o efeito do speckle e, também, diminui a resolução espacial na direção azimute ${ }^{1}$. Por exemplo, uma imagem SAR de 4-looks possui o ruído reduzido quando comparada com uma imagem de 1-look para a mesma cena, mas a resolução azimutal na imagem de 4-looks também é inferior à imagem de 1-look (RENNÓ, 1995; TSO; MATHER, 2001). O processamento multi-look é discutido na seção 3.3 .

Segundo Lee e Pottier (2009), os filtros aplicados a imagens PolSAR devem preservar as propriedades polarimétricas e, também, devem ser adaptativos para preservar as características de dispersão. Em Lee e Pottier (2009) há uma revisão sobre o assunto, abordando alguns filtros como os propostos em Vasile et al. (2006) e em Lee et al. (2006). Uma contribuição recente é o filtro sigma melhorado proposto em Lee et al. (2009).

\subsection{Imagens PolSAR}

Nas imagens SAR polarimétricas single-look, cada coordenada do pixel é formada por uma matriz de espalhamento complexa $\boldsymbol{S}$ da Equação (3.1), cujos dados são obtidos pela soma dos retornos individuais em cada polarização $(h h, h v, v h, v v)$. Nos radares monopolarizados, as polarizações cruzadas são iguais $\left(S_{h v}=S_{v h}\right)$, ou seja, os dados de cada pixel da imagem polarimétrica single-look são definidos por

$$
\boldsymbol{S}=\left[S_{h h}, S_{h v}, S_{v v}\right]^{t}
$$

em que,

$$
S_{p q}=\left|S_{p q}\right| e^{i \phi_{p q}}=\sum_{k=1}^{N}\left|S_{p q}^{k}\right| e^{i \phi_{p q}^{k}}
$$

e $N$ é o número de dispersores de uma célula de resolução (cada um possuindo uma amplitude $\left|S_{p q}^{k}\right|$ e uma fase $\phi_{p q}^{k}$ ), $p q=\{h h, h v, v v\}$ são as polarizações e $i=\sqrt{-1}$ (FREITAS; FRERY; CORREIA, 2005; OLIVER; QUEGAN, 1998).

Nos sistemas SAR polarimétricos multi-look, o processamento dos radares consiste em,

\footnotetext{
${ }^{1} \mathrm{~A}$ direção azimute é a direção de deslocamento da plataforma que transporta o radar, ou seja, corresponde a direção de voo.
} 
primeiro, dividir a abertura sintética da antena em alguns setores (looks), segundo, processá-los de forma independente e, por último, somar todas as informações obtidas no processamento. Este procedimento reduz a presença do ruído speckle na imagem, mas, também, diminui da resolução azimutal (RENNÓ, 1995).

As imagens PolSAR multi-look também podem ser obtidas com as imagens polarimétricas single-look (FREITAS; FRERY; CORREIA, 2005). Neste processamento, utiliza-se a vizinhança do pixel na imagem (considerados independentes) para formar a matriz de covariância complexa $\eta$-look, definida por

$$
\boldsymbol{Z}=\frac{1}{\eta} \sum_{k=1}^{\eta} \boldsymbol{S}(k) \boldsymbol{S}^{* t}(k)=\left(\begin{array}{ccc}
Z_{h h} & Z_{h h h v} & Z_{h h v v} \\
Z_{h h h v}^{*} & Z_{h v} & Z_{h v v v} \\
Z_{h h v v}^{*} & Z_{h v v v}^{*} & Z_{v v}
\end{array}\right)
$$

na qual, * é o conjugado e $t$ é a transposta. A matriz $Z$ é hermitiana e sua diagonal principal contém as intensidades. Cada coordenada da imagem polarimétrica $\eta$-look possui uma matriz $\boldsymbol{Z}$, ou seja, é um dado multivariado composto de nove informações.

As próximas seções apresentam alguns modelos para dados PolSAR multi-look.

\subsection{Modelo Multiplicativo}

O modelo multiplicativo é muito utilizado na obtenção de distribuições que ajustam os dados de imagens SAR (OLIVER; QUEGAN, 1998). Ele se baseia numa interpretação física para modelar os dados: a imagem é corrompida pelo ruído speckle, sendo este um ruído dependente do sinal por causa da radiação coerente incidente sobre a superfície observada. Como o speckle é um ruído multiplicativo, não é adequado utilizar a distribuição gaussiana na descrição dos dados de imagens PolSAR multi-look (FRERY et al., 1997).

De acordo com o modelo multiplicativo, cada dado da imagem SAR é a ocorrência de uma variável aleatória $Z$ que é definida pelo produto de duas variáveis aleatórias independentes $(Z=X Y): X$ modelando o retroespalhamento (backscatter) e $Y$ modelando o ruído speckle. A variável aleatória $X$ é real positiva e a variável aleatória $Y$ pode ser complexa ou real positiva, adotando tanto a forma matricial quanto escalar. Por exemplo, nas imagens de amplitude ou intensidade, a variável aleatória $Y$ é real positiva e escalar. As distribuições atribuídas as variáveis aleatórias $X$ e $Y$ variam de acordo com o tipo de imagem SAR e o grau de homogeneidade das regiões (FREITAS; FRERY; CORREIA, 2005). 
No caso de imagens SAR polarimétricas multi-look, o retorno de cada dado da imagem é definido por:

$$
\boldsymbol{Z}=X \boldsymbol{Y}
$$

em que a variável aleatória $\boldsymbol{Y}$ modela os dados do speckle que é a matriz de covariância complexa $\eta$-look da Equação (3.4), enquanto que a variável aleatória $X$ que modela o retroespalhamento é real positiva com $\mathbb{E}(X)=1$.

Os modelos do speckle, do retroespalhamento e do retorno das imagens PolSAR multilook estão discutidos nas próximas seções. Os modelos definidos ao retroespalhamento variam de acordo com as características da imagem: homogêneas, heterogêneas ou extremamente heterogêneas.

\subsection{Modelo para o Ruído Speckle}

Nas imagens polarimétricas multi-look e assumindo o modelo multiplicativo, os dados do ruído speckle é uma matriz de covariância complexa $\eta$-look de tamanho $m \times m$, sendo $m$ é o número de polarizações (ver o exemplo da Equação (3.4) com $m=3$ polarizações).

Os dados do ruído speckle são ocorrências de variáveis aleatórias $\boldsymbol{Y}$ que seguem a distribuição Wishart Complexa Centrada (LEE; GRUNES; KWOK, 1994; LEE et al., 1994a). Denotando $\boldsymbol{Y} \sim W(C, \eta)$, o conjunto paramétrico da distribuição Wishart é composto por matriz hermitiana $(C)$ e pelo número de looks $(\eta)$ que indica os graus de liberdade da distribuição.

A função densidade de probabilidade da distribuição Wishart é definida por

$$
f_{\boldsymbol{Y}}(\boldsymbol{y})=\frac{\eta^{\eta m}|\boldsymbol{y}|^{\eta-m}}{h(\eta, m)|C|^{\eta}} \exp \left(-\eta \operatorname{Tr}\left(C^{-1} \boldsymbol{y}\right)\right), \quad \eta \geq m
$$

na qual Tr e $|\cdot|$ denotam o traço e o determinante da matriz, respectivamente, e $\mathbb{E}(\boldsymbol{Y})=C$. A função escala $h(\eta, m)$ é definida por

$$
h(\eta, m)=\pi^{\frac{m(m-1)}{2}} \Gamma(\eta) \cdots \Gamma(\eta-m+1)
$$

com $\Gamma($ ) denotando a função Gama (FREITAS; FRERY; CORREIA, 2005). 


\subsection{Modelos para a Retroespalhamento}

Quando o modelo multiplicativo é aplicado a imagens polarimétricas multi-look, as informações do retroespalhamento (backscatter) são ocorrências de uma variável aleatória $X$ que controla as flutuações do valor médio (definido pela matriz de covariância $C$ ). Esta característica é influenciada pela variabilidade do alvo analisado, na qual regiões mais homogêneas possuem poucas flutuações em função do valor médio, enquanto alvos mais heterogêneos apresentam flutuações variadas. Como consequência, há uma variedade de distribuições que modelam o retroespalhamento de acordo com as características do alvo analisado, resultando em diferentes modelos de retorno.

Jakeman e Pusey (1976) utilizaram a distribuição Gama (denotada por $\Gamma(\alpha, \alpha)$ ) para modelar o retroespalhamento de imagens SAR univariadas, como as imagens de amplitude e intensidade, contendo regiões heterogêneas como vegetações. Em Lee et al. (1994b), esta distribuição do retroespalhamento foi aplicada a imagens polarimétricas multi-look.

Frery et al. (1997) utilizaram a distribuição Recíproca da Gama $\left(\Gamma^{-1}(\alpha,(-\alpha-1))\right)$ para modelar o retroespalhamento de imagens SAR univariadas contendo regiões mais heterogêneas como as áreas urbanas, chamadas de extremantente heterogêneas. Em Correia (1998), esta distribuição do retroespalhamento foi aplicada a imagens polarimétricas multi-look.

Muller et al. (2000) utilizaram a distribuição Gaussiana inversa com média unitária (denotada por $\operatorname{GI}(\omega, 1)$ ) para modelar o retroespalhamento de imagens SAR univariadas contendo regiões extremamente heterogêneas. Em Jacobo-Berlles (2005), esta distribuição do retroespalhamento foi aplicada a imagens polarimétricas multi-look.

Em Frery et al. (1997), a distribuição Gaussiana inversa generalizada (denotada por $\left.\mathrm{N}^{-1}(\alpha, \omega)\right)$ também foi proposta aos dados do retoespalhamento de imagens SAR univariadas. Esta distribuição é um caso geral dos modelos discutidos anteriormente. Em Freitas, Frery e Correia (2005), esta distribuição do retroespalhamento foi aplicada a imagens polarimétricas multi-look.

O problema de se trabalhar com a distribuição Gaussiana inversa generalizada resulta no fato dela ser muito difícil de ser manipulada (FREITAS; FRERY; CORREIA, 2005). Trabalham-se, então, com os casos particulares desta distribuição, que são: a distribuição Gama, a distribuição Recíproca da Gama e a distribuição Gaussiana inversa com média unitária. Uma forma simplificada de mostrar a relação entre a Gaussiana inversa genera- 
lizada e estes três casos particulares é:

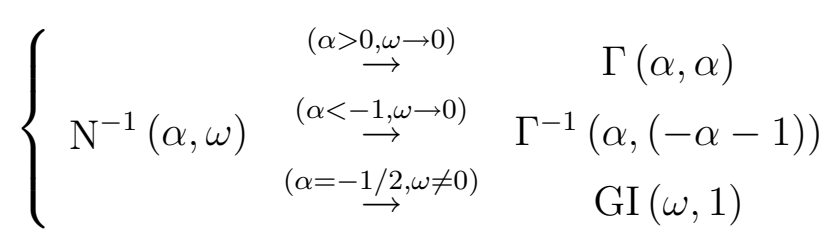

Em Tison et al. (2004), a distribuição Fisher foi proposta para ajustar os dados do retroespalhamento de imagens SAR univariadas contendo regiões extremamente heterogêneas. Em Bombrun e Beaulieu (2008), esta distribuição do retroespalhamento foi aplicada a imagens PolSAR multi-look.

Algumas distribuições do retroespalhamento são discutidas nas próximas seções.

\subsubsection{Distribuição Gama: $\Gamma(\alpha, \alpha)$}

Assumindo o modelo multiplicativo, as informações do retroespalhamento são ocorrências da variável aleatória $X$ que segue a distribuição Gama com média unitária (FREITAS; FRERY; CORREIA, 2005). Neste caso, $\mathrm{X} \sim \Gamma(\alpha, \alpha)$ e sua função densidade de probabilidade é definida por

$$
f_{X}(x)=\frac{\alpha^{\alpha} x^{\alpha-1}}{\Gamma(\alpha)} \exp (-\alpha x), \quad \alpha, x>0
$$

na qual, $\mathbb{E}(X)=\alpha / \alpha=1$ (DEGROOT; SCHERVISH, 2002).

\subsubsection{Distribuição Recíproca da Gama: $\Gamma^{-1}(\alpha,(-\alpha-1))$}

Assumindo o modelo multiplicativo, as informações do retroespalhamento são ocorrências da variável aleatória $X$ seguindo a distribuição recíproca da Gama com média unitária (FREITAS; FRERY; CORREIA, 2005). Neste caso, $\mathrm{X} \sim \Gamma^{-1}(\alpha,(-\alpha-1))$ e sua função densidade de probabilidade é definida por

$$
f_{X}(x)=\frac{x^{\alpha-1}}{(-\alpha-1)^{\alpha} \Gamma(-\alpha)} \exp \left(\frac{\alpha+1}{x}\right), \quad-\alpha, x>0
$$

na qual $\mathbb{E}(X)=(-\alpha-1) /(-\alpha-1)=1$. 


\subsection{Modelo para o Retorno}

Como discutido nas seções anteriores, algumas distribuições foram propostas a imagens PolSAR multi-look com base no modelo multiplicativo. Em cada pixel da imagem, a matriz de covariância complexa $\eta$-look (com variável aleatória $\boldsymbol{Z}=X \boldsymbol{Y}$ ) é definida com a distribuição que modela do ruído speckle $(\boldsymbol{Y})$ e com a distribuição que modela o retroespalhamento $(X)$. A distribuição Wishart modela o speckle (ver Seção 3.4) enquanto que há uma variedade de distribuições que modelam o retroespalhamento (ver Seção 3.5). As distribuições do retroespalhamento diferenciam de acordo com o grau de homogeneidade do alvo a que foram propostas, ou seja, se foram aplicadas a alvos mais homogêneos, heterogêneos ou extremamente heterogêneos. Esta variedade de distribuições do retroespalhamento fornece diferentes distribuições de retorno. Os textos de Correia (1998), Freitas, Frery e Correia (2005) e Jacobo-Berlles (2005) fornecem revisões bibliográficas de algumas distribuições que modelam dados PolSAR.

A clássica distribuição Wishart é a mais aplicada ao processamento e análise de imagens PolSAR multi-look (LEE; GRUNES; KWOK, 1994; LEE et al., 1994a; LEE; POTTIER, 2009). A distribuição do retorno, denotada por $\boldsymbol{Z} \sim W(C, \eta)$, é definida pela distribuição do speckle, pois a distribuição do retroespalhamento é uma função constante $X=1$.

Jakeman e Pusey (1976) utilizaram a distribuição Gama para modelar o retroespalhamento $(X \sim \Gamma(\alpha, \alpha))$ com o intuito de derivar a distribuição $\mathcal{K}$ para o retorno de alvos heterogêneos. Em Lee et al. (1994b), este modelo foi estendido a imagens polarimétricas multi-look, no qual $\boldsymbol{Z} \sim \mathcal{K}_{p}(\alpha, C, \eta)$.

Frery et al. (1997) propuseram uma distribuição mais geral ao retorno, chamada de $\mathcal{G}$, para imagens complexas univariadas e de amplitude e intensidade multi-look (denotadas por $\mathcal{G}_{A}$ e $\mathcal{G}_{I}$ ). No caso, a variável aleatória do retroespalhamento segue uma distribuição Gaussiana inversa generalizada, denotada por $X \sim \mathrm{N}^{-1}(\alpha, \omega)$. O intuito foi fornecer um modelo ajustável tanto para regiões homogêneas, quanto heterogêneas e extremanente heretorêgeneas, como as áreas urbanas. O problema é que esta distribuição é difícil de ser tratada analiticamente como, por exemplo, na estimação dos parâmetros por máxima verossimilhança.

Para contornar esta situação, Frery et al. (1997) também propuseram um caso particular à distribuição $\mathcal{G}$, chamada de $\mathcal{G}^{0}$. No caso, as ocorrências do retroespalhamento contêm variáveis aleatórias seguindo a distribuição recíproca da Gama, $X \sim \Gamma^{-1}(\alpha,(-\alpha-1))$. O artigo também mostra a relação entre a distribuição $\mathcal{G}$ e os seus casos particulares: distribuição $\mathcal{G}^{0}, \mathcal{K}$ e Gaussiana ou Gama ou Raiz da Gama (no ajuste de regiões ho- 
mogêneas para dados complexos univariados, de intensidade ou amplitude, respectivamente). Em Correia (1998), a distribuição $\mathcal{G}^{0}$ é estendida para as imagens polarimétricas multi-look, denotada por $\mathcal{G}_{P}^{0}(\alpha, C, \eta)$.

Em Freitas, Frery e Correia (2005), a distribuição $\mathcal{G}$ é estendida para as imagens polarimétricas multi-look, denotada por $\mathcal{G}_{P}(\alpha, \omega, C, \eta)$. Além disso, o artigo comenta a relação entre a $\mathcal{G}_{P}$ e as distribuições $\mathcal{G}_{P}^{0}, \mathcal{K}_{P}$ e Wishart.

Outro caso particular da distribuição $\mathcal{G}$ é a distribuição $\mathcal{G}^{\text {Harmonica }}$, chamada de $\mathcal{G}^{H}$ (MULLER et al., 2000). Em Jacobo-Berlles (2005), é proposta a extensão da distribuição $\mathcal{G}^{H}$ para imagens polarimétricas multi-look, chamada de $\mathcal{G}_{P}^{H}$.

Segundo Frery, Correia e Freitas (2007) e Jacobo-Berlles (2005), uma forma simplificada de mostrar a relação entre a distribuição $\mathcal{G}$ e estes quatro casos particulares é:

$$
\left\{\begin{array}{lllll} 
& \stackrel{(\alpha>0, \omega \rightarrow 0)}{\longrightarrow} & \mathcal{K}_{P}(\alpha, C, \eta) & \stackrel{(\alpha \rightarrow \infty)}{\longrightarrow} & W(C, \eta) \\
\mathcal{G}(\alpha, \omega, C, \eta) & \stackrel{(\alpha<-1, \omega \rightarrow 0)}{\longrightarrow} & \mathcal{G}_{P}^{0}(\alpha, C, \eta) & \stackrel{(\alpha \rightarrow-\infty)}{\longrightarrow} & W(C, \eta) \\
& \stackrel{(\alpha=-1 / 2, \omega \neq 0)}{\longrightarrow} & \mathcal{G}_{P}^{H}(\omega, C, \eta) & &
\end{array}\right.
$$

Em Frery, Correia e Freitas (2007), analisou-se a classificação de imagens polarimétricas multi-look completas usando os modelos apresentados em Freitas, Frery e Correia (2005) para diferentes frequências (bandas da imagem). Além disso, as classificações foram realizadas de forma pontual e com uma análise contextual para classificadores de máxima verossimilhança.

Bombrun e Beaulieu (2008) propuseram a distribuição KummerU para modelar regiões mais heterogêneas, como florestas e áreas urbanas. Assumindo o modelo multiplicativo, a distribuição KummerU foi derivada utilizando a distribuição Wishart para modelar o speckle e a distribuição Fisher para modelar o retroespalhamento (TISON et al., 2004).

As próximas seções apresentam algumas distribuições do retorno.

\subsubsection{Distribuição Wishart Complexa: $Z \sim W(C, \eta)$}

A distribuição Wishart é um modelo clássico e muito utilizado no processamento e análise de imagens polarimétricas multi-look (LEE; GRUNES; KWOK, 1994; LEE et al., 1994a; LEE; POTTIER, 2009). Este é o modelo mais simples dentre as distribuições baseadas no modelo multiplicativo a dados PolSAR multi-look. 
Neste modelo, como a variável aleatória do retroespalhamento $X$ é constante e positiva com $\mathbb{E}(X)=1$, a variável aleatória do retorno é definida por $\boldsymbol{Z}=1 \boldsymbol{Y}$ e segue a mesma distribuição que modela o ruído speckle: $\boldsymbol{Z} \sim W(C, \eta)$. A função densidade de probabilidade da distribuição Wishart está definida na Equação 3.6 (página 43). O valor esperado da variável aleatória do retorno é o mesmo da variável aleatória do speckle: $\mathbb{E}(\boldsymbol{Z})=\mathbb{E}(\boldsymbol{Y})=C($ FREITAS; FRERY; CORREIA, 2005).

O modelo Wishart Complexo é característico de regiões mais homogêneas, como as áreas de cultivo.

\subsubsection{Distribuição $\mathcal{K}$ Polarimétrica: $Z \sim \mathcal{K}_{p}(\alpha, C, \eta)$}

A distribuição $\mathcal{K}$ polarimétrica é um modelo clássico que foi proposto para modelar regiões mais heterogêneas em imagens PolSAR multi-look (FRERY; CORREIA; FREITAS, 2007; LEE et al., 1994b).

Considerando o modelo multiplicativo, a variável aleatória do retroespalhamento segue distribuição Gama com média unitária, denotada por $X \sim \Gamma(\alpha, \alpha) \operatorname{com} \alpha>0$. A função densidade de probabilidade da distribuição Gama está definida na Equação 3.9 (página 45). A variável aleatória do ruído speckle segue a distribuição Wishart, denotada por $\boldsymbol{Y} \sim W(C, \eta)$. A função densidade de probabilidade da distribuição Wishart está definida na Equação 3.6 (página 43).

Sejam as distribuições $X \sim \Gamma(\alpha, \alpha)$ e $\boldsymbol{Y} \sim W(C, \eta)$, a variável aleatória do retorno $\boldsymbol{Z} \sim \mathcal{K}_{p}(\alpha, C, \eta)$ possui distribuição com função densidade de probabilidade definida por

$$
f_{\boldsymbol{Z}}(\boldsymbol{z})=\frac{2|\boldsymbol{z}|^{\eta-m}(\eta \alpha) \frac{\alpha+m \eta}{2}}{h(\eta, m)|C|^{\eta} \Gamma(\alpha)}\left(\operatorname{Tr}\left(C^{-1} \boldsymbol{z}\right)\right)^{\frac{\alpha-m \eta}{2}} \mathrm{~K}_{\alpha-m \eta}\left(2 \sqrt{\eta \alpha \operatorname{Tr}\left(C^{-1} \boldsymbol{z}\right)}\right)
$$

com $\alpha>0$ e $\mathrm{K}_{a}(b)$ é a função modificada de Bessel do segundo tipo e ordem $a$. O valor esperado da variável aleatória do retorno é o mesmo da variável aleatória do speckle: $\mathbb{E}(\boldsymbol{Z})=\mathbb{E}(\boldsymbol{Y})=C$.

Segundo Freitas, Frery e Correia (2005), o parâmetro de rugosidade $\alpha$ especifica o grau de homogeneidade do alvo. Sob certas condições, à medida que o parâmetro $\alpha$ aumenta $(\alpha \rightarrow \infty)$, a variável aleatória do retorno segue a distribuição Wishart.

$\mathrm{O}$ modelo $\mathcal{K}_{p}$ descreve regiões heterogêneas, como florestas e vegetação, e, também, regiões homogêneas. 


\subsubsection{Distribuição $\mathcal{G}_{P}^{0}$ Polarimétrica: $Z \sim \mathcal{G}_{P}^{0}(\alpha, C, \eta)$}

A distribuição $\mathcal{G}^{0}$ polarimétrica foi proposta para modelar regiões extremamente heterogêneas em imagens PolSAR multi-look (CORREIA, 1998; FRERY; CORREIA; FREITAS, 2007).

Considerando o modelo multiplicativo, a variável aleatória do retroespalhamento segue distribuição recíproca da Gama com média unitária, denotada por $X \sim \Gamma^{-1}(\alpha,(-\alpha-1))$ com $\alpha<-1$. A função densidade de probabilidade da distribuição recíproca da Gama está definida na Equação 3.10 (página 45). A variável aleatória do ruído speckle segue distribuição Wishart, denotada por $\boldsymbol{Y} \sim W(C, \eta)$. A função densidade de probabilidade da distribuição Wishart está definida na Equação 3.6 (página 43).

Sejam as distribuições $X \sim \Gamma^{-1}(\alpha,(-\alpha-1))$ e $\boldsymbol{Y} \sim W(C, \eta)$, a variável aleatória do retorno $\boldsymbol{Z} \sim \mathcal{G}_{p}^{0}(\alpha, C, \eta)$ possui distribuição com função densidade de probabilidade definida por

$$
f_{\boldsymbol{Z}}(\boldsymbol{z})=\frac{\eta^{m \eta}|\boldsymbol{z}|^{\eta-m} \Gamma(m \eta-\alpha)}{h(\eta, m)|C|^{\eta} \Gamma(-\alpha)(-\alpha-1)^{\alpha}}\left(\eta \operatorname{Tr}\left(C^{-1} \boldsymbol{z}\right)+(-\alpha-1)\right)^{\alpha-m \eta}
$$

com $\alpha<-1$. O valor esperado da variável aleatória do retorno é o mesmo da variável aleatória do speckle: $\mathbb{E}(\boldsymbol{Z})=\mathbb{E}(\boldsymbol{Y})=C$.

Segundo Freitas, Frery e Correia (2005), o parâmetro de rugosidade $\alpha$ especifica o grau de homogeneidade do alvo. Sob certas condições, à medida que o parâmetro $\alpha$ diminui $(\alpha \rightarrow-\infty)$, a variável aleatória do retorno segue a distribuição Wishart.

O modelo $\mathcal{G}_{P}^{0}$ descreve áreas extremamente heterogêneas, como as áreas urbanas, e, também, regiões homogêneas e heterogêneas (FREITAS; FRERY; CORREIA, 2005).

\subsubsection{Interpretação dos Parâmetros}

O conjunto paramétrico $(\alpha, C, \eta)$ das distribuições: $W(C, \eta), \mathcal{K}_{p}(\alpha, C, \eta)$ e $\mathcal{G}_{P}^{0}(\alpha, C, \eta)$ possui um papel importante no ajuste dos dados dos alvos analisados.

O parâmetro $\eta$ corresponde à quantidade de looks em que a imagem foi processada. Considerou-se o mesmo número de looks para todos os pixels da imagem.

A matriz de covariância $C$ define o brilho de cada uma das regiões presentes na 
imagem. Este parâmetro está associado à distribuição do speckle, $\boldsymbol{Y} \sim W(C, \eta)$.

A adaptação do modelo multiplicativo aos dados das imagens polarimétricas multi-look não é imediata. Para preservar as propriedades do modelo multiplicativo, a distribuição do speckle modela informações do retorno que definem o brilho da imagem, enquanto que a distribuição do retroespalhamento modela a variabilidade do alvo analisado.

O parâmetro $\alpha$ está associado à distribuição do retroespalhamento no modelo $\mathcal{K}_{P}$ com $\alpha>0$ e no modelo $\mathcal{G}_{P}^{0}$ com $\alpha<-1$. Ele é uma medida de rugosidade que controla a variabilidade do alvo. No caso, quanto maior for o valor de $|\alpha|$, mais homogênea é a região da imagem (FRERY; CORREIA; FREITAS, 2007).

Para o modelo $\mathcal{K}_{P}$, valores de rugosidade $\alpha$ próximos de zero ajustam dados de regiões heterogêneas, como as florestas. À medida que o valor de $\alpha$ aumenta, mais homogêneas são as regiões, como as regiões de pasto.

No modelo $\mathcal{G}_{P}^{0}$, valores de rugosidade $\alpha$ próximos de -1 ajustam dados de regiões muito heterogêneas, como as áreas urbanas. À medida que o valor de $\alpha$ diminui, o modelo descreve as regiões heterogêneas até chegar ao ajuste das regiões homogêneas.

\subsection{Estimadores para Modelos Polarimétricos}

Na inferência estatística de dados SAR polarimétricos multi-look, a estimação por máxima verossimilhança é difícil de ser tratada analiticamente. Então, utilizam-se os estimadores $(\widehat{\alpha}, \widehat{C}, \widehat{\eta})$ que foram obtidos pelo método dos momentos (FRERY; CORREIA; FREITAS, 2007).

Seja o $r$-ésimo termo do momento amostral de $N$ amostras univariadas de uma área da imagem $\widetilde{\boldsymbol{Z}}=\left(\boldsymbol{Z}_{1}, \ldots, \boldsymbol{Z}_{N}\right)$ definido por

$$
\widehat{m_{r}}(\widetilde{\boldsymbol{Z}})=\frac{1}{N} \sum_{i=1}^{N} \boldsymbol{Z}_{i}^{r}
$$

e $s(\widetilde{\boldsymbol{Z}})$ o desvio padrão amostral, os estimadores pelo método dos momentos estão descritos a seguir.

O número equivalentes de looks amostral $\widehat{\eta}$ é calculado pela média dos canais de 
intensidade de áreas homogêneas

$$
\widehat{\eta}=\frac{\widehat{\eta}_{h h}+\widehat{\eta}_{h v}+\widehat{\eta}_{v v}}{3} \operatorname{com} \widehat{\eta}_{p}=\left(\frac{\widehat{m}_{1}\left(\widetilde{\boldsymbol{Z}}_{p}\right)}{s\left(\widetilde{\boldsymbol{Z}}_{p}\right)}\right)^{2} \text { e } p=\{h h, h v, v v\} \text {, }
$$

assumindo que as amostras seguem distribuição Gama (OLIVER; QUEGAN, 1998). Devese realizar um teste de aderência nas amostras homogêneas com a distribuição Gama, como os testes $\chi^{2}$ ou Kolmogorov-Smirnov (FRERY; CORREIA; FREITAS, 2007).

O valor esperado das distribuições $W(C, \eta), \mathcal{K}_{P}(\alpha, C, \eta)$ e $\mathcal{G}_{P}^{0}(\alpha, C, \eta)$ é o parâmetro matriz de covariância $(C)$ (FRERY; CORREIA; FREITAS, 2007). Então, o seu estimador é

$$
\hat{\boldsymbol{C}}=\widehat{m_{1}}(\widetilde{\boldsymbol{Z}})=\left(\begin{array}{ccc}
\widehat{m}_{1}\left(\widetilde{\boldsymbol{Z}}_{h h}\right) & \widehat{m_{1}}\left(\widetilde{\boldsymbol{Z}}_{h h h v}\right) & \widehat{m_{1}}\left(\widetilde{\boldsymbol{Z}}_{h h v v}\right) \\
\widehat{m_{1}}\left(\widetilde{\boldsymbol{Z}}_{h h h v}^{*}\right) & \widehat{m_{1}}\left(\widetilde{\boldsymbol{Z}}_{h v}\right) & \widehat{m_{1}}\left(\widetilde{\boldsymbol{Z}}_{h v v v}\right) \\
\widehat{m}_{1}\left(\widetilde{\boldsymbol{Z}}_{h h v v}^{*}\right) & \widehat{m}_{1}\left(\widetilde{\boldsymbol{Z}}_{h v v v}^{*}\right) & \widehat{m}_{1}\left(\widetilde{\boldsymbol{Z}}_{v v}\right)
\end{array}\right)
$$

O parâmetro de rugosidade amostral $(\widehat{\alpha})$ é calculado pela média dos canais de intensidade $p \in\{h h, h v, v v\}$

$$
\widehat{\alpha}=\frac{\widehat{\alpha}_{h h}+\widehat{\alpha}_{h v}+\widehat{\alpha}_{v v}}{3},
$$

em que o estimador $\widehat{\alpha}_{p}$ é definido de acordo com a distribuição analisada:

- para o modelo $\mathcal{K}_{P}(\alpha, C, \eta)$

$$
\hat{\alpha}_{p}=\frac{{\widehat{m_{1}}}^{2}\left(\widetilde{\boldsymbol{Z}}_{p}\right)(\hat{\eta}+1)}{\hat{\eta}\left(\widehat{m}_{2}\left(\widetilde{\boldsymbol{Z}}_{p}\right)-{\widehat{m_{1}}}^{2}\left(\widetilde{\boldsymbol{Z}}_{p}\right)\right)-{\widehat{m_{1}}}^{2}\left(\widetilde{\boldsymbol{Z}}_{p}\right)}
$$

- para o modelo $\mathcal{G}_{P}^{0}(\alpha, C, \eta)$

$$
\frac{\Gamma^{2}\left(-\hat{\alpha}_{p}-1 / 4\right) \Gamma^{2}(\hat{\eta}+1 / 4)}{\Gamma\left(-\hat{\alpha}_{p}-1 / 2\right) \Gamma(\hat{\eta}+1 / 2) \Gamma\left(-\hat{\alpha}_{p}\right) \Gamma(\hat{\eta})}-\frac{{\widehat{m_{1 / 4}}}^{2}\left(\widetilde{\boldsymbol{Z}}_{p}\right)}{\widehat{m_{1 / 2}}\left(\widetilde{\boldsymbol{Z}}_{p}\right)}=0 .
$$

O próximo capítulo apresenta o método de segmentação proposto baseado nos modelos e estimadores discutidos neste capítulo. 


\section{Capítulo 4}

\section{Métodos de Segmentação de Imagens PoISAR multi-look}

Este capítulo apresenta alguns métodos de segmentação de imagens SAR polarimétricas multi-look, dando ênfase ao método de segmentação SEM proposto nesta tese.

O capítulo está dividido em três seções principais. A primeira seção apresenta uma revisão bibliográfica sobre o assunto e os métodos utilizados nas comparações com o método de segmentação proposto. A segunda seção apresenta o método SEM proposto nesta tese. A terceira seção apresenta os métodos de inicializações.

Segundo Pratt (2001), a segmentação de imagens consiste em dividir a imagem em regiões disjuntas, em que cada região possui atributos similares. O objetivo é simplificar os processamentos posteriores, como a análise ou classificação de dados, já que a imagem sofre um mapeamento de pixels em regiões. O problema da segmentação se resume em definir métodos e critérios que suportem a natureza intrínseca de cada região, como acontece com o sistema visual humano. Muitas abordagens foram definidas, como as técnicas de detecção de bordas, ajuste de regiões, crescimento de regiões, redes neurais, contornos ativos, agrupamento de dados e baseadas em métodos estatísticos (COSTA; JUNIOR, 2001; GONZALEZ; WOODS, 2002; PRATT, 2001).

Em imagens PolSAR multi-look, verificou-se que muitas pesquisas utilizam as técnicas de agrupamento de dados ou que agrupam regiões como segmentador. O problema central das técnicas de agrupamento de dados consistem em dividir a imagem em grupos (segmentos ou regiões) tais que a semelhança inter grupo deve ser máxima e a entre grupos mínima de acordo com alguma medida de proximidade (DUDA; HART; STORK, 2001). $\mathrm{Na}$ segmentação de imagens, a diferença entre os métodos depende de três fatores, que são: o tipo de inicialização, a técnica de agrupamento e a medida de proximidade. Assim, as técnicas de agrupamento com respectivas métricas são discutidas nas duas primeiras 
seções, enquanto que a última seção apresenta os tipos de inicializações utilizados nesta tese.

A próxima seção apresenta alguns métodos de segmentação de imagens PolSAR multilook.

\subsection{Trabalhos Relacionados}

Muitos métodos de segmentação aplicados a imagens PolSAR multi-look utilizam métricas obtidas com a clássica distribuição Wishart (LEE; POTTIER, 2009). No geral, as métricas foram definidas de acordo com duas abordagens: com o logaritmo natural da função densidade de probabilidade (de acordo com o conceito de máxima verossimilhança) ou com o teste da razão de verossimilhança. A distância da primeira abordagem está definida na Equação (4.4)(página 57). Há poucas pesquisas na área utilizando as distribuições de textura, como as distribuições $\mathcal{K}_{P}$ e $\mathcal{G}_{P}^{0}$. Alguns métodos estão citados nesta seção.

Em Yu e Acton (2000), as regiões iniciais são detectadas utilizando o método Watershed com o coeficiente de variação, cujos valores mais altos dos coeficientes de variação na imagem indicam a presença de bordas. Em seguida, o método agrupa as regiões de forma hierárquica utilizando a distância obtida com o logaritmo da função densidade de probabilidade da distribuição Wishart ou da distribuição $\mathcal{K}_{P}$.

Em Skriver et al. (2002), o algoritmo de segmentação MUM (Merge Using Moments) é estendido a imagens PolSAR multi-look. O algoritmo MUM é inicializado com a divisão da imagem em blocos pequenos e, em seguida, estes blocos são agrupados de forma hierárquica utilizando métricas estatísticas (OLIVER; QUEGAN, 1998). Na aplicação a imagens PolSAR multi-look, utilizou-se a distância obtida com o teste da razão de verossimilhança da distribuição Wishart.

Em Beaulieu e Touzi (2004), o método de segmentação agrupa as regiões com um critério de seleção baseado na máxima verossimilhança da distribuição Wishart ou da distribuição $\mathcal{K}_{P}$. Em Bombrun e Beaulieu (2008), este método de segmentação foi adaptado com um critério de seleção utilizando a distribuição de textura KummerU.

Em Kersten, Lee e Ainsworth (2005), há comparações entre técnicas de agrupamento de dados Fuzzy e o algoritmo EM. As técnicas de agrupamento de dados Fuzzy diferenciam no tipo de medida de dissimilaridade utilizadas, cujas métricas são as distâncias Euclidia- 
na, de Manhattan, Bartlett e uma distância obtida com a distribuição Wishart. A técnica de agrupamento de dados pelo algoritmo EM é definida com um modelo de mistura de distribuições Wishart.

Em Ayed, Mitiche e Belhadj (2006), um método variacional é proposto utilizando a distribuição Wishart e contornos ativos.

Em Cao et al. (2007), o processo de inicialização é fornecido combinando a imagem SPAN (soma dos canais de intensidade) com a técnica de decomposição de alvos $\mathrm{H} / \alpha / \mathrm{A}$ (LEE; POTTIER, 2009). Em seguida, a imagem é segmentada utilizando um método de agrupamento de dados hierárquico aglomerativo e as distâncias obtidas com o logaritmo da função densidade de probabilidade e com o teste da razão de verossimilhança da distribuição Wishart. O método também fornece o número de grupos (segmentos) ideal à imagem segmentada comparando os valores de log-verossimilhança obtidos a cada iteração. Em Lee e Pottier (2009), há uma discussão sobre os métodos de segmentação e classificação utilizando a distribuição Wishart e as inicializações por decomposição de alvos.

Em Dong, Milne e Forster (2001) e Khan, Yang e Zhang (2007), alguns atributos na matriz de covariância complexa $\eta$-look são utilizados. Khan, Yang e Zhang (2007) utilizam o algoritmo EM para calcular as estimativas dos parâmetros de um modelo de mistura que irão alimentar o método de segmentação. Neste artigo, cada pixel é definido por um vetor de atributos independentes, em que as intensidades são modeladas por uma distribuição Gamma e os dados complexos, separados em informação de amplitude e fase, são modelados pelas respectivas distribuições Rayleigh e Multilook Phase Difference. Dong, Milne e Forster (2001) propõem um método de segmentação utilizando um modelo com campos aleatórios Markovianos gaussianos.

As pesquisas aplicadas a segmentação de imagens PolSAR multi-look utilizando a distribuição $\mathcal{G}_{P}^{0}$ foram propostas nesta tese em Horta, Mascarenhas e Frery (2008), Horta et al. (2008). Em Nascimento (2008), Nascimento, Cintra e Frery (2009), oito distâncias foram derivadas com a distribuição $\mathcal{G}^{0}$ que modela as imagens SAR de intensidade. Em Gambini et al. (2006, 2008), há aplicações da distribuição $\mathcal{G}^{0}$ à segmentação de imagens SAR de amplitude. Estas pesquisas com os dados de intensidade e amplitude foram citadas nesta seção pela relevância do trabalho na aplicação à segmentação de imagens com a distribuição $\mathcal{G}^{0}$.

Dentre os métodos de segmentação propostos na literatura, dois métodos foram selecionados para comparações com a técnica de segmentação proposta nesta tese. O primeiro método de segmentação utiliza o algoritmo EM com a mistura de distribuições Wishart (KERSTEN; LEE; AINSWORTH, 2005). Este método foi selecionado para analisar a influência do passo estocástico do método SEM. 
O segundo método de segmentação utiliza o algoritmo de agrupamento de dados kmédias com a distância baseada na distribuição Wishart (CAO et al., 2007). A ferramenta educacional PolSARpro ${ }^{1}$ utiliza este método como segmentador (LEE; POTTIER, 2009).

As próximas seções descrevem o método EM e k-médias.

\subsubsection{Método de Segmentação usando o EM}

Em Kersten, Lee e Ainsworth (2005), um dos métodos de agrupamento de dados analisados é o algoritmo EM (Expectation-Mazimization) de um modelo de mistura de distribuições Wishart $\left(W(C, \eta)\right.$, ver Seção 4.2.1). Seja $\theta_{j}^{(k)}=\left(C_{j}^{(k)}, \rho_{j}^{(k)}\right)$ o conjunto paramétrico da $j$-ésima distribuição da mistura na $k$-ésima iteração e $\boldsymbol{z}_{i}$ é o atributo do $i$-ésimo pixel da imagem PolSAR de tamanho $n$. O dado $\boldsymbol{z}_{i}$ é a matriz de covariância complexa $\eta$-look (ver Equação (3.4) na página 42). O número equivalente de looks $(\widehat{\eta})$ é previamente estimado com a Equação (3.15) (página 51) e o número de distribuições $g$ é conhecido. O método de agrupamento de dados EM com a mistura de distribuições Wishart é definido por:

- Passo-E: Atualizar as probabilidades a posteriori para cada $i$-ésimo pixel e $j$-ésimo grupo, denotada por $\tau_{i j}^{(k)}$ e definida na Equação (4.6) (página 60), cuja função densidade de probabilidade da distribuição $W(C, \eta)$ é definida na Equação (3.6) (página 43);

- Passo-M: Atualizar as estimativas dos parâmetros definidas com

$$
\widehat{\rho}_{j}^{(k+1)}=\frac{\sum_{i=1}^{n} \tau_{i j}^{(k)}}{n} \text { e } \widehat{C}_{j}^{(k+1)}=\frac{\sum_{i=1}^{n}\left(\boldsymbol{z}_{i} \cdot \tau_{i j}^{(k)}\right)}{\sum_{i=1}^{n} \tau_{i j}^{(k)}} .
$$

O algoritmo repete alternadamente estes dois passos até a sua convergência. Duas formas de aferir a convergência do algoritmo EM são os critérios das Equações 2.6 e 2.7 (página 23).

Como comentado na Seção 2.4 (página 35), o algoritmo EM calcula as estimativas dos parâmetros do modelo de mistura, ou seja, define a distribuição de cada segmento da imagem. Em seguida, a segmentação é obtida de acordo com uma regra de decisão estabelecida e as estimativas calculadas no processamento anterior. Utilizou-se a regra de decisão definida na Equação (2.34) (página 35), considerando, que nos modelos de mistura de distribuições Wishart, cada grupo tem o conjunto paramétrico $\boldsymbol{\psi}_{j}=\left(C_{j}^{(k)}, \rho_{j}^{(k)}\right)$.

\footnotetext{
${ }^{1}$ A ferramenta educacional PolSARpro (Polarimetric SAR Data Processing and Educational Tool) da Agência Espacial Européia (ESA) está disponível em http://earth.esa.int/polsarpro/.
} 


\subsubsection{Método de Segmentação usando o k-médias}

Em Cao et al. (2007), utiliza-se o algoritmo de agrupamento de dados k-médias e uma distância derivada com a distribuição Wishart no método de segmentação de imagens. Esse algoritmo em conjunto com a inicialização por decomposição de alvos também é utilizado como método de segmentação na ferramenta PolSARpro (LEE; POTTIER, 2009).

A medida de dissimilaridade do algoritmo k-médias é definida tomando como base o método de máxima verossimilhança da distribuição Wishart (CAO et al., 2007; LEE; POTTIER, 2009). Considerando o logaritmo da função densidade de probabilidade da distribuição $W(C, \eta)$ (ver Equação (3.6) na página 43), obtém-se

$$
d_{s}(\boldsymbol{z}, C)=-\eta \ln (|C|)-\eta \operatorname{Tr}\left(C^{-1} \boldsymbol{z}\right)+(\eta m) \ln (\eta)+(\eta-m) \ln (|\boldsymbol{z}|)-\ln (h(\eta, m)) .
$$

Desprezando-se os termos que não dependem do parâmetro $C$, obtém-se a medida de similaridade definida por

$$
d_{s}(\boldsymbol{z}, C)=-\eta \ln (|C|)-\eta \operatorname{Tr}\left(C^{-1} \boldsymbol{z}\right)
$$

Então, pode-se definir uma medida de dissimilaridade com

$$
d(\boldsymbol{z}, C)=\frac{-1}{\eta} \cdot d_{s}(\boldsymbol{z}, C)=\ln (|C|)+\operatorname{Tr}\left(C^{-1} \boldsymbol{z}\right) .
$$

Seja $\boldsymbol{z}_{i}$ o atributo do $i$-ésimo pixel da imagem PolSAR multi-look de tamanho $n$ e $\left\{\Omega_{1}^{(0)}, \ldots, \Omega_{g}^{(0)}\right\}$ a partição inicial com $g$ grupos, o método de agrupamento de dados kmédias com a distribuição Wishart é definido por:

- Passo-1: Calcular os centróides de cada grupo com a matriz de covariância amostral (ver Equação (3.16) na página 51) de todos os pixels do grupo $\Omega_{j}^{(k)}$, denotada por $\widehat{C}_{j}^{(k)}=\widehat{m_{1}}(\tilde{\boldsymbol{Z}}), \tilde{\boldsymbol{Z}}=\left\{\left(\boldsymbol{z}_{1}, \ldots, \boldsymbol{z}_{n}\right) \mid \boldsymbol{z}_{i} \in \Omega_{j}^{(k)}\right\}$;

- Passo-2: Gerar uma partição $\left\{\Omega_{1}^{(k+1)}, \ldots, \Omega_{g}^{(k+1)}\right\}$ associando cada $i$-ésimo pixel ao grupo $\Omega_{j}^{(k+1)}$ de acordo com

$$
d\left(\boldsymbol{z}_{i}, \widehat{C}_{j}^{(k)}\right)<d\left(\boldsymbol{z}_{i}, \widehat{C}_{h}^{(k)}\right) \rightarrow \boldsymbol{z}_{i} \in \Omega_{j}^{(k+1)}, \forall i=1, \ldots, n \text { e } j, h=1, \ldots, g \text { e } j \neq h,
$$

em que $d\left(\boldsymbol{z}_{i}, \widehat{C}_{j}^{(k)}\right)$ é a distância definida na Equação (4.4).

O algoritmo repete alternadamente até a sua convergência. Como exemplo de critério de convergência, pode-se definir o erro absoluto entre os passos sucessivos dos centróides, 
denotado por $\left|\widehat{C}_{j}^{(k)}-\widehat{C}_{j}^{(k+1)}\right|$. Se o erro absoluto dos centróides for menor do que um limiar estipulado, então, considera-se que o algoritmo convergiu.

\subsection{Método de Segmentação SEM proposto}

Nesta tese, o método de segmentação proposto combina os modelos de mistura de distribuições Wishart, $\mathcal{K}_{P}$ ou $\mathcal{G}_{P}^{0}$ com o algoritmo SEM e com os estimadores obtidos pelo método dos momentos. Utilizaram-se os modelos de mistura de uma única família e, também, os modelos de um dicionário de famílias.

Em Moser, Zerubia e Serpico (2006), o algoritmo SEM é aplicado à análise de imagens SAR de amplitude, cujos atributos são modelados com a mistura de distribuições de um dicionário de famílias. Na proposta de Moser, Zerubia e Serpico (2006), os estimadores foram definidos com o método MoLC (Method of Log-Cumulants). Esse método utiliza a transformada de Mellin e momentos amostrais para obter os estimadores dos parâmetros de uma distribuição.

Nesta seção o método de segmentação proposto foi dividido em três partes: a primeira apresenta os modelos de mistura de distribuições utilizados na segmentação, a segunda apresenta o método de segmentação SEM e a terceira seção apresenta a adaptação do método de segmentação SEM ao modelo de mistura do dicionário de famílias.

\subsubsection{Modelos de Mistura de Distribuições}

Como descrito na seção 2.2.1, a mistura de distribuições é caracterizada por um número de distribuições $\boldsymbol{D}=\left(D\left(\theta_{1}\right) \ldots, D\left(\theta_{g}\right)\right)$, com respectivas densidades $f_{1}, \ldots, f_{g}$, e probabilidades $\boldsymbol{\rho}=\left(\rho_{1}, \ldots, \rho_{g}\right)$ que definem o modelo $M(\boldsymbol{\theta}, \boldsymbol{\rho})$ da Equação (2.8) (página 26).

No método proposto, os dados da imagem polarimétrica multi-look são ocorrências de variáveis aleatórias que seguem um modelo de mistura de distribuições. Assim, a matriz de covariância complexa $\eta$-look da Equação (3.4) (página 42) de cada coordenada da imagem é modelada por $\boldsymbol{Z} \sim M(\boldsymbol{\theta}, \boldsymbol{\rho})$.

O modelo de mistura pode ser composto por distribuições de uma única família, como a mistura de distribuições $W(C, \eta)$, ou por um dicionário de famílias denotado por 
$\mathcal{D}=\left\{W(C, \eta), \mathcal{K}_{P}(\alpha, C, \eta), \mathcal{G}_{P}^{0}(\alpha, C, \eta)\right\}$. Cada distribuição $D\left(\theta_{j}\right)$ do modelo corresponde a uma região específica (segmento) da imagem.

Para todos os modelos de mistura, a estimativa do número equivalente de looks $(\widehat{\eta})$ é calculada previamente e fornecida ao método de segmentação.

A mistura de distribuições Wishart contém o conjunto paramétrico definido por $\boldsymbol{\theta}=\left(C_{1}, \ldots, C_{g}\right)$. Cada distribuição $D\left(\theta_{j}\right)$ do modelo apresenta a função densidade de probabilidade $f_{\boldsymbol{Z}_{j}}\left(\boldsymbol{z} \mid C_{j}\right)$ definida na Equação (3.6) (página 43).

A mistura de distribuições $\mathcal{K}_{P}$ contém o conjunto paramétrico definido por $\boldsymbol{\theta}=$ $\left(\alpha_{1}, \ldots, \alpha_{g}, C_{1}, \ldots, C_{g}\right) \operatorname{com} \alpha>0$. Cada distribuição $D\left(\theta_{j}\right)$ do modelo apresenta a função densidade de probabilidade $f_{\boldsymbol{Z}_{j}}\left(\boldsymbol{z} \mid\left(\alpha_{j}, C_{j}\right)\right)$ definida na Equação (3.12) (página 48).

A mistura de distribuições $\mathcal{G}_{P}^{0}$ contém o conjunto paramétrico definido por $\boldsymbol{\theta}=$ $\left(\alpha_{1}, \ldots, \alpha_{g}, C_{1}, \ldots, C_{g}\right)$ com $\alpha<-1$. Cada distribuição $D\left(\theta_{j}\right)$ do modelo apresenta a função densidade de probabilidade $f_{\boldsymbol{Z}_{j}}\left(\boldsymbol{z} \mid\left(\alpha_{j}, C_{j}\right)\right)$ definida na Equação (3.13) (página 49).

Na mistura do dicionário de famílias $\mathcal{D}=\left\{W(C, \eta), \mathcal{K}_{P}(\alpha, C, \eta), \mathcal{G}_{P}^{0}(\alpha, C, \eta)\right\}$, o conjunto paramétrico é definido de acordo com as distribuições selecionadas. No caso geral, o conjunto paramétrico é definido por $\boldsymbol{\theta}=\left(\alpha_{1}, \ldots, \alpha_{g}, C_{1}, \ldots, C_{g}\right)$. Quando a distribuição Wishart é selecionada, o parâmetro $\alpha$ é desconsiderado. Por exemplo, se a $g$-ésima distribuição da mistura for da família Wishart, o conjunto paramétrico é definido por $\boldsymbol{\theta}=\left(\alpha_{1}, \ldots, \alpha_{g-1}, C_{1}, \ldots, C_{g}\right)$. A função densidade de probabilidade de cada distribuição $D\left(\theta_{j}\right)$ também é definida pela família selecionada.

Para aplicar a mistura de distribuições Wishart, $\mathcal{K}_{P}, \mathcal{G}_{P}^{0}$ e mistura do dicionário de famílias $\mathcal{D}$ à segmentação de imagens polarimétricas multi-look, utilizou-se o algoritmo SEM em conjunto com os estimadores obtidos pelo método dos momentos.

A próxima seção apresenta a técnica de segmentação proposta.

\subsubsection{Método de Segmentação SEM}

O SEM (Stochastic Expectation-Maximization) é uma versão estocástica do algoritmo EM (ver Seção 2.3 na página 32). De acordo com o tipo de distribuição utilizada no algoritmo EM, a solução analítica da maximização da esperança condicional no passo-M pode ser de difícil cômputo, como é o caso da mistura de distribuições $\mathcal{G}_{P}^{0}$ ou $\mathcal{K}_{P}$. O passo estocático do SEM torna o algoritmo flexível à maximização do passo-M, sendo possível a inclusão de estimadores obtidos pelo método dos momentos (MOSER; ZERUBIA; SER- 
PICO, 2006). Em Kersten, Lee e Ainsworth (2005), o algoritmo EM foi utilizado no cálculo das estimativas do parâmetro $\widehat{C}$ do modelo de mistura de distribuições Wishart.

A aplicação do método de agrupamento de dados SEM aos modelos de mistura de distribuições $\mathcal{G}_{P}^{0}$ ou Wishart foi proposta com o desenvolvimento desta tese em Horta, Mascarenhas e Frery (2007b). Seja $\left(\theta_{j}^{(k)}, \rho_{j}^{(k)}\right)$ o conjunto paramétrico da $j$-ésima distribuição da mistura na $k$-ésima iteração e $\boldsymbol{z}_{i}$ é o atributo do $i$-ésimo pixel da imagem PolSAR de tamanho $n$. O dado $\boldsymbol{z}_{i}$ é uma matriz de covariância complexa $\eta$-look da Equação (3.4) (página 42). O número equivalente de looks ( $\widehat{\eta})$ é previamente estimado com a Equação (3.15) (página 51) e o número de distribuições $g$ é conhecido.

O método de agrupamento de dados SEM é definido por:

- Passo-E: Atualizar as probabilidades a posteriori para cada $i$-ésimo pixel e $j$-ésimo grupo

$$
\tau_{i j}^{(k)}=\frac{\rho_{j}^{(k)} f_{\boldsymbol{Z}_{j}}\left(\boldsymbol{z}_{i} \mid \theta_{j}^{(k)}\right)}{\sum_{\ell=1}^{g} \rho_{\ell}^{(k)} f_{\boldsymbol{Z} \ell}\left(\boldsymbol{z}_{i} \mid \theta_{\ell}^{(k)}\right)},
$$

em que cada densidade é definida pela família utilizada: $W(C, \eta), \mathcal{K}_{P}(\alpha, C, \eta)$ ou $\mathcal{G}_{P}^{0}(\alpha, C, \eta)$ (ver as respectivas Equações (3.6), (3.12) e (3.13) nas páginas 43, 48 e 49$)$;

- Passo-S: Amostrar, para cada $1 \leq i \leq n$, uma realização $\omega_{i}$ em $\{1, \ldots, g\}$ e associar o $i$-ésimo pixel ao grupo em que $j=\omega_{i}$. A amostragem é realizada de acordo com a distribuição Multinomial em $g$ categorias com as probabilidades das estimativas atuais $\tau_{i j}^{(k)}$, ou seja, com o modelo $\mathcal{M}_{g}\left(1, \tau_{i j}^{(k)}\right.$ ) (ver Seção 2.3 na página 32 ). Este processo resulta na partição da imagem em $g$ grupos, definida por $\left\{\Omega_{1}^{(k)}, \ldots, \Omega_{g}^{(k)}\right\}$;

- Passo-M: Atualizar as estimativas dos parâmetros definidos por

$$
\widehat{\rho}_{j}^{(k+1)}=\frac{\# \Omega_{j}^{(k)}}{n}
$$

e $\widehat{\theta}_{j}^{(k+1)}$ é atualizado de acordo com a família utilizada $W(C, \eta)$ (ver Equação $(3.16)$ na página 51$), \mathcal{K}_{P}(\alpha, C, \eta)$ (ver Equações (3.18) e (3.16) na página 51$)$ ou $\mathcal{G}_{P}^{0}(\alpha, C, \eta)$ (ver Equações (3.19) e (3.16) na página 51) e utilizando todos os pixels do grupo $\Omega_{j}^{(k)}$, em que $n$ é o tamanho da imagem e $\# \Omega_{j}$ é a cardinalidade do grupo $\Omega_{j}$.

O algoritmo é repetido alternadamente entre estes três passos até a sua convergência. Utilizam-se critérios de erro na análise da convergência, como os critérios das Equações 2.6 e 2.7 (página 23).

O algoritmo SEM fornece as estimativas dos parâmetros por grupo, ajustando os dados de acordo com o modelo de mistura utilizado. Após a convergência do método, a imagem é segmentada usando a regra de decisão do máximo a posteriori, como comentado 
na Seção 2.4 (página 35). Em algumas situações, o método SEM não apresenta o maior valor de log-verossimilhança na iteração de convergência do método. Então, é mais efetivo segmentar a imagem na iteração de máxima log-verossimilhança (ver discussões no Capítulo 5).

Em Horta, Mascarenhas e Frery (2007b), os parâmetros iniciais do modelo de mistura devem ser informados, pois o método SEM é aplicado à classificação supervisionada com o intuito de analisar os modelos estatísticos utilizados, Wishart e $\mathcal{G}_{P}^{0}$. Adaptações ao caso não supervisionado são propostas em Horta et al. (2008) e Horta, Mascarenhas e Frery (2008). A Figura 1 ilustra método de segmentação SEM proposto.

O algoritmo SEM não supervisionado é definido por:

1. Inicialização: Calcular as estimativas dos parâmetros iniciais $\left(\widehat{\boldsymbol{\theta}}^{(0)}, \widehat{\boldsymbol{\rho}}^{(0)}\right)$ de acordo com os métodos de inicialização descritos na Seção 4.3;

2. Seleção de Amostras: Escolher $n a=n \cdot p$, com $0,25<p<1$, amostras da imagem $\left(\boldsymbol{z}_{1}, \ldots, \boldsymbol{z}_{n}\right)$ de tamanho $n$, utilizando a distribuição Uniforme no processo de amostragem. Sejam $\left(\boldsymbol{z}_{1}, \ldots, \boldsymbol{z}_{n a}\right)$ as amostras selecionadas, é mais efetivo limitar o conjunto de amostras no intervalo $\left[Q_{0,05}, Q_{0,95}\right]$ para desconsiderar a influência de possíveis outliers, cujos $Q_{0,05}$ e $Q_{0,95}$ são os respectivos quantis amostrais de ordem 0, 05 e 0,95 (HYNDMAN; FAN, 1996).

3. Método SEM: Executar o método de agrupamento de dados SEM.

O processo de seleção de amostras reduz os possíveis outliers da imagem, cujos valores podem influenciar a convergência do método. Além disso, o tamanho da amostra influencia diretamente no custo computacional. Quanto maior for o tamanho da imagem, maior será o custo computacional do método SEM.

Nas duas versões do SEM descritas, o número de distribuições $g$ é conhecido a priori. A inclusão da escolha do número de distribuições $g$ ao algoritmo SEM não foi analisada nesta tese. Em Figueiredo e Jain (2002) e McLachlan e Peel (2000), há uma revisão sobre os critérios de seleção do número de distribuições $g$ para o algoritmo EM e suas versões.

A próxima seção apresenta a adaptação do método SEM ao modelo de mistura do dicionário de famílias. 


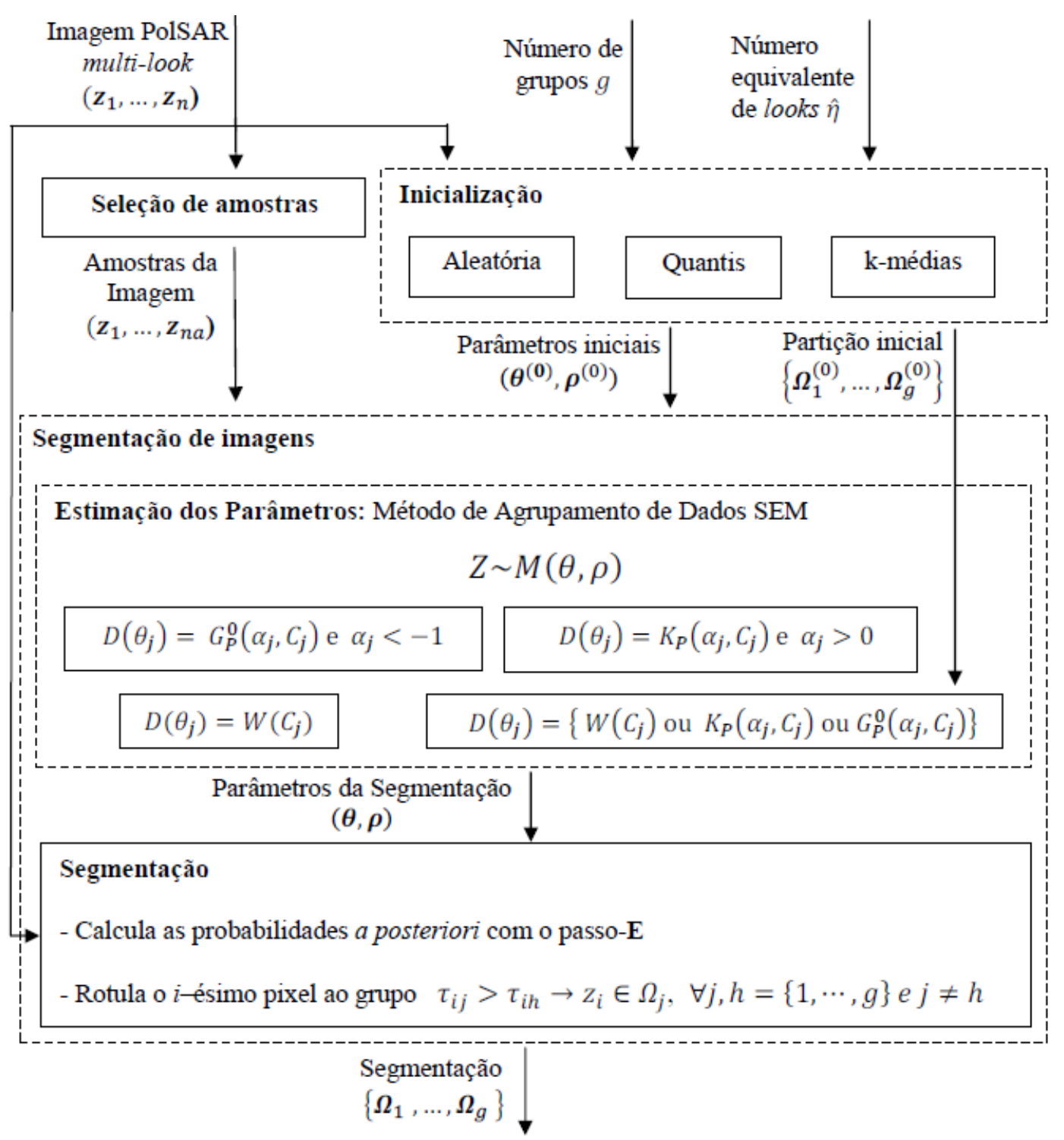

Figura 1. Método de Segmentação SEM proposto.

\subsubsection{Método de Segmentação SEM com Dicionário de Famílias}

O método de segmentação SEM proposto também foi analisado com o modelo de mistura de distribuições do dicionário de família $\mathcal{D}=\left\{W(C, \eta), \mathcal{K}_{P}(\alpha, C, \eta), \mathcal{G}_{P}^{0}(\alpha, C, \eta)\right\}$. Neste caso, o algoritmo SEM foi adaptado com a inclusão de um procedimento de seleção da família que melhor se ajusta aos dados dos grupos.

Em Moser, Zerubia e Serpico (2006), a seleção do modelo é realizada de acordo com a log-verossimilhança calculada em cada grupo, na qual a família selecionada corresponde à distribuição com maior valor de log-verossimilhança do grupo analisado. O passo de seleção do modelo foi incluído entre os passos M e E do algoritmo SEM. 
No algoritmo SEM aplicado a imagens PolSAR multi-look, o procedimento de seleção do modelo definido em Moser, Zerubia e Serpico (2006) foi introduzido no passo-E para reduzir o cálculo de operações que são custosas computacionalmente. Nas funções de densidade de probabilidade das distribuições Wishart, $\mathcal{K}_{P}$ e $\mathcal{G}_{P}^{0}$, há cálculos de inversa, determinante e multiplicação de matrizes.

Sejam $\mathcal{D}=\left\{\mathcal{D}_{1}=W, \mathcal{D}_{2}=\mathcal{K}_{P}, \mathcal{D}_{3}=\mathcal{G}_{P}^{0}\right\}$ o dicionário de famílias com respectivos parâmetros $\left\{\theta_{1 j}^{(k)}=C_{j}^{(k)}, \theta_{2 j}^{(k)}=\left(\alpha_{j}^{(k)}, C_{j}^{(k)}\right) \operatorname{com} \alpha>0, \theta_{3 j}^{(k)}=\left(\alpha_{j}^{(k)}, C_{j}^{(k)}\right) \operatorname{com} \alpha<-1\right\}$, $\left(\theta_{a j}^{(k)}, \rho_{j}^{(k)}\right)$ o conjunto paramétrico da $j$-ésima distribuição da mistura na $k$-ésima iteração e $\boldsymbol{z}_{i}$ o atributo do $i$-ésimo pixel da imagem PolSAR de tamanho $n$. O dado $\boldsymbol{z}_{i}$ é uma matriz de covariância complexa $\eta$-look da Equação (3.4) (página 42). A partição inicial é definida com $\left\{\Omega_{1}^{(0)}, \ldots, \Omega_{g}^{(0)}\right\}$ e $\left(\boldsymbol{\theta}^{(0)}, \boldsymbol{\rho}^{(0)}\right)$. O número equivalente de looks $(\widehat{\eta})$ é previamente estimado com a Equação (3.15) (página 51) e o número de distribuições $g$ é conhecido.

O algoritmo SEM com o modelo de misturas do dicionário de família é definido por:

- Passo-E:

- Passo - Seleção do modelo: Computar a log-verossimilhança para cada família $a=1, \ldots, \# \mathcal{D}$ e grupo $j=1, \ldots, g$

$$
\ell_{a j}^{(k)}(\psi)=\sum_{\boldsymbol{z} \in \Omega_{j}^{(k)}} \log f_{\boldsymbol{Z}_{j}}\left(\boldsymbol{z} \mid \theta_{a j}^{(k)}\right) .
$$

Selecionar a família $\mathcal{F}_{j}^{(k)}$ de acordo com

$$
\ell_{a j}^{(k)}(\psi)>\ell_{b j}^{(k)}(\psi) \rightarrow \mathcal{F}_{j}^{(k)}=\mathcal{D}_{a}, a \neq b, \forall a, b=1, \ldots, \# \mathcal{D} \text { e } j=1, \ldots, g
$$

- Passo - Atualização de $\tau_{i j}^{(k)}$ : Calcular as probabilidades a posteriori definidas na Equação (4.6) (página 60) para cada $i$-ésimo pixel e $j$-ésimo grupo de distribuição $\mathcal{F}_{j}^{(k)}$;

- Passo-S: Amostrar, para cada $1 \leq i \leq n$, uma realização $\omega_{i}$ em $\{1, \ldots, g\}$ e associar o $i$-ésimo pixel ao grupo em que $j=\omega_{i}$. A amostragem é realizada de acordo com a distribuição Multinomial em $g$ categorias com as probabilidades das estimativas atuais $\tau_{i j}^{(k)}$, ou seja, com o modelo $\mathcal{M}_{g}\left(1, \tau_{i j}^{(k)}\right.$ ) (ver Seção 2.3 na página 32 ). Este processo resulta na partição da imagem em $g$ grupos, definida por $\left\{\Omega_{1}^{(k)}, \ldots, \Omega_{g}^{(k)}\right\}$;

- Passo-M: Atualizar as estimativas dos parâmetros $\widehat{\rho}_{j}^{(k+1)}$ (ver a Equação (4.7) na página 60) e $\theta_{\ell j}^{(k+1)}$ da $\ell$-ésima família e $j$-ésimo grupo (ver as Equações (3.16), (3.18) e (3.19) na página 51), em que $n$ é o tamanho da imagem e $\# \Omega_{j}$ é a cardinalidade do grupo $\Omega_{j}$. 
O algoritmo é repetido alternadamente entre esses passos até a sua convergência. Utilizam-se critérios de erro na análise da convergência, como os critérios das Equações 2.6 e 2.7 (página 23). O processo de segmentação é o mesmo do método SEM com uma única família.

A próxima seção apresenta alguns métodos utilizados na inicialização dos parâmetros.

\subsection{Métodos de Inicialização}

Nos algoritmos de agrupamento de dados envolvendo mistura de distribuições e kmédias, os parâmetros iniciais $\left(\widehat{\boldsymbol{\theta}}^{(0)}=\left(\widehat{\theta}_{1}^{(0)}, \ldots, \widehat{\theta}_{g}^{(0)}\right), \widehat{\boldsymbol{\rho}}^{(0)}=\left(\widehat{\rho}_{1}^{(0)}, \ldots, \widehat{\rho}_{g}^{(0)}\right)\right)$ devem ser informados. O método de inicialização influencia significativamente na convergência do algoritmo. Em McLachlan e Peel (2000), alguns tipos de inicializações são comentadas: métodos de agrupamento de dados quando se utilizam conjuntos com grandes quantidades de dados ou pela inicialização aleatória.

Nesta tese, três tipos de inicializações são utilizadas: a aleatória, o método de agrupamento de dados k-médias e o método por quantis. Esses três tipos são definidos por:

- Inicialização Aleatória

1. Amostrar, para cada $1 \leq i \leq n$, uma realização $\omega_{i}$ em $\{1, \ldots, g\}$ e associar o $i$-ésimo pixel ao grupo em que $j=\omega_{i}$. A amostragem é realizada de acordo com a distribuição Uniforme. Este processo resulta na partição da imagem em $g$ grupos, definida por $\left\{\Omega_{1}^{(0)}, \ldots, \Omega_{g}^{(0)}\right\}$;

2. Calcular as estimativas de $\boldsymbol{\theta}^{(0)}$ com a partição $\left\{\Omega_{1}^{(0)}, \ldots, \Omega_{g}^{(0)}\right\}$ (utilizar o passoM do SEM ou o passo-1 do k-médias com Wishart). Definir as proporções equiprováveis $\left(\widehat{\boldsymbol{\rho}}^{(0)}=1 / g\right)$;

- Inicialização por k-médias

1. Gerar a imagem $S P A N=Z_{h h}+Z_{h v}+Z_{v v}$ que é a soma dos canais de intensidades da Equação (3.4) (página 42);

2. Calcular os quantis amostrais de ordem $p=0,05$ e $p=0,95$ da imagem $S P A N$, denotados por $Q_{0,05}$ e $Q_{0,95}$ (HYNDMAN; FAN, 1996). Em seguida, obter os dados da imagem $S P A N$ nesse intervalo $\left[Q_{0,05}, Q_{0,95}\right]$, ou seja, obter as amostras $S P A N_{L}=\left\{z \in S P A N \mid Q_{0,05} \leq z \leq Q_{0,95}\right\}$. O intuito é excluir os possíveis outliers; 
3. Gerar uma partição $\left\{\Omega_{1}^{(0)}, \ldots, \Omega_{g}^{(0)}\right\}$ com a imagem $S P A N_{L}$ utilizando o algoritmo de agrupamento de dados k-médias (DUDA; HART; STORK, 2001). Neste caso, a medida de dissimilaridade é a distância Euclidiana, ou seja, $d\left(z, \mu_{j}\right)=\sqrt{\left|z-\mu_{j}\right|^{2}}$, na qual $\mu_{j}$ é a média amostral do $j$-ésimo grupo;

4. Calcular as estimativas de $\boldsymbol{\theta}^{(0)}$ com a partição $\left\{\Omega_{1}^{(0)}, \ldots, \Omega_{g}^{(0)}\right\}$ (utilizar o passoM do SEM ou o passo-1 do k-médias com Wishart). Definir as proporções equiprováveis $\left(\widehat{\boldsymbol{\rho}}^{(0)}=1 / g\right)$;

- Inicialização por Quantis

1. Gerar a imagem $S P A N=Z_{h h}+Z_{h v}+Z_{v v}$;

2. Definir as médias dos grupos iniciais (centróides) de acordo com os quantis amostrais de ordem $\boldsymbol{p}=\left\{p_{1}, \ldots, p_{g}\right\}$, denotados por $\left\{Q_{p_{1}}, \ldots, Q_{p_{g}}\right\}$ (HYNDMAN; FAN, 1996). Neste caso, os centróides são definidos pela sequência $\boldsymbol{p}=(0,05+q / 2)+\{0, q, 2 \cdot q, \ldots,(g-1) \cdot q\}$, na qual $q=0,9 / g$. Os centróides pertencem ao intervalo $\left[Q_{0,05}, Q_{0,95}\right]$ para excluir possíveis outliers;

3. Gerar uma partição $\left\{\Omega_{1}^{(0)}, \ldots, \Omega_{g}^{(0)}\right\}$ associando cada pixel ao centróide mais próximo pelo erro absoluto $d_{a}\left(z, Q_{p_{j}}\right)=\left|z-Q_{p_{j}}\right|$, ou seja,

$$
d_{a}\left(z_{i}, Q_{p_{j}}\right)<d_{a}\left(z_{i}, Q_{p_{h}}\right) \rightarrow z_{i} \in \Omega_{j}^{(0)}, j \neq h, \forall j, h=1, \ldots, g \text { e } \forall z_{i} \in S P A N
$$

4. Calcular as estimativas de $\boldsymbol{\theta}^{(0)}$ com a partição $\left\{\Omega_{1}^{(0)}, \ldots, \Omega_{g}^{(0)}\right\}$ (utilizar o passoM do SEM ou o passo-1 do k-médias com Wishart). Definir proporções equiprováveis $\left(\boldsymbol{\rho}^{(0)}=1 / g\right)$;

Nas aplicações a imagens PolSAR multi-look, é crescente o número de pesquisas que utilizam a decomposição de Cloude-Pottier (ou decomposição de alvos $H / \alpha / A$ ) como método de inicialização. Alguns exemplos são os textos de Cao et al. (2007), Kersten, Lee e Ainsworth (2005) e Lee e Pottier (2009). Este método fornece três parâmetros: a entropia $H$, o ângulo $\alpha$ e o parâmetro de anisotropia $A$ que são calculados com os valores e vetores próprios da matriz de coerência. A partição inicial é obtida de acordo com os grupos formados com resultados dos parâmetros $H$ e $\alpha$ num plano bidimensional e, também, com os resultados do parâmetro A. Segundo Frery, Correia e Freitas (2007), a vantagem desse tipo de inicialização ocorre com a formação de grupos contendo diferentes características físicas em função dos mecanismos de dispersão, porém podem apresentar alvos com características que favorecem o modelo Wishart. Os métodos de inicialização por decomposição de Cloude-Pottier não foram analisados nesta tese.

Khan, Yang e Zhang (2007) utilizam o algoritmo EM com a inicialização aleatória. 
O próximo capítulo apresenta os resultados obtidos com as distribuições, os métodos de segmentação e as inicializações discutidos neste capítulo. 


\section{Capítulo 5}

\section{Resultados}

Este capítulo apresenta os resultados obtidos com duas imagens polarimétricas multilook reais. Ele está dividido em duas seções principais: a primeira apresenta as imagens PolSAR multi-look e a segunda fornece os resultados obtidos.

A segunda seção também foi dividida em quatro seções em função das análises realizadas: a primeira delas analisa a convergência do método SEM proposto com os métodos de inicialização; a segunda complementa as análises da seção anterior comparando o desempenho do método SEM com os métodos de inicialização; a terceira apresenta as comparações entre os métodos SEM, EM e k-médias; enquanto que a última fornece os resultados obtidos do método SEM com o modelo de mistura do dicionário de famílias.

A próxima seção apresenta as imagens polarimétricas multi-look reais.

\subsection{Descrição das Imagens}

As análises foram realizadas com regiões obtidas de duas imagens polarimétricas multilook reais. A primeira delas fornece uma região $(450 \times 600$ pixels $)$ de uma imagem multilook ${ }^{1}(900 \times 1024$ pixels com número nominal looks $=4)$, obtida pelo sensor AIRSAR na banda $L$ sobre a cidade de São Francisco nos Estados Unidos. A Figura 2 ilustra as imagens utilizadas nas análises: a foto aérea ${ }^{2}$, a imagem SPAN (formada com a soma dos canais de intensidade) e as imagens fornecendo as amostras de teste. A análise descritiva dos atributos da imagem São Francisco estão no Apêndice A (página 99). Na imagem de

\footnotetext{
${ }^{1}$ Disponível em:http://earth.esa.int/polsarpro

${ }^{2}$ Disponível em:http://terraserver-usa.com
} 
São Francisco, analisaram-se as segmentações obtidas com $g=3$ regiões e $g=4$ regiões.

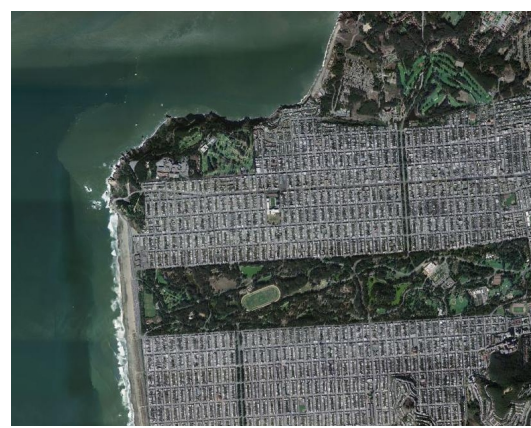

(a) Foto Aérea

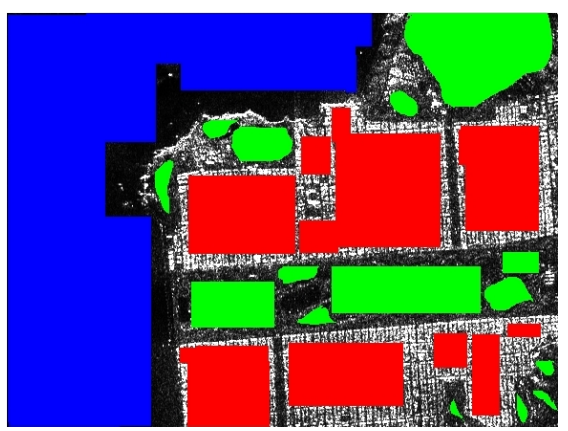

(c) Amostras de Teste com $g=3$

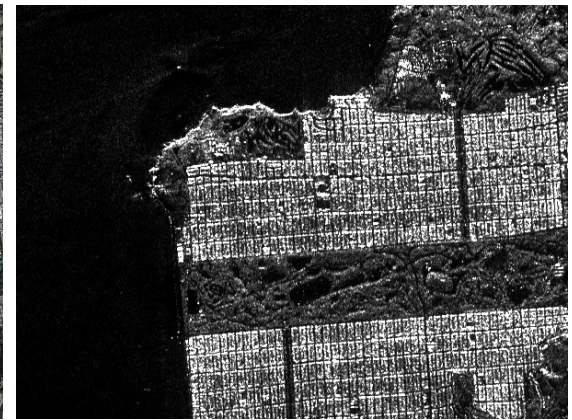

(b) $S P A N=Z_{h h}+Z_{h v}+Z_{v v}$

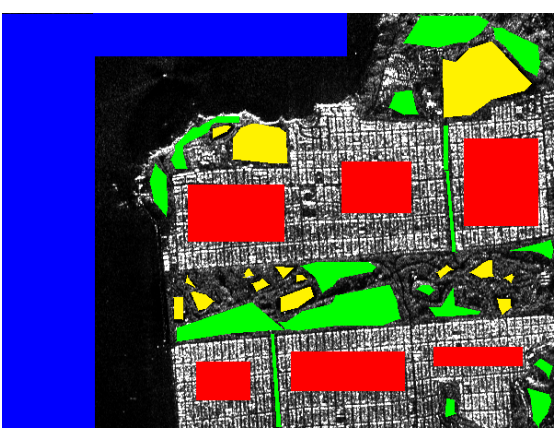

(d) Amostras de Teste com $g=4$

Figura 2. Imagens de referência da região de São Francisco.

As Tabelas 1 e 2 fornecem algumas informações das amostras de teste utilizadas. Nas amostras com 3 regiões, as áreas com florestas e gramados foram consideradas como vegetação. Nas amostras com 4 regiões, as áreas com florestas e as áreas com gramado são analisadas. Há certas amostras da região de gramado que são difíceis de separar de floresta, ocasionando uma maior heterogeneidade no grupo analisado, como mostram os parâmetros de rugosidade $(\widehat{\alpha})$ na Tabela 2 .

Tabela 1- Descrição das amostras de teste com 3 regiões da imagem São Francisco, informando as estimativas dos parâmetros, a quantidade de pixels por região $(\# \Omega)$ e a cor representativa.

\begin{tabular}{cccccccc}
\hline Regiões & $\widehat{C}_{h h}$ & $\widehat{C}_{h v}$ & $\widehat{C}_{v v}$ & $\widehat{\alpha}\left(\mathcal{G}_{P}^{0}\right)$ & $\widehat{\alpha}\left(\mathcal{K}_{P}\right)$ & $\# \Omega$ & Cor \\
\hline Oceano & $4,6 e-03$ & $6,7 e-04$ & $1,8 e-02$ & $-7,34$ & 4,08 & 90.058 & Azul \\
Vegetação & $5,8 e-02$ & $3,8 e-02$ & $6,3 e-02$ & $-2,25$ & 1,11 & 32.924 & Verde \\
Urbano & $2,5 e-01$ & $5,7 e-02$ & $2,0 e-01$ & $-1,84$ & 0,37 & 61.178 & Vermelho \\
\hline
\end{tabular}

A segunda imagem $(558 \times 491$ pixels $)$ foi obtida com o processamento multi-look (4looks) de uma região da imagem polarimétrica single-look complexa (1475 × 7134 pixels), obtida pelo sensor E_SAR na banda $L$ sobre o a cidade de Munique na Alemanha. Esta imagem foi fornecida pelo colaborador Prof. Dr. Alejandro César Frery. O processamento multi-look foi realizado no software $\mathrm{R}$ considerando o número de looks $\eta=4$. A Figura 3 
Tabela 2- Descrição das amostras de teste com 4 regiões da imagem São Francisco, informando as estimativas dos parâmetros, a quantidade de pixels por região $(\# \Omega)$ e a cor representativa.

\begin{tabular}{cccccccc}
\hline Regiões & $\widehat{C}_{h h}$ & $\widehat{C}_{h v}$ & $\widehat{C}_{v v}$ & $\widehat{\alpha}\left(\mathcal{G}_{P}^{0}\right)$ & $\widehat{\alpha}\left(\mathcal{K}_{P}\right)$ & $\# \Omega$ & Cor \\
\hline Oceano & $4,2 e-03$ & $5,9 e-04$ & $1,8 e-02$ & $-12,18$ & 8,33 & 57.650 & Azul \\
Floresta & $5,2 e-02$ & $3,6 e-02$ & $6,4 e-02$ & $-3,14$ & 1,78 & 16.137 & Verde \\
Gramado & $5,1 e-02$ & $2,8 e-02$ & $4,2 e-02$ & $-1,75$ & 1,04 & 9.380 & Amarelo \\
Urbano & $2,4 e-01$ & $5,5 e-02$ & $1,9 e-01$ & $-1,79$ & 0,35 & 27.989 & Vermelho \\
\hline
\end{tabular}

ilustra a foto aérea, a imagem $S P A N$ e a imagem com as amostras de teste. A análise descritiva dos atributos da imagem Munique estão no Apêndice A (página 99).

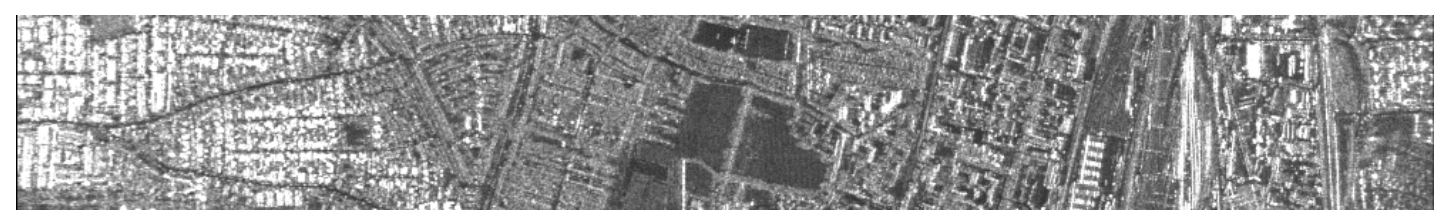

(a) Foto Aérea

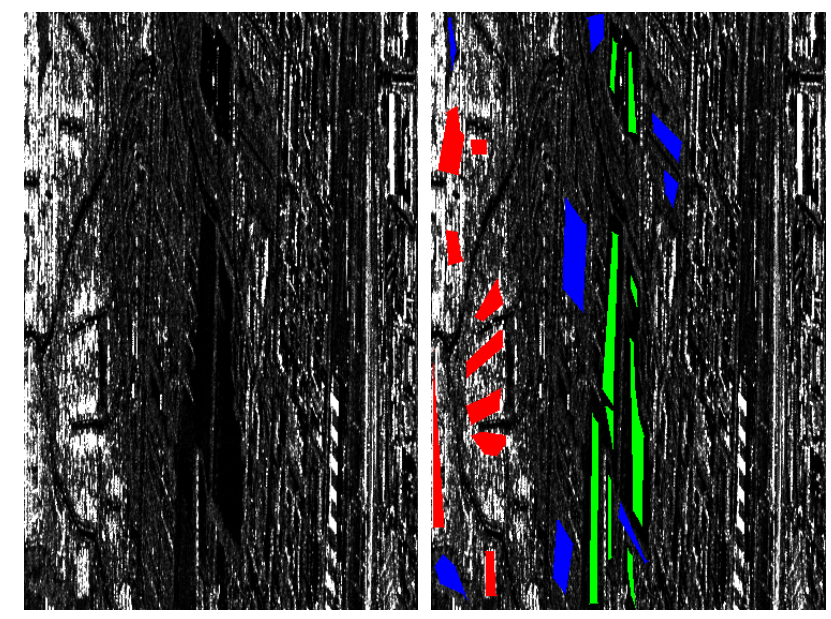

$\begin{array}{ll}\text { (b) } S P A N=Z_{h h}+Z_{h v}+Z_{v v} & \text { (c) Amostras de Teste }\end{array}$

Figura 3. Imagens de referência da região de Munique.

A Tabela 3 fornece algumas informações sobre as amostras de teste da imagem $\mathrm{Mu}-$ nique. As imagens foram segmentadas em três regiões principais: áreas mais escuras caracterizando as regiões mais homogêneas, como cultivo; áreas em cinza caracterizando as regiões heterogêneas, como vegetação, e áreas mais claras caracterizando as regiões extremamente heterogêneas, como urbano. Na Tabela 3, as estimativas dos parâmetros de rugosidade das amostras cultivo apresentaram valores com características mais heterogêneas porque diferentes tipos de cultivos foram selecionados.

As próximas seções apresentam os resultados obtidos com as imagens São Francisco e Munique. 
Tabela 3- Descrição das amostras de teste com 3 regiões da imagem Munique, informando as estimativas dos parâmetros, a quantidade de pixels por região $(\# \Omega)$ e a cor representativa.

\begin{tabular}{cccccccc}
\hline Regiões & $\widehat{C}_{h h}$ & $\widehat{C}_{h v}$ & $\widehat{C}_{v v}$ & $\widehat{\alpha}\left(\mathcal{G}_{P}^{0}\right)$ & $\widehat{\alpha}\left(\mathcal{K}_{P}\right)$ & $\# \Omega$ & Cor \\
\hline Cultivo & $1,6 e+05$ & $1,4 e+05$ & $1,6 e+04$ & $-2,34$ & 0,20 & 4.650 & Verde \\
Vegetação & $6,8 e+05$ & $5,9 e+05$ & $1,6 e+05$ & $-1,98$ & 0,56 & 4.636 & Azul \\
Urbano & $1,1 e+07$ & $7,7 e+06$ & $3,2 e+05$ & $-1,35$ & 0,33 & 4.907 & Vermelho \\
\hline
\end{tabular}

\subsection{Resultados}

Esta seção apresenta os resultados obtidos com as imagem SAR polarimétricas multilook utilizando o método de segmentação SEM proposto e os métodos de segmentação EM e k-médias.

As análises iniciais mostram as propriedades de convergência do método SEM de acordo com os modelos de mistura de distribuições Wishart, $\mathcal{K}_{P}$ e $\mathcal{G}_{P}^{0}$ utilizando diferentes métodos de inicialização. Em seguida, estes resultados são comparados através de análises quantitativas e qualitativas.

Na terceira seção, há comparações entre o método de segmentação SEM com os modelos de mistura Wishart, $\mathcal{K}_{P}$ e $\mathcal{G}_{P}^{0}$ e os dois métodos propostos na literatura, sendo estes o método EM com o modelo de mistura Wishart e o método k-médias com a medida de dissimilaridade obtida com a distribuição Wishart. A última seção apresenta os resultados obtidos com o método SEM e o modelo de mistura do dicionário de famílias $\mathcal{D}=\left\{W, \mathcal{G}_{P}^{0}, \mathcal{K}_{P}\right\}$

As medidas de avaliação adotadas foram as porcentagens de acerto e o coeficiente Kappa (CONGALTON; GREEN, 2009). Como a principal motivação deste trabalho é encontrar regiões que facilitem a classificação de imagens de Sensoriamento Remoto, estas medidas são satisfatórias. Estas medidas foram obtidas com a matriz de confusão gerada com os resultados da segmentação e as respectivas amostras de teste.

As porcentagens de acerto informam a razão entre a quantidade de pixels segmentados corretos de acordo com a amostra de teste analisada. Calcularam-se a porcentagem da amostra de teste total e as porcentagens de cada grupo da amostra de teste. Já o coeficiente Kappa compara a probabilidade de acertos contra a probabilidade de chances do evento ocorrer. O Kappa foi originalmente proposto como um método para determinar a concordância entre opiniões expressas por diferentes especialistas. No contexto da classificação, esse coeficiente determina um grau de concordância a posteriori, ou seja, dadas amostras previamente rotuladas (verdade terrestre), ele mede qual é a concordância entre esses rótulos e o resultado fornecido pelo classificador. Quanto melhor o desempenho, 
maior o valor do Kappa.

Sejam $\left\{\Omega_{1}, \ldots, \Omega_{g}\right\}$ a imagem segmentada com $g$ regiões e $\Omega^{T}=\left\{\Omega_{1}^{T}, \ldots, \Omega_{g}^{T}\right\}$ o conjunto de amostras de teste, $q_{j}^{S}=\#\left(\Omega_{j} \cap \Omega^{T}\right)$ a quantidade de amostras da $j$-ésima região da imagem segmentada que pertencem ao conjunto de amostras de teste, $q^{T}=$ $\#\left(\Omega^{T}\right)$ a quantidade total de amostras de teste, $q_{j}^{T}=\#\left(\Omega_{j}^{T}\right)$ a quantidade de amostras da $j$-ésima região de teste e $q_{i j}=\#\left(\Omega_{i} \cap \Omega_{j}^{T}\right)$ a quantidade de amostras da $i$-ésima região segmentada que pertencem à $j$-ésima região de teste. A porcentagem de acerto total é definida por $\sum_{j=1}^{g} q_{j j} / q^{T}$. A porcentagem de acerto da $j$-ésima região é definida por $q_{j j} / q_{j}^{T}$. Por fim, o coeficiente Kappa é definido pela seguinte expressão:

$$
\widehat{K}=\frac{\left[q^{T} \sum_{j=1}^{g} q_{j j}-\sum_{j=1}^{g} q_{j}^{T} q_{j}^{S}\right]}{\left[\left(q^{T}\right)^{2}-\sum_{j=1}^{g} q_{j}^{T} q_{j}^{S}\right]} .
$$

A variância do Kappa é definida por

$$
{\widehat{\sigma^{2}}}_{\widehat{K}}=\frac{1}{q^{T}}\left\{\frac{V_{1}\left(1-V_{1}\right)}{(1-V 2)^{2}}+\frac{2\left(1-V_{1}\right)\left(2 V_{1} V_{2}-V_{3}\right)}{(1-V 2)^{3}}+\frac{\left(1-V_{1}\right)^{2}\left(V_{4}-4 V_{2}^{2}\right)}{(1-V 2)^{4}}\right\},
$$

com o $V 1=\left(q^{T}\right)^{-1} \sum_{j=1}^{g} q_{j j}$, о $V 2=\left(q^{T}\right)^{-2} \sum_{j=1}^{g} q_{j}^{T} q_{j}^{S}$, о $V 3=\left(q^{T}\right)^{-2} \sum_{j=1}^{g} q_{j j}\left(q_{j}^{T}+q_{j}^{S}\right)$ е о $V 4=\left(q^{T}\right)^{-3} \sum_{i=1}^{g} \sum_{j=1}^{g} q_{i j}\left(q_{j}^{S}+q_{i}^{T}\right)^{2}$.

Algumas informações devem ser fornecidas aos método SEM, EM e k-médias. Para os três métodos, o número de distribuições da mistura $g$ é fornecido. No geral, adotaram-se $g=3$ regiões. Na imagem São Francisco, a segmentação também foi analisada com $g=4$ regiões.

O número equivalente de looks também deve ser fornecido aos métodos SEM e EM. Considerou-se o mesmo número equivalente de looks $(\widehat{\eta})$ em toda a imagem, cujas estimativas foram calculadas com a Equação 3.15 (página 51) usando dados de regiões homogêneas. Na imagem São Francisco, utilizou-se o número equivalente de looks $\widehat{\eta}=3,44$. Na imagem Munique, utilizou-se o número equivalente de looks $\widehat{\eta}=3,33$.

A complexidade do algoritmo não foi levada em consideração, mas há alguns comentários sobre o custo computacional. Os métodos de segmentação foram processados com o software estatístico $R$. Como exemplo, a Tabela 4 fornece os tempos de execução da aplicação dos métodos de segmentação na imagem São Francisco (450 × 600 pixels) com $g=4$ regiões. O computador utilizado tem as configurações: Intel Core 2 Duo T7500 de $2.2 G H z$ e com $2 G B$ de RAM.

A próxima seção apresenta as curvas de convergência dos parâmetros e log-verossimilhança de alguns resultados obtidos com o método SEM e os modelos de mistura das distribuições $W, \mathcal{K}_{P}$ e $\mathcal{G}_{P}^{0}$. 
Tabela 4- Custo computacional da aplicação dos métodos de segmentação na imagem São Francisco com 4 regiões. O determinante da matriz de covariância em cada pixel $(|\boldsymbol{Z}|)$ é calculado previamente e fornecido aos métodos SEM e EM.

\begin{tabular}{cccc}
\hline Método & $\begin{array}{c}\text { Custo de 32 iterações } \\
\text { (minutos) }\end{array}$ & $\begin{array}{c}\text { Custo de calcular }|\boldsymbol{Z}| \\
\text { (minutos) }\end{array}$ & $\begin{array}{c}\text { Total } \\
\text { (minutos) }\end{array}$ \\
\hline SEM $\mathcal{D}$ & 69 & 4 & 73 \\
SEM $\mathcal{G}_{P}^{0}$ & 63 & 4 & 67 \\
SEM $\mathcal{K}_{P}$ & 60 & 4 & 64 \\
SEM $W$ & 60 & 4 & 64 \\
EM $W$ & 57 & 4 & 61 \\
k-médias $W$ & 59 & - & 59 \\
\hline
\end{tabular}

\subsubsection{Convergência do Método SEM}

Esta seção apresenta alguns resultados de convergência dos modelos de mistura de distribuições $W, \mathcal{K}_{P}$ e $\mathcal{G}_{P}^{0}$ e a influência da utilização destes modelos no método SEM.

Em todos os resultados, o método SEM foi executado com 32 iterações. Analisaramse os resultados obtidos com as inicializações aleatória, quantil e k-médias (distância Euclidiana) na imagem São Francisco com $g=3$ regiões e, também, com o método de inicialização quantil na imagem Munique com $g=3$ regiões.

\subsubsection{Convergência com a Imagem São Francisco}

A Figura 4 ilustra as curvas de convergência da log-verossimilhança com os três regiões obtidos utilizando as inicializações quantil, aleatória e k-médias (distância Euclidiana). As curvas de convergência das estimativas dos parâmetros e as imagens segmentadas da imagem São Francisco estão ilustradas no Apêndice B (página 103).

Nos resultados obtidos com o modelo de mistura de distribuições Wishart, a log-verossimilhança converge e é crescente (ver as Figuras 4(a), 4(b) e 4(c)). Em todos os resultados, a log-verossimilhança e as estimativas dos parâmetros $(\widehat{\boldsymbol{C}}, \widehat{\boldsymbol{\rho}})$ estabilizam antes do número máximo de iterações (32) (ver as Figuras 17, 19 e 21 nas páginas 104, 106 e 108). Neste exemplo, o estimador do método dos momentos para a matriz de covariância $\widehat{C}$ garantiu que a propriedade da log-verossimilhança fosse satisfeita.

Nos resultados obtidos com o modelo de mistura de distribuições $\mathcal{K}_{P}$ utilizando os métodos de inicialização quantil ou k-médias (distância Euclidiana), a log-verossimilhança 


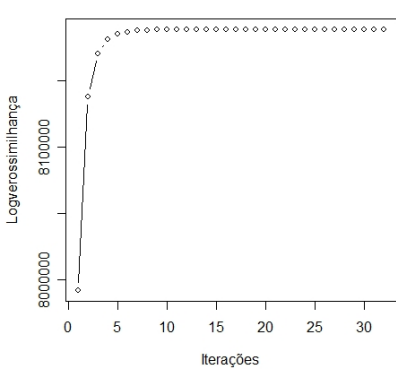

(a) $W$ : Quantil

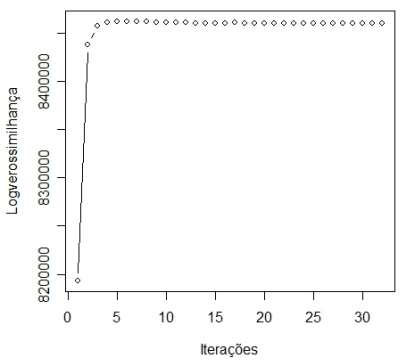

(d) $\mathcal{K}_{P}:$ Quantil

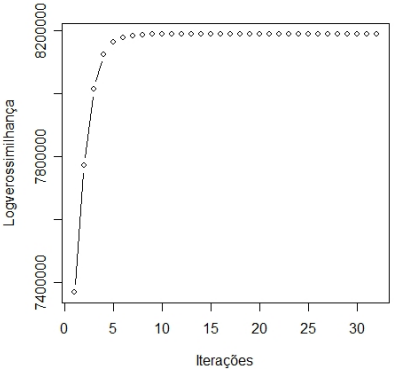

(b) $W$ : k-médias

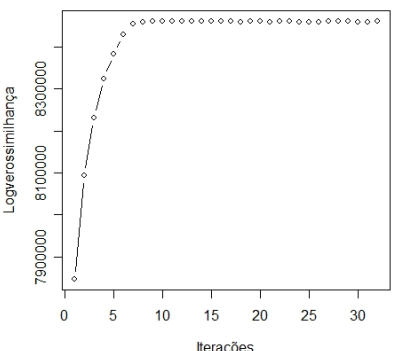

(e) $\mathcal{K}_{P}:$ k-médias

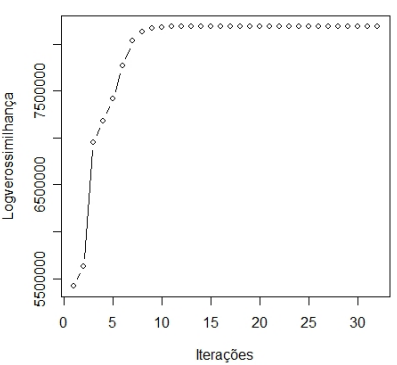

(c) $W$ : Aleatória

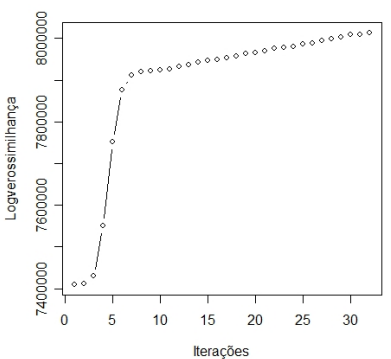

(f) $\mathcal{K}_{P}$ : Aleatória

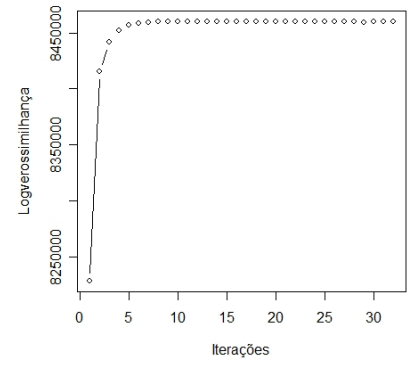

(g) $\mathcal{G}_{P}^{0}$ : Quantil

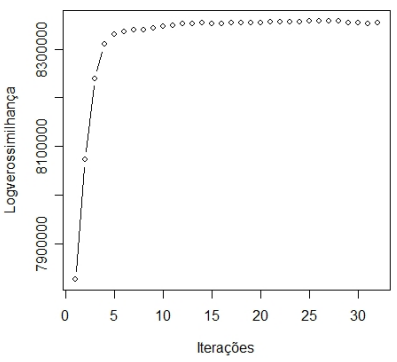

(h) $\mathcal{G}_{P}^{0}:$ k-médias

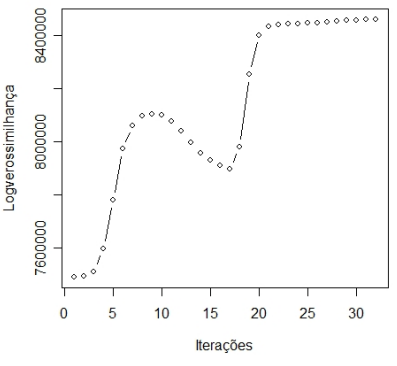

(i) $\mathcal{G}_{P}^{0}$ : Aleatória

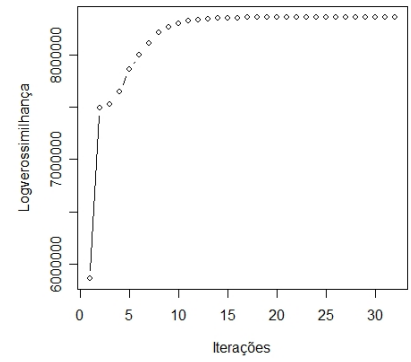

(j) $\mathcal{G}_{P}^{0}\left(\widehat{\alpha}^{(0)}=-50\right)$ : Aleatória

Figura 4. Curvas de convergência da log-verossimilhança $\ell(\widehat{\psi})$ dos resultados do método SEM aplicado a imagem de São Francisco com $g=3$ regiões e diferentes inicializações.

converge para os maiores valores, mas a propriedade de convergência não é satisfeita $\left(\ell\left(\widehat{\psi}^{(k+1)}\right) \geq \ell\left(\widehat{\psi}^{(k)}\right)\right)$ (ver as Figuras $4(\mathrm{~d})$ e $\left.4(\mathrm{e})\right)$. O estimador do parâmetro de rugosidade $\widehat{\alpha}$, obtido pelo método dos momentos, pode ter influenciado esta situação. Mesmo assim, a log-verossimilhança e as estimativas dos parâmetros da mistura $(\widehat{\boldsymbol{C}}, \widehat{\boldsymbol{\alpha}}, \widehat{\boldsymbol{\rho}})$ convergem antes de 32 iterações (ver Figuras 19 e 21 nas páginas 106 e 108). No caso da inicialização aleatória (Figura 4(f)), a log-verossimilhança não congerve até o número máximo de iterações.

Nos resultados obtidos com o modelo de mistura de distribuições $\mathcal{G}_{P}^{0}$ utilizando os métodos de inicialização quantil ou k-médias (distância Euclidiana), a log-verossimilhança converge para os maiores valores, mas a propriedade de convergência também não é satisfeita (ver as Figuras 4(g) e 4(h)). Contudo, a log-verossimilhança e os parâmetros da mistura $(\widehat{\boldsymbol{C}}, \widehat{\boldsymbol{\alpha}}, \widehat{\boldsymbol{\rho}})$ estabilizam antes de 32 iterações (ver Figuras 19 e 21). No caso da inicialização aleatória (Figura 4(i)), os resultados da log-verossimilhança são muito instáveis, apresentando um decaimento na curva. Porém, o maior valor da log-verossimilhança foi 
obtido no número máximo de iterações (32).

As inicializações aleatórias exigem mais iterações por causa de sua natureza estocástica. Na inicialização aleatória obtida, algumas condições foram especificadas: utilizou-se a distribuição Uniforme no processo de amostragem, considerando um número mínimo de amostras por região para não formar regiões muito pequenos. Estas condições atenuaram a influência do processo aleatório sobre as estimativas da matriz de covariância amostral $\widehat{C}$.

Os parâmetros de rugosidade das distribuições $\mathcal{K}_{P}$ e $\mathcal{G}_{P}^{0}$ são influenciados pela inicialização aleatória, como mostram as Figuras 17 e 18 (página 104). Quando todos os valores $\left|\widehat{\alpha}_{1}^{(0)}\right|, \ldots,\left|\widehat{\alpha}_{g}^{(0)}\right|$ são baixos, próximos de 0 ou 1 para as respectivas distribuições $\mathcal{K}_{P}$ e $\mathcal{G}_{P}^{0}$, o método de segmentação necessita de muitas iterações. No caso, todas as distribuições $D_{1}, \ldots, D_{g}$ apresentam características muitos heterogêneas, o que dificulta a convergência dos parâmetros.

Este problema pode ser contornado com a própria flexibilidade presente nos parâmetros de rugosidade. Ao atribuir valores altos a $\left|\widehat{\alpha}_{1}^{(0)}\right|, \ldots,\left|\widehat{\alpha}_{g}^{(0)}\right|$, as distribuições $\mathcal{K}_{P}$ e $\mathcal{G}_{P}^{0}$ tendem à distribuição $W$. Esta característica reduz a influência do parâmetro de rugosidade nas primeiras iterações, além de tornar o método SEM flexível a mudanças entre as distribuições $W$ e $\mathcal{K}_{P}$ ou $W$ e $\mathcal{G}_{P}^{0}$. Por exemplo, nas primeiras 5 iterações, as estimativas da rugosidade podem ser especificadas com valores muito altos $\left|\widehat{\alpha}_{j}^{(1: 5)}\right| \rightarrow \infty$, o que caracteriza o ajuste dos dados com a distribuição Wishart. Nas iterações seguintes, as estimativas do parâmetro $\left|\widehat{\alpha}_{j}^{(6: k)}\right|$ são calculadas no método SEM.

Para mostrar essa influência das estimativas iniciais da rugosidade no desempenho do método SEM, estipulou-se a rugosidade inicial com $\widehat{\alpha}_{i}^{(0)}=-50$ em cada região da mistura de distribuições $\mathcal{G}_{P}^{0}$. As estimativas da matriz de covariância amostral $\widehat{C}_{i}^{(0)}$ foram calculadas com a inicialização aleatória. A curva de convergência da log-verossimilhança $\mathcal{G}_{P}^{0}\left(\widehat{\alpha}^{(0)}=-50\right)$ está ilustrada na Figura $4(\mathrm{j})$. As curvas de convergência dos parâmetros estão ilustradas na Figura 17 (página 104). Neste exemplo, o método convergiu antes do número máximo de iterações.

\subsubsection{Convergência com a Imagem Munique}

A Figura 5 ilustra as curvas de convergência da log-verossimilhança e as estimativas do parâmetro de intensidade $\widehat{\boldsymbol{C}}_{h h}$ com os três regiões obtidos utilizando a inicialização quantil e os métodos SEM e EM. As curvas de convergência das estimativas dos parâmetros e as 
imagens segmentadas da imagem Munique estão ilustradas no Apêndice C (página 111).

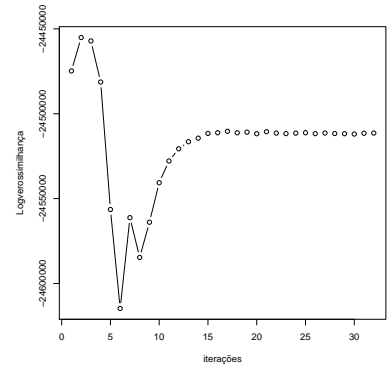

(a) SEM $\mathcal{G}_{P}^{0}: \ell(\widehat{\psi})$

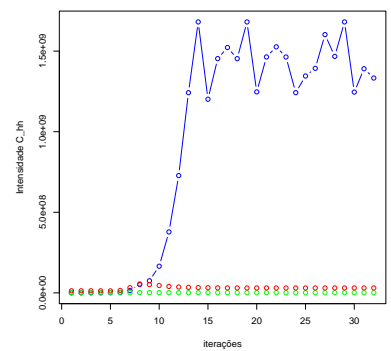

(e) SEM $\mathcal{G}_{P}^{0}: \widehat{\boldsymbol{C}}_{h h}$

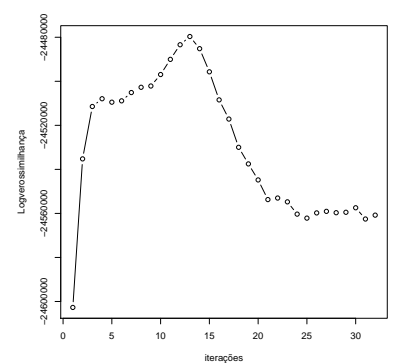

(b) $\operatorname{SEM} \mathcal{K}_{P}: \ell(\widehat{\psi})$

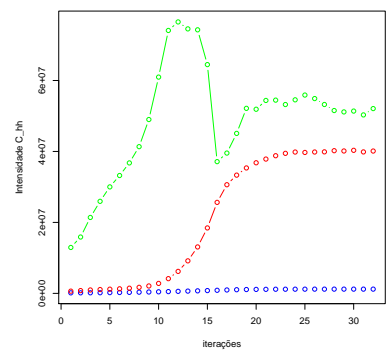

(f) $\operatorname{SEM} \mathcal{K}_{P}: \widehat{C}_{h h}$

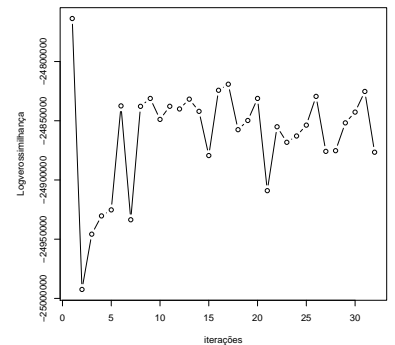

(c) $\operatorname{SEM} W: \ell(\widehat{\psi})$

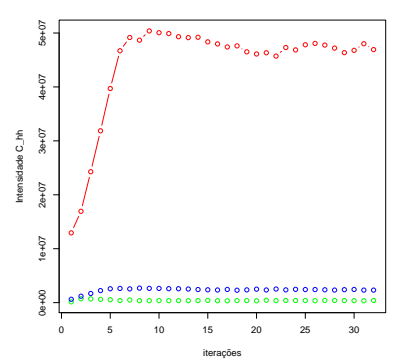

(g) SEM $W: \widehat{C}_{h h}$

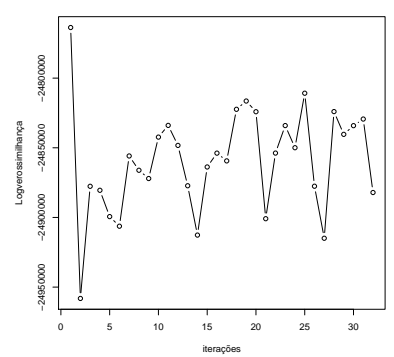

(d) $\operatorname{EM} W: \ell(\widehat{\psi})$

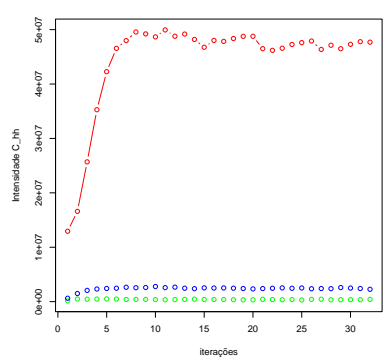

(h) EM $W: \widehat{\boldsymbol{C}}_{h h}$

Figura 5. Curvas de convergência da log-verossimilhança $\ell(\widehat{\psi})$ e da intensidade de $\widehat{C}$ na polarização $h h\left(\widehat{\boldsymbol{C}}_{h h}\right)$ dos resultados do método SEM e EM aplicados à imagem Munique com $g=3$ regiões e método de inicialização quantil. As Figuras (a-d) ilustram as curvas de convergência da log-verossimilhança. As Figuras (e-h) ilustram as curvas de convergência da intensidade amostral com as três regiões (cultivo verde, vegetação - azul e urbano - vermelho).

Com os resultados da imagem Munique, verificou-se a influência de outliers no método SEM. Os atributos desta imagem apresentam uma variabilidade muito grande e contêm alguns valores discrepantes (ver as estatísticas dos atributos da imagem no Apêndice A na página 99). Por exemplo, a média dos atributos da imagem $S P A N$ é maior que o terceiro quartil, que são: média $(6.7 e+06)$, o terceiro quartil $(2.7 e+06)$ (ver Figura 16 na página 101). Esta dispersão dos dados torna as regiões de cultivo e vegetação muito próximas quando comparadas com a área urbana, o que influencia o desempenho do método SEM com os diferentes modelos de mistura.

As Figuras 5(a), 5(b) e 5(c) ilustram a curvas de convergência da log-verossimilhança do método SEM com os diferentes modelos de mistura. Na mistura de distribuições Wishart, as log-verossimilhanças dos métodos SEM (Figura 5(c)) e EM (Figura 5(d)) apresentaram características parecidas.

Ao analisar as curvas de convergência do parâmetro $\widehat{\boldsymbol{C}}_{h h}$ na Figura 5 , verificou-se que o método SEM tendeu a definir uma região contendo os outliers (valores discrepantes). O desempenho do método a cada mistura de distribuição e, respectivas imagens segmentadas, 
dependeu do tipo de distribuição utilizada na mistura. No caso da distribuição Wishart, os resultados foram obtidos de acordo com as estimativas do parâmetro $\widehat{\boldsymbol{C}}$. Nas distribuições de textura, $\mathcal{G}_{P}^{0}$ e $\mathcal{K}_{P}$, os resultados também são influenciados pelos parâmetros de rugosidade. As curvas de convergência do conjunto paramétrico do modelo de mistura estão ilustradas na Figura 24 (página 112) e as imagens segmentadas estão ilustrados na Figura 25 (página 113).

No modelo de mistura de distribuições $\mathcal{G}_{P}^{0}$, as Figuras $5(\mathrm{e})$ e $5($ a) caracterizam bem esses resultados. Nas iterações iniciais, o decaimento da log-verossimilhança ressalta a mudança das estimativas $\widehat{\boldsymbol{C}}_{h h}$ da região azul. Quando os outliers são detectados na região azul (entre as iterações 10 e 15), a log-verossimilhança começa a convergir. Neste caso, o maior valor da log-verossimilhança indica a melhor segmentação a ser obtida entre todas as iterações.

Com a redução dos outliers no passo de seleção de amostras do método SEM, o desempenho do método melhora significativamente. A Figura 6 ilustra as curvas de convergência da log-verossimilhança com $90 \%$ do tamanho da imagem, a seleção dos pixels foi definida de acordo com os dados da imagem $S P A N$ que pertencem ao intervalo $\left[Q_{0,05}, Q_{0,95}\right]$. As curvas de convergência dos conjuntos paramétricos dos modelos de mistura $W, \mathcal{K}_{P}$ e $\mathcal{G}_{P}^{0}$ estão ilustradas na Figura 26 (página 114) e as imagens segmentadas estão ilustrados na Figura 27 (página 115).

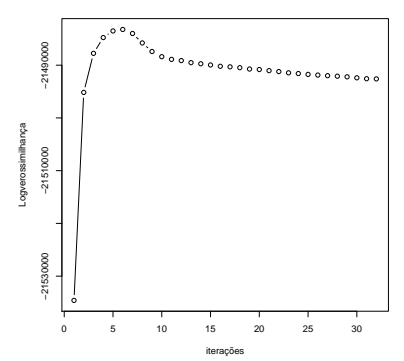

(a) $\operatorname{SEM} \mathcal{G}_{P}^{0}: \ell(\widehat{\psi})$

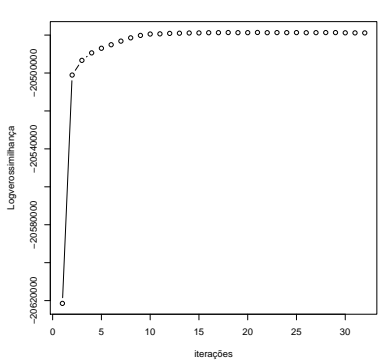

(b) $\operatorname{SEM} \mathcal{K}_{P}: \ell(\widehat{\psi})$

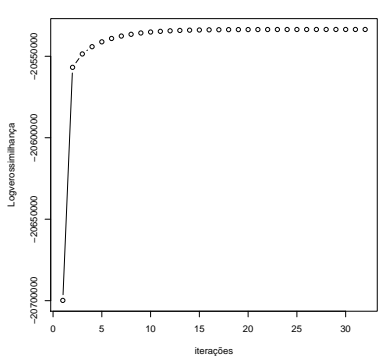

(c) $\operatorname{SEM} W: \ell(\widehat{\psi})$

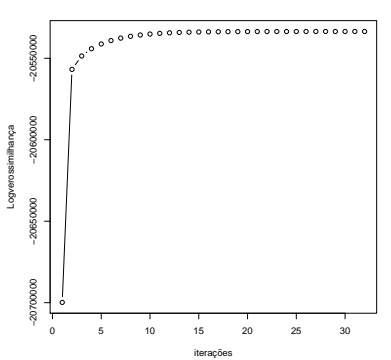

(d) EM $W: \ell(\widehat{\psi})$

Figura 6. Curvas de convergência da log-verossimilhança $\ell(\widehat{\psi})$ dos resultados do método SEM e EM aplicado a imagem Munique parcial com $g=3$ regiões e método de inicialização quantil. Os métodos SEM e EM foram processados com $90 \%$ dos pixels da imagem, consideraram-se os pixels da imagem com $S P A N \in\left[Q_{0,05}, Q_{0,95}\right]$.

Nas imagens de São Francisco e Munique, o método SEM apresenta bom desempenho com a redução dos outliers pelo quantil $Q_{0,95}$. Porém, este critério é muito restrito e não é aplicado a todas as imagem PolSAR multi-look e a todos os modelos de mistura. Mesmo com a redução dos outliers com o quantil $Q_{0,95}$, há casos em que a log-verossimilhança não é crescente, como nas Figuras 5 e 6(a). Então, para garantir que o método SEM apresente os melhores resultados a qualquer imagem PolSAR multi-look e modelo de mistura, é mais efetivo que a segmentação seja obtida na iteração que tiver o maior valor 
de log-verossimilhança, mesmo que o método convirja.

A próxima seção apresenta a comparação entre os métodos de inicialização utilizando o método de segmentação SEM.

\subsubsection{Diferentes Inicializações com o Método SEM}

Esta seção apresenta os resultados quantitativos do método SEM utilizando três tipos de inicializações: a aleatória, a técnica clássica de agrupamento de dados k-médias (distância Euclidiana) e pelos quantis amostrais (ver a Seção 4.3 na página 64). As análises foram realizadas com a imagem São Francisco, definindo $g=3$ regiões e considerando todos os pixels da imagem. A Figura 7 ilustra as partições iniciais das imagens obtidas com os três métodos de inicialização.

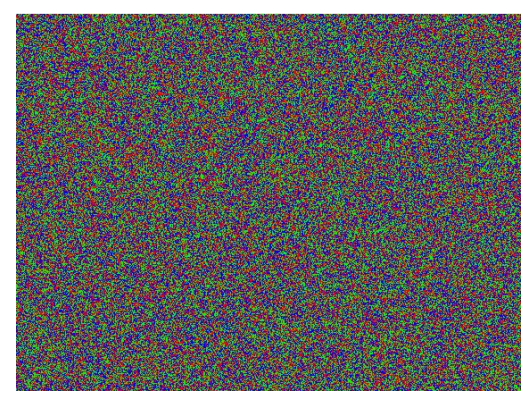

(a) Aleatória

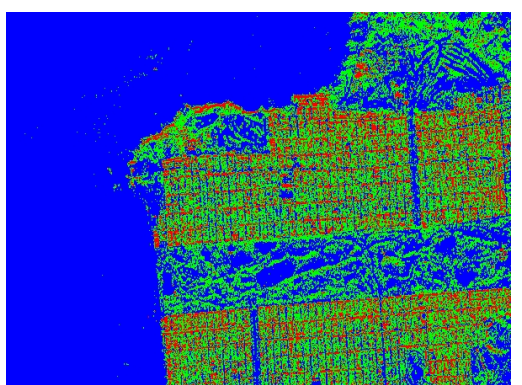

(b) k-médias

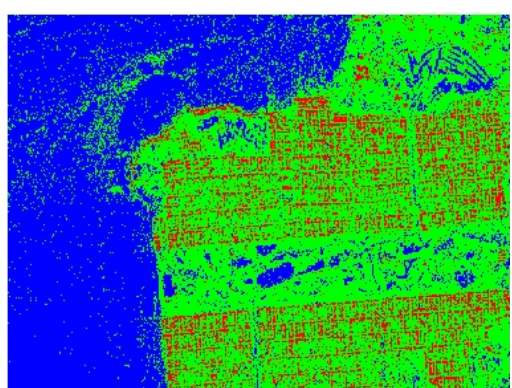

(c) Quantil

Figura 7. Diferentes inicializações da imagem São Francisco contendo $g=3$ regiões.

Em todos os resultados, o método SEM foi executado com 32 iterações. Porém, os resultados quantitativos foram obtidos na iteração em que o método SEM convergiu. O critério de convergência utilizado foi o erro absoluto relativo entre os passos sucessivos dos estimadores (ver Equação (2.7) da página 23). As Figuras 17, 19 e 21 (páginas 104, 106 e 108) mostram as curvas de convergência de alguns parâmetros dos modelos de mistura de distribuições $W(C, \eta), \mathcal{K}_{P}(\alpha, C, \eta)$ e $\mathcal{G}_{P}^{0}(\alpha, C, \eta)$. As Figuras 18, 20 e 22 (páginas 105, 107 e 109) ilustram os resultados obtidos em diferentes iterações.

A convergência do método SEM é influenciada pelo tipo de inicialização utilizada. Quando as inicializações possuem partições com grupos bem separados e próximos da segmentação desejada, o método pode convergir com poucas iterações e fornecer bons resultados. Dentre as três inicializações obtidas na Figura 7, a partição fornecida com os quantis amostrais apresentou as regiões mais representativas, sendo até melhor que o método k-médias (distância Euclidiana). 
As distribuições $W, \mathcal{K}_{P}$ e $\mathcal{G}_{P}^{0}$ possuem funções densidade de probabilidade envolvendo cálculos muito custosos computacionalmente, como o determinante e a inversa da matriz de covariância. Com isto, o método de segmentação proposto possui um custo computacional significativo a cada iteração. Na imagem de São Francisco $(450 \times 600$ pixels $)$ com $g=3$ regiões, o tempo de execução médio do método SEM foi de um minuto por iteração. Neste contexto, um método de inicialização eficaz deve ser capaz auxiliar o método de segmentação a fornecer bons resultados com poucas iterações.

A Tabela 5 mostra as porcentagens de acerto e o coeficiente Kappa de acordo com a iteração em que o método convergiu e utilizando as amostras de teste da Figura 2(c) (página 68). De acordo com o Kappa e respectivas variâncias $\left(\widehat{\sigma^{2}} \widehat{K}\right)$, o método SEM apresenta resultados semelhantes entre as segmentações com a mistura da mesma família (ver as Figuras 18, 20 e 22 nas respectivas páginas 105, 107 e 109). A diferença entre os resultados se concentra no número de iterações necessário até a convergência do método.

Tabela 5- Resultados quantitativos utilizando o método SEM e diferentes inicializações da imagem São Francisco contendo $g=3$ regiões. As porcentagem de acertos (P.A.), coeficiente Kappa $(\widehat{K})$ e variância do Kappa $\left(\widehat{\sigma^{2}} \widehat{K}\right)$ foram obtidos na iteração de convergência do método (Iter.).

\begin{tabular}{ccccccccc}
\hline $\begin{array}{c}\text { Tipos de } \\
\text { Inicial. }\end{array}$ & $\begin{array}{c}\text { Tipos de } \\
\text { Distrib. }\end{array}$ & $\begin{array}{c}\text { P.A.(\%) } \\
\text { Oceano }\end{array}$ & $\begin{array}{c}\text { P.A.(\%) } \\
\text { Vegetação }\end{array}$ & $\begin{array}{c}\text { P.A.(\%) } \\
\text { Urbano }\end{array}$ & $\begin{array}{c}\text { P.A.(\%) } \\
\text { Total }\end{array}$ & $\widehat{K}$ & $\begin{array}{c}\sigma^{2} \widehat{K} \\
(e-06)\end{array}$ & Iter. \\
\hline \multirow{4}{*}{ Aleatória } & $W$ & 96,95 & 88,11 & 45,19 & 78,17 & 0,66 & 1,73 & 17 \\
& $\mathcal{K}_{P}$ & 98,01 & 96,45 & 25,25 & 73,56 & 0,59 & 1,69 & 32 \\
& $\mathcal{G}_{P}^{0}$ & 96,68 & $\mathbf{9 7 , 5 6}$ & $\mathbf{4 8 , 9 5}$ & $\mathbf{8 0 , 9 8}$ & $\mathbf{0 , 7 1}$ & 1,60 & 32 \\
& $\mathcal{G}_{P}^{0 *}$ & $\mathbf{9 9 , 2 0}$ & 95,22 & 44,55 & $\mathbf{8 0 , 3 4}$ & 0,69 & 1,63 & 22 \\
& $*\left(\widehat{\boldsymbol{\alpha}}^{(0)}=-50\right)$ & & & & & & & \\
\hline \multirow{3}{*}{ k-médias } & $W$ & 96,92 & 87,98 & 45,41 & 78,21 & 0,66 & 1,73 & 14 \\
& $\mathcal{K}_{P}$ & 94,77 & $\mathbf{9 8 , 7 7}$ & 28,59 & 73,50 & 0,60 & 1,64 & 13 \\
& $\mathcal{G}_{P}^{0}$ & $\mathbf{9 9 , 2 8}$ & 94,73 & $\mathbf{4 6 , 2 6}$ & $\mathbf{8 0 , 8 5}$ & $\mathbf{0 , 7 0}$ & 1,62 & 11 \\
\hline \multirow{3}{*}{ Quantil } & $W$ & $\mathbf{9 6 , 9 1}$ & 88,31 & 44,90 & 78,11 & 0,66 & 1,73 & 10 \\
& $\mathcal{K}_{P}$ & $\mathbf{9 4 , 7 7}$ & $\mathbf{9 8 , 8 1}$ & 28,34 & 73,43 & 0,60 & 1,63 & 14 \\
& $\mathcal{G}_{P}^{0}$ & $\mathbf{9 6 , 7 6}$ & 97,46 & $\mathbf{5 1 , 0 1}$ & $\mathbf{8 1 , 6 9}$ & $\mathbf{0 , 7 2}$ & 1,58 & 08 \\
\hline
\end{tabular}

Como comentado na seção anterior, os parâmetros dos modelos de mistura $\mathcal{K}_{P}$ e $\mathcal{G}_{P}^{0}$ são influenciados pela inicialização aleatória, mas este processo pode ser atenuado inicializando as estimativas da rugosidade com $|\widehat{\alpha}| \rightarrow \infty$. Como exemplo, no modelo de mistura de distribuições $\mathcal{G}_{P}^{0}$, as estimativas iniciais da rugosidade foram definidas com $\left\{\widehat{\alpha}_{1}^{(0)}, \ldots, \widehat{\alpha}_{g}^{(0)}\right\}=-50$. Neste caso, o método SEM convergiu com 22 iterações.

Dentre os resultados obtidos com os diferentes métodos de inicialização, o método SEM com a inicialização por quantil forneceu bons resultados com poucas iterações. A segmentação obtida com o modelo de mistura de distribuições $\mathcal{G}_{P}^{0}$ apresentou o maior Kappa com apenas 8 iterações. A Figura 8 ilustra as segmentações do método SEM e 
inicialização quantil na iteração em que o método convergiu. Os modelos de mistura de distribuições $\mathcal{G}_{P}^{0}$ e $\mathcal{K}_{P}$ descrevem bem a região de vegetação, enquanto que o modelo com a distribuição $W$ mistura as regiões de vegetação e urbano. A distribuição $\mathcal{K}_{P}$ identifica pouco as áreas urbanas.

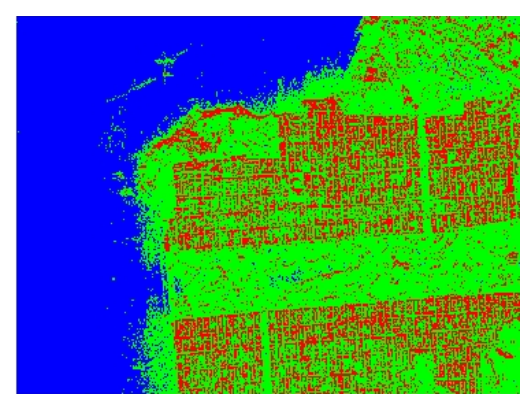

(a) $W$ : iteração 10

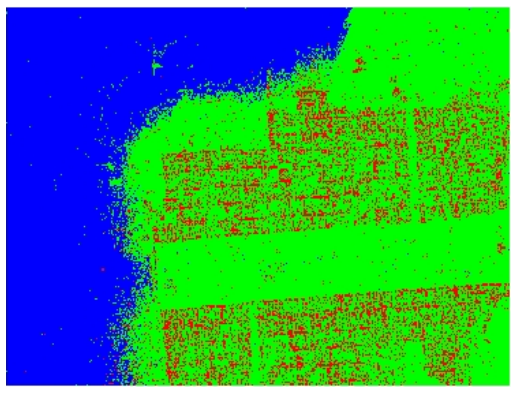

(b) $\mathcal{K}_{P}$ : iteração 14

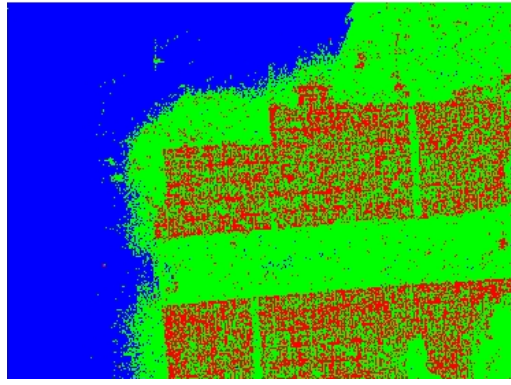

(c) $\mathcal{G}_{P}^{0}$ : iteração 8

Figura 8. Segmentações da imagem São Francisco obtidas na iteração de convergência com a inicialização quantil e $g=3$.

Os resultados obtidos com o método SEM do modelo de mistura $\mathcal{G}_{P}^{0}$ utilizando as inicializações quantil e aleatória foram publicados em Horta, Mascarenhas e Frery (2008). Em Horta, Mascarenhas e Frery (2007a, 2007b) há uma comparação entre as distribuições $W$ e $\mathcal{G}_{P}^{0}$ quando aplicados à classificação de dados.

\subsubsection{Comparações entre os Métodos de Segmentação}

Nesta seção, o método SEM com os diferentes modelos de mistura é comparado com os métodos EM e k-médias. O método EM calcula as estimativas dos parâmetros da mistura de distribuições Wishart, enquanto o método k-médias possui a medida de dissimilaridade baseada na distribuição Wishart. O método SEM proposto é flexível para calcular as estimativas das misturas de distribuições Wishart, $\mathcal{K}_{P}$ e $\mathcal{G}_{P}^{0}$.

Analisaram-se dois exemplos: a segmentação da imagem São Francisco com $g=4$ regiões e a segmentação da imagem Munique com $g=3$ regiões. Todos os resultados foram inicializados pelo método quantil. 


\subsubsection{Imagem São Francisco}

Na imagem São Francisco, o método SEM foi executado com 32 iterações e com $95 \%$ do tamanho da imagem. Definiu-se a seleção dos pixels de acordo com da imagem $S P A N$, cujos dados da imagem fossem menores que o quantil amostral $S P A N<Q_{0,95}$. A Tabela 6 fornece os resultados quantitativos obtidos na iteração de maior log-verossimilhança entre as 32 iterações de acordo com as amostras de teste da Figura 2(d) (página 68). Com o método k-médias, utilizou-se o critério de convergência, definido-o pelo erro absoluto entre as médias. A Tabela 7 mostra os resultados quantitativos obtidos na iteração 32 de acordo com as amostras de teste da Figura 2(d). As Figuras 9 e 10 ilustram as segmentações obtidas nas duas iterações analisadas.

Tabela 6- Resultados quantitativos da imagem São Francisco com os métodos SEM, EM e k-médias utilizando a inicialização quantil e $g=4$ regiões: as porcentagem de acertos (P.A.), o coeficiente Kappa $(\widehat{K})$ e a variância do Kappa $\left(\widehat{\sigma^{2}} \widehat{K}\right)$. No método EM e SEM, os resultados foram obtidos na iteração que apresentou a maior $\log$-verossimilhança $\left(\ell_{\max }=\max (\log L(\widehat{\psi}))\right.$ dentre as 32 iterações (Iter.). No método k-médias, os resultados foram obtidos na iteração em que o método convergiu.

\begin{tabular}{ccccccccc}
\hline Método & $\begin{array}{c}\text { P.A.(\%) } \\
\text { Oceano }\end{array}$ & $\begin{array}{c}\text { P.A.(\%) } \\
\text { Floresta }\end{array}$ & $\begin{array}{c}\text { P.A.(\%) } \\
\text { Urbano }\end{array}$ & $\begin{array}{c}\text { P.A.(\%) } \\
\text { Gramado }\end{array}$ & $\begin{array}{c}\text { P.A. }(\%) \\
\text { Total }\end{array}$ & $\widehat{K}$ & $\begin{array}{c}\widehat{\sigma^{2}} \widehat{K} \\
(e-06)\end{array}$ & $\begin{array}{c}\text { Iter. } \\
{ }^{*} \ell_{\max }\end{array}$ \\
\hline SEM $\mathcal{G}_{P}^{0}$ & $\mathbf{9 9 , 7 5}$ & $\mathbf{8 0 , 1 1}$ & 69,74 & 39,54 & $\mathbf{8 4 , \mathbf { 2 6 }}$ & $\mathbf{0 , 7 6}$ & 2,35 & $7^{*}$ \\
SEM $\mathcal{K}_{P}$ & $\mathbf{9 9 , 8 3}$ & 76,77 & $\mathbf{7 1 , 0 7}$ & 39,52 & $\mathbf{8 4 , \mathbf { 1 5 }}$ & $\mathbf{0 , 7 5}$ & 2,36 & $11^{*}$ \\
SEM $W$ & $\mathbf{9 9 , 0 7}$ & 67,21 & 61,02 & 36,80 & 79,61 & 0,68 & 2,74 & $20^{*}$ \\
EM $W$ & $\mathbf{9 9 , 0 7}$ & 67,19 & 60,98 & 36,87 & 79,60 & 0,68 & 2,74 & $20^{*}$ \\
k-médias $W$ & $\mathbf{9 9 , 2 4}$ & 64,98 & 59,75 & $\mathbf{4 5 , 1 8}$ & 79,76 & 0,69 & 2,72 & 10 \\
\hline
\end{tabular}

Tabela 7- Resultados quantitativos da imagem São Francisco com os métodos SEM, EM e k-médias utilizando a inicialização quantil e $g=4$ regiões na iteração 32: as porcentagem de acertos (P.A.), o coeficiente Kappa $(\widehat{K})$ e a variância do Kappa $\left(\widehat{\sigma^{2}} \widehat{K}\right)$.

\begin{tabular}{ccccccccc}
\hline Método & $\begin{array}{c}\text { P.A.(\%) } \\
\text { Oceano }\end{array}$ & $\begin{array}{c}\text { P.A.(\%) } \\
\text { Floresta }\end{array}$ & $\begin{array}{c}\text { P.A.(\%) } \\
\text { Urbano }\end{array}$ & $\begin{array}{c}\text { P.A.(\%) } \\
\text { Gramado }\end{array}$ & $\begin{array}{c}\text { P.A.(\%) } \\
\text { Total }\end{array}$ & $\widehat{K}$ & $\begin{array}{c}\sigma^{2} \widehat{K} \\
(e-06)\end{array}$ & Iter. \\
\hline SEM $\mathcal{G}_{P}^{0}$ & 98,36 & $\mathbf{8 2 , 2 7}$ & $\mathbf{7 8 , 4 5}$ & 14,79 & $\mathbf{8 3 , 9 6}$ & $\mathbf{0 , 7 5}$ & 2,39 & 32 \\
SEM $\mathcal{K}_{P}$ & $\mathbf{9 9 , 7 5}$ & 74,93 & 63,54 & $\mathbf{4 0 , 0 6}$ & 81,99 & 0,72 & 2,49 & 32 \\
SEM $W$ & 98,84 & 65,82 & 63,05 & 34,13 & 79,57 & 0,68 & 2,76 & 32 \\
EM $W$ & 98,83 & 65,72 & 63,07 & 34,09 & 79,56 & 0,68 & 2,76 & 32 \\
k-médias $W$ & 98,22 & 63,50 & 64,11 & 35,91 & 79,33 & 0,68 & 2,78 & 32 \\
\hline
\end{tabular}

De acordo com o Kappa e respectivas variâncias $\left(\widehat{\sigma^{2}} \widehat{K}\right)$, os resultados obtidos com os métodos EM e SEM dos modelos de mistura Wishart são iguais. Estes resultados demonstram que o passo estocástico do método SEM não influencia muito no desempenho do algoritmo quando este é comparado com o método EM. A vantagem do método SEM ocorre por este ser flexível à utilização de distribuições de textura, como a $\mathcal{G}_{P}^{0}$ e $\mathcal{K}_{P}$, que são 


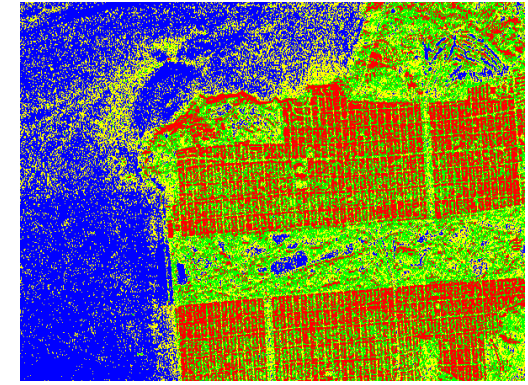

(a) Inicialização Quantil $(g=4)$

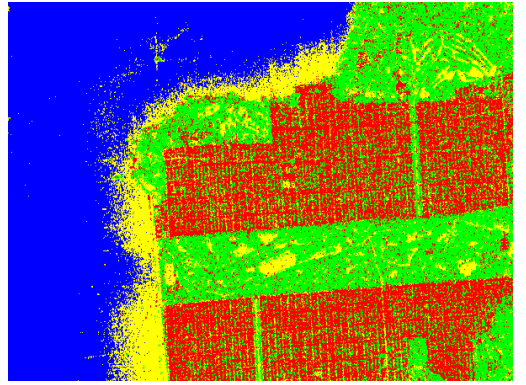

(b) $\operatorname{SEM} \mathcal{G}_{P}^{0}$

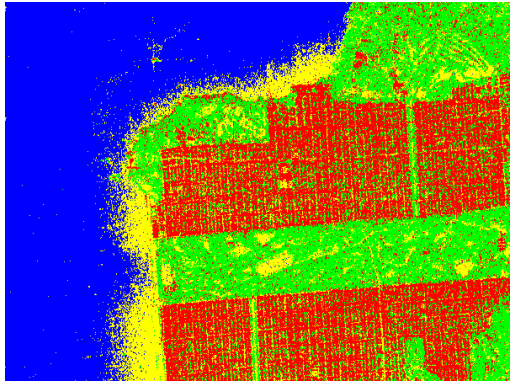

(c) $\operatorname{SEM} \mathcal{K}_{P}$

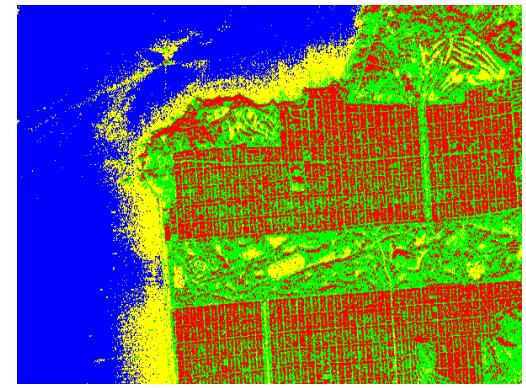

(d) SEM $W$

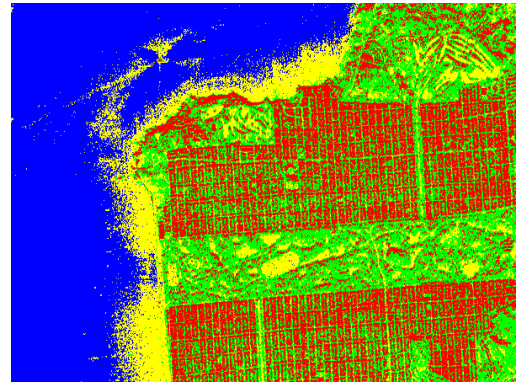

(e) k-médias $W$

Figura 9- Segmentações da imagem São Francisco com a inicialização quantil e $g=4$. Nos métodos SEM, as segmentações foram obtidas na iteração de maior log-verossimilhança. No método k-médias, utilizou-se a iteração de convergência.

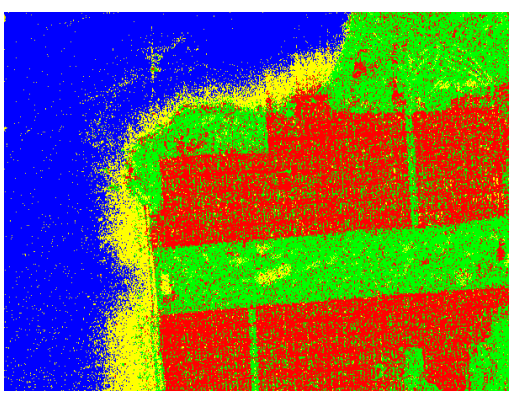

(a) SEM $\mathcal{G}_{P}^{0}$

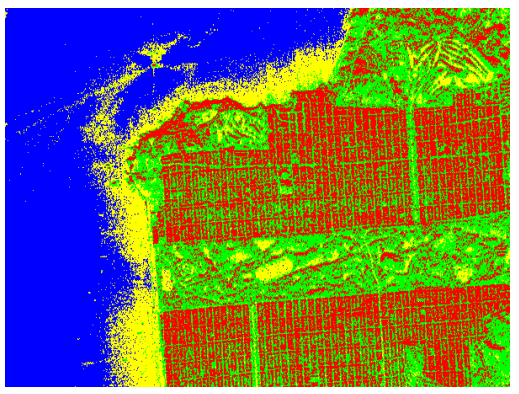

(c) SEM $W$

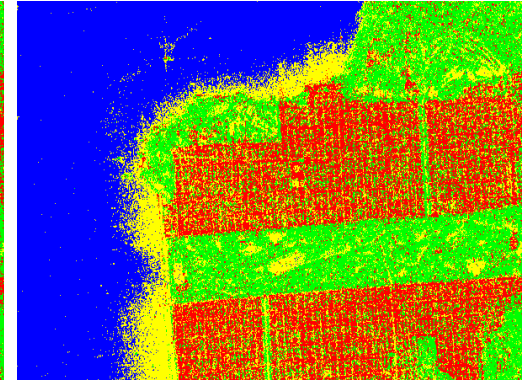

(b) SEM $\mathcal{K}_{P}$

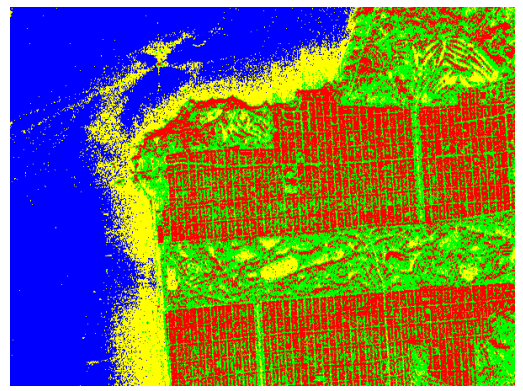

(d) k-médias $W$

Figura 10. Segmentações da imagem São Francisco obtidas na iteração 32 com a inicialização quantil e $g=4$.

distribuições difíceis de serem tratadas analiticamente. As segmentações do método EM não são ilustradas por apresentarem os mesmos resultados do método SEM com a mistura de distribuições Wishart. 
Analisando as segmentações obtidas com a distribuição Wishart, o desempenho do método k-médias diferencia um pouco dos desempenhos dos métodos EM e SEM. O método k-médias utiliza a medida de dissimilaridade contendo apenas termos com o parâmetro $C$. Este fato descarta a influência de outros parâmetros, como o número de looks $\widehat{\eta}$, por exemplo. Enquanto que os métodos EM e SEM utilizam a função densidade de probabilidade da distribuição Wishart. Porém, no geral, estes três métodos apresentam a mesma característica: definem as regiões de acordo com as médias amostrais $\widehat{C}$.

Os melhores resultados da segmentação por SEM, EM e k-médias utilizando a distribuição Wishart são após a convergência destes métodos (coeficientes Kappa 0,68 e 0,69). Há pouca diferença entre os resultados das Tabelas 6 e 7. Há uma melhora na segmentação da região urbana por esta apresentar atributos mais separados que as outras regiões no domínio espacial, como mostram as estimativas das intensidades $\widehat{C}$ na Tabela 2 (página 69). Porém, em todos os resultados com a distribuição Wishart, as regiões de floresta e urbano são misturadas (ver Figuras 9(d), 9(e), 10(c) e 10(d)).

Nas segmentações com a imagem São Francisco, a técnica de agrupamento de dados utilizada não influencia tanto no resultado obtido. O fator influenciador se concentra no tipo de modelo utilizado.

Dentre os modelos utilizados, as distribuições de textura apresentaram os melhores resultados tanto na iteração de maior log-verossimilhança quanto na iteração 32, com destaque na segmentação das regiões de floresta e urbano. Nos modelos de mistura de distribuições $\mathcal{G}_{P}^{0}$, o método de segmentação tende a agrupar a região de floresta com a região de gramado e a destacar a parte litorânea. Além disso, melhora os resultados da região urbana (ver Figuras 9(b) e 10(a)). Nos modelos de mistura de distribuições $\mathcal{K}_{P}$, o método de segmentação mantém o desempenho em algumas regiões, principalmente da região gramado, e piora a segmentação da região urbana (ver Figuras 9(c) e 10(b)).

\subsubsection{Imagem Munique}

Na imagem Munique, o método SEM foi executado com 32 iterações e com $90 \%$ do tamanho da imagem. Definiu-se a seleção dos pixels de acordo com da imagem $S P A N$, cujos dados da imagem pertencessem aos quantis amostrais $Q_{0,05}<S P A N<Q_{0,95}$. A Tabela 8 fornece os resultados quantitativos obtidos na iteração de maior log-verossimilhança entre as 32 iterações de acordo com as amostras de teste da Figura 3(c) (página 69). Com o método k-médias, utilizou-se o critério de convergência, definido-o pelo erro absoluto entre as médias. A Tabela 9 mostra os resultados quantitativos obtidos na iteração 
32 de acordo com as amostras de teste da Figura 3(c). Como a inicialização por quantil forneceu resultado próximo ao da segmentação desejada, calcularam-se as porcentagens de acerto e o Kappa da inicialização para facilitar as análises (ver Tabela 8). As Figuras 25 e 27 (páginas 113 e 115) ilustram as segmentações obtidas nas duas iterações analisadas.

Tabela 8- Resultados quantitativos da imagem Munique com os métodos SEM, EM e k-médias utilizando a inicialização quantil e $g=3$ regiões: porcentagem de acertos (P.A.), coeficiente Kappa $(\widehat{K})$ e variância do Kappa $\left(\widehat{\sigma^{2}} \widehat{K}\right)$. Utilizaram-se $90 \%$ dos pixels da imagem $S P A N$ no intervalo $\left[Q_{0,05}, Q_{0,95}\right]$. As imagens foram segmentadas com a iteração que apresentou a maior log-verossimilhança entre as 32 iterações. No método k-médias foi definida a iteração em que o método convergiu. Há, também, os resultados quantitativos da segmentação inicial por quantis.

\begin{tabular}{cccccccc}
\hline Método & $\begin{array}{c}\text { P.A.(\%) } \\
\text { Cultivo }\end{array}$ & $\begin{array}{c}\text { P.A.(\%) } \\
\text { Vegetação }\end{array}$ & $\begin{array}{c}\text { P.A.(\%) } \\
\text { Urbano }\end{array}$ & $\begin{array}{c}\text { P.A.(\%) } \\
\text { Total }\end{array}$ & $\widehat{K}$ & $\begin{array}{c}\widehat{\sigma^{2}} \widehat{K} \\
(e-05)\end{array}$ & $\begin{array}{c}\text { Iter. } \\
\end{array}$ \\
& $\mathbf{9 8 ,} \ell_{\max }$ \\
\hline SEM $\mathcal{G}_{P}^{0}$ & $\mathbf{6 6 , 5 0}$ & 85,43 & $\mathbf{8 3 , 3 9}$ & $\mathbf{0 , 7 5}$ & 2,16 & $6^{*}$ \\
SEM $\mathcal{K}_{P}$ & 62,75 & 65,81 & 72,39 & 67,08 & 0,51 & 3,49 & $20^{*}$ \\
SEM $W$ & 97,29 & 61,95 & 88,91 & 82,85 & 0,74 & 2,20 & $32^{*}$ \\
EM $W$ & 97,29 & 62,10 & 88,89 & 82,89 & 0,74 & 2,19 & $32^{*}$ \\
k-médias $W$ & 97,81 & 59,19 & $\mathbf{8 9 , 5 7}$ & 82,34 & 0,73 & 2,23 & 10 \\
\hline Inicialização Quantis & 97,76 & 56,79 & 86,81 & 80,60 & 0,71 & 2,37 & - \\
\hline
\end{tabular}

Tabela 9- Resultados quantitativos da imagem Munique com os métodos SEM e k-médias utilizando a inicialização quantil e $g=3$ regiões na iteração 32: porcentagem de acertos (P.A.), coeficiente Kappa $(\widehat{K})$ e variância do Kappa $\left(\widehat{\sigma^{2}} \widehat{K}\right)$. Utilizaram-se $90 \%$ dos pixels da imagem $S P A N$ no intervalo $\left[Q_{0,05}, Q_{0,95}\right]$. Há, também, os resultados quantitativos da segmentação por k-médias com a imagem completa.

\begin{tabular}{cccccccc}
\hline Método & $\begin{array}{c}\text { P.A.(\%) } \\
\text { Cultivo }\end{array}$ & $\begin{array}{c}\text { P.A.(\%) } \\
\text { Vegetação }\end{array}$ & $\begin{array}{c}\text { P.A.(\%) } \\
\text { Urbano }\end{array}$ & $\begin{array}{c}\text { P.A.(\%) } \\
\text { Total }\end{array}$ & $\widehat{K}$ & $\begin{array}{c}\sigma^{2} \widehat{K} \\
(e-05)\end{array}$ & Iter. \\
\hline SEM $\mathcal{G}_{P}^{0}$ & 82,69 & 67,43 & 76,60 & 75,60 & 0,63 & 2,92 & 32 \\
SEM $\mathcal{K}_{P}$ & 66,88 & 63,00 & 71,37 & 67,17 & 0,51 & 3,49 & 32 \\
k-médias $W$ & $\mathbf{9 7 , 6 6}$ & 58,24 & $\mathbf{9 0 , 0 8}$ & $\mathbf{8 2 , 1 6}$ & $\mathbf{0 , 7 3}$ & 2,24 & 32 \\
\hline k-médias $W: 100 \%$ & $\mathbf{9 7 , 7 2}$ & $\mathbf{6 9 , 2 6}$ & 60,46 & 74,97 & 0,63 & 1,99 & 32 \\
\hline
\end{tabular}

Analisando os valores do Kappa e respectivas variâncias dos resultados com a distribuição Wishart, os métodos SEM, EM e k-médias apresentaram resultados semelhantes por segmentarem as regiões baseadas nas médias amostrais $\widehat{C}$. Há melhoras nas segmentações da região de vegetação e, principalmente, da área urbana. Os atributos da região urbana são muito separados dos atributos das regiões cultivo e vegetação, como mostram as estimativas das intensidades $\widehat{C}$ na Tabela 3 (página 70). Na iteração 32 (ver Tabela 9), a segmentação do método k-médias apresenta poucas diferenças quando comparados com o resultado da Tabela 8. Além disso, este método é mais robusto por apresentar uma boa segmentação tanto com a imagem parcial quanto completa (presença dos outliers). Na iteração 32, o método k-médias apresentou as segmentações da imagem parcial com Kappa 0,73 e da imagem completa com Kappa 0,63. Os métodos SEM e EM são 
influenciados pelos valores discrepantes na imagem. As segmentações da imagem completa com todos os métodos de segmentação estão ilustradas na Figura 25 (página 113).

O método SEM com a mistura de distribuições $\mathcal{G}_{P}^{0}$ apresentou a melhor segmentação (Kappa 0,75 ) entre todos os métodos quando a seleção de amostras foi utilizada e na iteração de maior log-verossimilhança. O modelo $\mathcal{G}_{P}^{0}$ descreveu bem a região de vegetação como mostram as Tabelas 8 e 9. Porém com este modelo, a segmentação obtida na iteração 32 misturou as regiões de vegetação e cultivo. O método sofre influências dos parâmetro de rugosidade e da dispersão dos dados na imagem, mesmo com a redução de outliers. A Figura 27 (página 115) ilustra as imagens segmentadas.

O método SEM com a mistura de distribuições $\mathcal{K}_{P}$ forneceu os piores resultados na iteração de maior log-verossimilhança e, também, na iteração 32.

Em Horta et al. (2008), o método SEM do modelo de mistura de distribuições $\mathcal{G}_{P}^{0}$ foi aplicado à segmentação da imagem Munique utilizando as inicializações aleatória e kmédias (distância Euclidiana) e a seleção de amostras, cujas segmentações foram obtidas com $50 \%$ e $25 \%$ do tamanho da imagem.

\subsubsection{Análise do Método SEM com o Dicionário de Famílias}

O método de segmentação SEM também foi analisado com o modelo de misturas do dicionário de famílias $\mathcal{D}=\left\{W, \mathcal{K}_{P}, \mathcal{G}_{P}^{0}\right\}$. As análises foram realizadas com a imagem São Francisco, definindo $g=3$ regiões e considerando todos os pixels da imagem. Utilizaramse três tipos de inicializações: a aleatória, k-médias (distância Euclidiana) e pelos quantis amostrais (ver a Seção 4.3 na página 64). A Figura 7 (página 77) ilustra as partições iniciais das imagens obtidas com os três métodos de inicialização.

Em todos os resultados, o método SEM foi executado com 32 iterações. Porém, os resultados quantitativos foram obtidos na iteração em que o método SEM convergiu. O critério de convergência utilizado foi o erro absoluto relativo entre os passos sucessivos dos estimadores (ver Equação (2.7) da página 23). A Figura 23 (página 110) ilustra as curvas de convergência de alguns parâmetros $\left(\widehat{\boldsymbol{C}}_{h h}, \widehat{\boldsymbol{\alpha}}, \widehat{\boldsymbol{\rho}}\right)$ dos modelos de mistura do dicionário de famílias $\mathcal{D}$.

Os modelos de mistura do dicionário de famílias são adequados aos casos em que o método apresenta muitas flutuações na log-verossimilhança, como acontece com os resultados obtidos com o método SEM do modelo de mistura de distribuições $\mathcal{G}_{P}^{0}$ com a inicialização aleatória. O passo de seleção da família procura pela distribuição que fornece a maior 
log-verossimilhança a cada grupo e, como consequência, aumenta a log-verossimilhança de toda a imagem, como mostra a Figura 11.

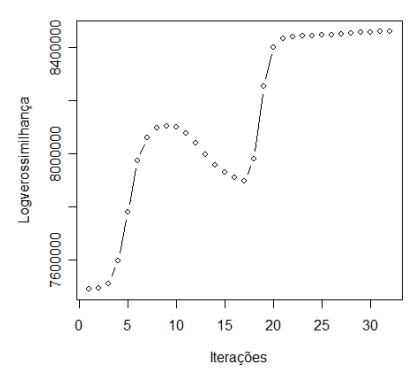

(a) $\mathcal{G}_{P}^{0}$ : Aleatória

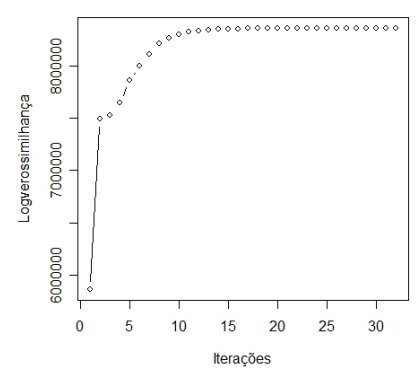

(b) $\mathcal{G}_{P}^{0}\left(\widehat{\alpha}^{(0)}=-50\right)$ : Aleatória

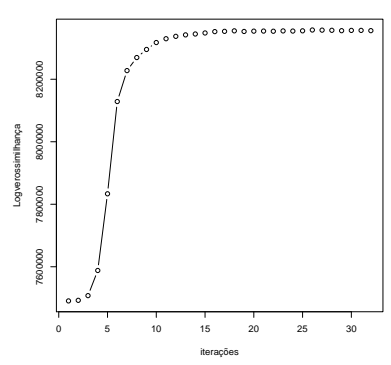

(c) D: Aleatória

Figura 11. Curvas de convergência da log-verossimilhança dos métodos SEM com o modelo de mistura do dicionário de família e com o modelo de mistura de distribuições $\mathcal{G}_{P}^{0}$ aplicado a imagem de São Francisco utilizando $g=3$ regiões e inicialização aleatória.

Neste exemplo, apesar da melhora nos resultados da log-verossimilhança, o método SEM do dicionário de famílias apresentou resultados muito próximos aos do método SEM do modelo de mistura de distribuições $\mathcal{G}_{P}^{0}$. Houve a predominância na seleção da distribuição $\mathcal{G}_{P}^{0}$ para os regiões oceano, vegetação e urbano nos resultados obtidos com os três tipos de inicializações, como mostra a Tabela 11. A distribuição $\mathcal{G}_{P}^{0}$ tem o parâmetro de rugosidade flexível a diferentes tipos de alvo o que pode ter influenciado as escolhas. Os resultados quantitativos estão ilustrados na Tabela 10.

$\mathrm{Na}$ inicialização com os quantis amostrais, a distribuição $\mathcal{K}_{P}$ foi atribuída ao grupo urbano, por isto esta região não foi bem segmentada. Como apresentado nos resultados anteriores (ver Tabela 5 na página 78 ), o parâmetro de rugosidade da distribuição $\mathcal{K}_{P}$ não descreve bem as áreas urbanas quando todos os dados da imagem São Francisco são utilizados. As segmentações estão ilustradas na Figura 12.

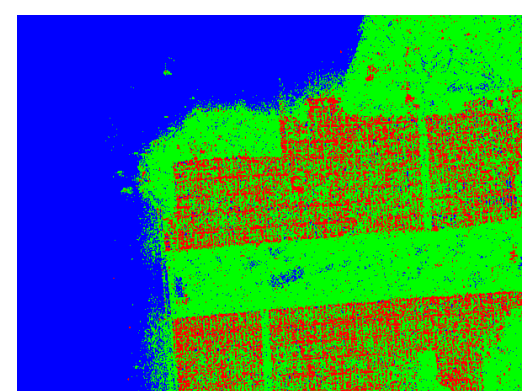

(a) Aleatória: iteração 19

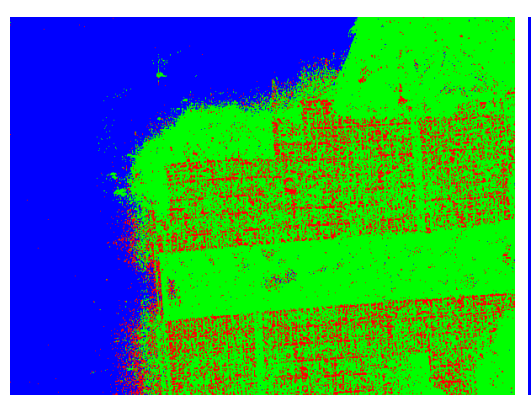

(b) Quantil: iteração 13

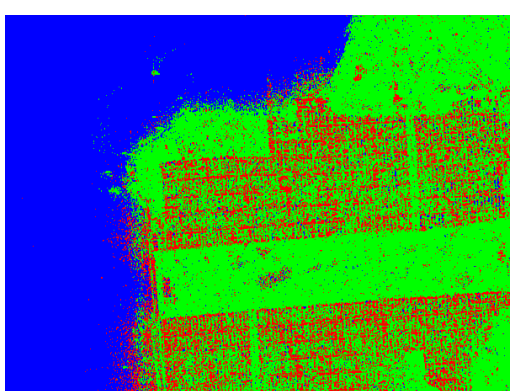

(c) k-médias: iteração 11

Figura 12. Segmentações da imagem São Francisco obtidas na iteração de convergência com método SEM com o dicionário de famílias e as diferentes inicializações com $g=3$. 
Tabela 10- Resultados quantitativos com os métodos SEM do dicionário de famílias e da mistura de distribuições $\mathcal{G}_{P}^{0}$ utilizando as diferentes inicializações da imagem São Francisco contendo $g=3$ regiões. As porcentagem de acertos (P.A.) e coeficiente Kappa foram obtidos na iteração de convergência do método.

\begin{tabular}{|c|c|c|c|c|c|c|c|c|}
\hline $\begin{array}{l}\text { Tipos de } \\
\text { Inicial. }\end{array}$ & $\begin{array}{l}\text { Tipos de } \\
\text { Distrib. }\end{array}$ & $\begin{array}{c}\text { P.A. }(\%) \\
\text { Oceano }\end{array}$ & $\begin{array}{l}\text { P.A.(\%) } \\
\text { Vegetação }\end{array}$ & $\begin{array}{c}\text { P.A.(\%) } \\
\text { Urbano }\end{array}$ & $\begin{array}{c}\text { P.A.(\%) } \\
\text { Total }\end{array}$ & $\widehat{K}$ & $\begin{array}{c}\widehat{\sigma^{2}} \widehat{K} \\
(e-06)\end{array}$ & Iter \\
\hline \multirow{5}{*}{ Aleatória } & $\mathcal{D}$ & $\left(\mathcal{G}_{P}^{0}\right)$ & $\left(\mathcal{G}_{P}^{0}\right)$ & $\left(\mathcal{G}_{P}^{0}\right)$ & & & & \\
\hline & $\mathcal{D}$ & 99,20 & 95,15 & 44,60 & 80,34 & 0,69 & 1,63 & 19 \\
\hline & $\mathcal{G}_{P}^{0}$ & 96,68 & 97,56 & 48,95 & 80,98 & 0,71 & 1,60 & 32 \\
\hline & $\mathcal{G}_{P}^{0 *}$ & 99,20 & 95,22 & 44,55 & 80,34 & 0,69 & 1,63 & 22 \\
\hline & $*\left(\widehat{\boldsymbol{\alpha}}^{(0)}=-50\right)$ & & & & & & & \\
\hline \multirow{3}{*}{ k-médias } & $\mathcal{D}$ & $\left(\mathcal{G}_{P}^{0}\right)$ & $\left(\mathcal{G}_{P}^{0}\right)$ & $\left(\mathcal{G}_{P}^{0}\right)$ & & & & \\
\hline & $\mathcal{D}$ & 99,04 & 92,37 & 45,76 & 80,15 & 0,69 & 1,67 & 11 \\
\hline & $\mathcal{G}_{P}^{0}$ & 99,28 & 94,73 & 46,26 & 80,85 & 0,70 & 1,62 & 11 \\
\hline \multirow{3}{*}{ Quantil } & $\mathcal{D}$ & $\left(\mathcal{G}_{P}^{0}\right)$ & $\left(\mathcal{G}_{P}^{0}\right)$ & $\left(\mathcal{K}_{P}\right)$ & & & & \\
\hline & $\mathcal{D}$ & 95,79 & 96,7 & 34,34 & 75,43 & 0,63 & 1,70 & 13 \\
\hline & $\mathcal{G}_{P}^{0}$ & 96,76 & 97,46 & 51,01 & 81,69 & 0,72 & 1,58 & 08 \\
\hline
\end{tabular}

Tabela 11- Quantidade de seleções das famílias $W, \mathcal{K}_{P}$ e $\mathcal{G}_{P}^{0}$ do método SEM com o modelo do dicionário de famílias e a imagem São Francisco.

\begin{tabular}{ccccc}
\hline Inicialização & Segmento & $W$ & $\mathcal{K}_{P}$ & $\mathcal{G}_{P}^{0}$ \\
\hline \multirow{4}{*}{ Aleatória } & Oceano & 0 & 0 & $\mathbf{3 2}$ \\
& Vegetação & 0 & 0 & $\mathbf{3 2}$ \\
& Urbano & 0 & 2 & $\mathbf{3 0}$ \\
\hline \multirow{4}{*}{ k-médias } & Oceano & 0 & 2 & $\mathbf{3 0}$ \\
& Vegetação & 0 & 0 & $\mathbf{3 2}$ \\
& Urbano & 0 & 2 & $\mathbf{3 0}$ \\
\hline \multirow{4}{*}{ Quantil } & Oceano & 0 & 0 & $\mathbf{3 2}$ \\
& Vegetação & 0 & 0 & $\mathbf{3 2}$ \\
& Urbano & 0 & $\mathbf{3 2}$ & $\mathbf{0}$ \\
\hline
\end{tabular}




\section{Capítulo 6}

\section{Conclusão}

Esta tese apresentou um método de segmentação de imagens SAR polarimétricas multilook utilizando os modelos de mistura de distribuições Wishart, $\mathcal{K}_{P}$ e $\mathcal{G}_{P}^{0}$. Para isto, combinou-se o método de agrupamento de dados SEM com o método dos momentos para estimar os parâmetros dos modelos de mistura. O método SEM também foi adaptado à mistura de um dicionário de famílias contendo essas três distribuições.

Analisou-se a viabilidade do método SEM aos quatro modelos de mistura de distribuições de acordo com a convergência da log-verossimilhança e dos parâmetros dos modelos. As análises foram realizadas com duas imagens PolSAR multi-look reais obtidas na banda $L$ e três métodos de inicializações, que são: aleatório, k-médias (distância Euclidiana) e quantil.

Com o método SEM, há comparações entre os modelos de mistura de distribuições de Wishart, $\mathcal{K}_{P}, \mathcal{G}_{P}^{0}$ e do dicionário de famílias. Além disso, o método SEM foi comparado com dois métodos propostos na literatura, os métodos EM e k-médias que utilizam a distribuição Wishart.

Os resultados de convergência do método SEM mostram que a log-verossimilhança estabiliza com valores próximos do valor máximo quando algumas condições são satisfeitas:

- Inicialização: utilizar o método de quantis amostrais ou por k-médias (distância Euclidiana) que apresentam regiões mais separadas no domínio espacial. Se a inicialização aleatória for utilizada com os modelos de mistura de distribuições $\mathcal{K}_{P}$ e $\mathcal{G}_{P}^{0}$, deve-se definir as estimativas iniciais do parâmetro de rugosidade com valores altos $|\widehat{\boldsymbol{\alpha}}| \rightarrow \infty$. Os parâmetros de rugosidade são influenciados pela inicialização aleatória e, por conseguinte, a convergência do método é afetada.

- Seleção de amostras: eliminar outliers (valores discrepantes na imagem). Quando os atributos da imagem são muito dispersos espacialmente, o método SEM tende a 
definir grupos de outliers.

Se essas condições não forem satisfeitas, a curva de convergência da log-verossimilhança do método SEM pode fornecer flutuações abruptas e que não condizem com as propriedades deste algoritmo. Porém, mesmo com estes problemas na curva de convergência da log-verossimilhança, verificou-se que a segmentação na iteração de maior log-verossimilhança fornece os melhores resultados entre as segmentações obtidas em todo o método. Então, é mais efetivo segmentar a imagem na iteração de máxima log-verossimilhança em todas as situações para garantir que o método forneça o melhor resultado.

Ao comparar os métodos de inicializações aleatório, k-médias e quantil, o método SEM com os diferentes modelos de mistura apresentou os melhores resultados utilizando a inicialização por quantis amostrais. A inicialização por quantis é um método simples e com baixo custo computacional. A aplicação do SEM com os diferentes métodos de inicialização apresentou resultados semelhantes em cada modelo de mistura. A diferença do desempenho do método foi no número de iterações necessárias até a sua convergência. A inicialização aleatória necessita de muitas iterações por causa da sua natureza estocástica.

Dentre as análises entre os modelos de mistura de distribuições Wishart, $\mathcal{K}_{P}$ e $\mathcal{G}_{P}^{0}$, o método SEM com a mistura de distribuições $\mathcal{G}_{P}^{0}$ forneceu os melhores resultados. Esta distribuição apresenta um parâmetro de rugosidade que é flexível aos diferentes graus de homogeneidade das distribuições. A distribuição Wishart é robusta à modelagem dos dados com as diferentes inicializações, mas não é tão flexível à variabilidade de alvos mais heterogêneos, como florestas. O parâmetro de rugosidade das distribuições $\mathcal{K}_{P}$ foi o mais influenciado às diferentes análises. Em alguns casos, o método SEM com o modelo $\mathcal{K}_{P}$ apresentou as piores segmentações.

Nas comparações entre os diferentes métodos SEM, EM e k-médias (distância Wishart), verificou-se que o método SEM com a mistura de distribuições $\mathcal{G}_{P}^{0}$ forneceu os melhores resultados na iteração de máxima log-verossimilhança e com a eliminação dos outliers, destacando-se na segmentação de regiões heterogêneas, como as áreas de vegetação e floresta.

O método EM com mistura de distribuições Wishart teve o desempenho parecido com o método SEM com mistura de Wishart, mostrando que o passo adicional estocástico do método SEM não influência na segmentação. O passo estocástico controla as flutuações presentes na log-verossimilhança já que alguns parâmetros foram definidos pelo método dos momentos. O método k-médias se mostrou robusto por apresentar boas segmentações tanto com a presença de outliers quanto na ausência destes.

Nas análises com os modelos de mistura do dicionário de famílias Wishart, $\mathcal{K}_{P}$ e $\mathcal{G}_{P}^{0}$, verificou-se que a distribuição $\mathcal{G}_{P}^{0}$ é flexível para modelar os diferentes tipos de regiões. 
Nos resultados com as diferentes inicializações e regiões, houve a predominância na seleção da distribuição $\mathcal{G}_{P}^{0}$ no dicionário de famílias. O modelo do dicionário de famílias pode ser uma alternativa à aplicação do método SEM com a inicialização aleatória, pois melhora a curva de convergência da log-verossomilhança.

Diante de todas as análises realizadas, os resultados mostram que o método SEM proposto é flexível aos modelos de mistura de distribuições Wishart, $\mathcal{K}_{P}, \mathcal{G}_{P}^{0}$ e do dicionário de famílias contendo estas três distribuições. Além disso, sua aplicação à segmentação de imagens PolSAR multi-look forneceu resultados característicos a cada tipo distribuição utilizada.

\subsection{Trabalhos Futuros}

O método SEM com os estimadores do método dos momentos se mostrou flexível aos modelos de mistura Wishart, $\mathcal{K}_{P}, \mathcal{G}_{P}^{0}$, mas a log-verossimilhança do método é infuênciada pela inicialização e pela presença de valores discrepantes na imagem. Desta forma, apresentam-se como trabalhos futuros:

- Analisar a aplicação do método SEM à segmentação de imagens PolSAR multi-look reais adquiridas em diferentes bandas. Nesta tese, as duas imagens reais estudadas foram adquiridas na banda $L$;

- Definir critérios de seleção de amostras para tornar o método SEM mais robusto aos valores discrepantes de imagens PolSAR multi-look;

- Definir critérios de seleção do número de distribuições $(g)$ para tornar a segmentação completamente não supervisionada;

- Analisar o desempenho do método com a inicialização por decomposição de CloudePottier, diante do crescente avanço de pesquisas na área (CAO et al., 2007; LEE; POTTIER, 2009);

- Analisar o desempenho do método SEM com a recente distribuição de textura KummerU (BOMBRUN; BEAULIEU, 2008);

- Analisar a possibilidade de incluir uma etapa contextual ao método. 


\section{Referências}

ALFÒ, M.; NIEDDU, L.; VICARI, D. A finite mixture model for image segmentation. Statistics and Computing, v. 18, n. 2, p. 137-150, 2008.

AYED, I. B.; MITICHE, A.; BELHADJ, Z. Polarimetric image segmentation via maximum-likelihood approximation and efficient multiphase level-sets. IEEE Transactions on Pattern Analysis and Machine Intelligence, v. 28, n. 9, p. 1493-1500, 2006.

BEAULIEU, J. M.; TOUZI, R. Segmentation of textured polarimetric SAR scenes by likelihood approximation. IEEE Transactions on Geoscience and Remote Sensing, v. 42, n. 10, p. 2063-2072, 2004.

BIERNACKI, C.; CELEUX, G.; GOVAERT, G. Choosing starting values for the EM algorithm for getting the highest likelihood in multivariate Gaussian mixture models. Computational Statistics \& Data Analysis, v. 41, n. 3, p. 561-575, 2003.

BOMBRUN, L.; BEAULIEU, J.-M. Fisher distribution for texture modeling of polarimetric SAR data. IEEE Geoscience and Remote Sensing Letters, v. 5, n. 3, p. 512-516, 2008.

BOUGUILA, N.; ZIOU, D. A hybrid SEM algorithm for high-dimensional unsupervised learning using a finite generalized Dirichlet mixture. IEEE Transactions on Image Processing, v. 15, n. 9, p. 2657-2668, 2006.

BUTLER, R. W. Predictive likelihood inference with applications. Journal of the Royal Statistical Society B, v. 48, n. 1, p. 1-38, 1986.

$\mathrm{CAO}$, F. et al. An unsupervised segmentation with an adaptive number of clustersusing the SPAN $/ \mathrm{H} / \alpha / \mathrm{A}$ space and the Complex Wishart clusteringfor fully polarimetric SAR data analysis. IEEE Transactions on Geoscience and Remote Sensing, v. 45, n. 11, p. 3454-3467, 2007.

CELEUX, G.; DIEBOLT, J. The SEM algorithm: a probabilistic teacher algorithm derived from the EM algorithm for the mixture problem. Computational Statistics Quaterly, v. 2, n. 1, p. 73-82, 1985. 
CONGALTON, R. G.; GREEN, K. Assessing the accuracy of remotely sensed data: principles and practices. 2. ed. Boca Raton: CRC Press, 2009. 183 p.

CORREIA, A. H. Projeto, desenvolvimento e avaliação de classificadores estatísticos pontuais e contextuais para imagens SAR polarimétricas. 1998. 271 p. Dissertação (Mestrado em Sensoriamento Remoto) - Instituto Nacional de Pesquisas Espaciais, São José dos Campos, 1998.

COSTA, L. F.; JUNIOR, R. M. C. Shape analysis and classification: theory and practice. Boca Raton: CRC Press, 2001. 659 p.

DAY, N. E. Estimating the components of a mixture of two normal distributions. Biometrika, v. 56, n. 3, p. 463-474, 1969.

DEGROOT, M. H.; SCHERVISH, M. J. Probability and statistics. 3. ed. New York: Addison Wesley, 2002. 816 p.

DELIGNON, Y.; MARZOUKI, A.; PIECZYNSKI, W. Estimation of generalized mixture and its application in image segmentation. IEEE Transactions on Image Processing, v. 6, n. 10, p. 1364-1375, 1997.

DEMPSTER, A. P.; LAIRD, N. M.; RUBIN, D. B. Maximum likelihood from incomplete data via the EM algorithm. Journal of the Royal Statistical Society B, v. 39, n. 1, p. 1-38, 1977.

DERRODE, S.; PIECZYNSKI, W. Signal and image segmentation using pairwise Markov chains. IEEE Transactions on Signal Processing, v. 59, n. 9, p. 2477-2489, 2004.

DONG, Y.; MILNE, A. K.; FORSTER, B. C. Segmentation and classification of vegetated areas using polarimetric SAR image data. IEEE Transactions on Geoscience and Remote Sensing, v. 39, n. 2, p. 321-329, 2001.

DUDA, R. O.; HART, P. E.; STORK, D. G. Pattern classification. 2. ed. New York: John Wiley \& Sons, 2001. 654 p.

EVERITT, B. S.; HAND, D. J. Finite mixture distributions. New York: Chapman \& Hall, 1981. 143 p.

FIGUEIREDO, M. A. T.; JAIN, A. K. Unsupervised learning of finite mixture models. IEEE Transactions on Pattern Analysis and Machine Intelligence, v. 24, n. 3, p. 381-396, 2002. 
FREITAS, C. C.; FRERY, A. C.; CORREIA, A. H. The polarimetric $\mathcal{G}$ distribution for SAR data analysis. Environmetrics, v. 16, n. 1, p. 13-31, 2005.

FRERY, A. C.; CORREIA, A. H.; FREITAS, C. C. Classifying multifrequency fully polarimetric imagery with multiplesourcesof statistical evidence and contextual information. IEEE Transactions on Geoscience and Remote Sensing, v. 45, n. 10, p. 3098-3109, 2007.

FRERY, A. C. et al. A model for extremely heterogeneous clutter. IEEE Transactions on Geoscience and Remote Sensing, v. 35, n. 3, p. 648-659, 1997.

GAMBINI, J. et al. Feature extraction in speckled imagery using dynamic B-spline deformable contours under the G0 model. International Journal of Remote Sensing, v. 27, n. 22, p. 5037-5059, 2006.

GAMBINI, J. et al. Accuracy of edge detection methods with local information in speckled imagery. Statistics and Computing, v. 18, n. 1, p. 15-26, 2008.

GONZALEZ, R. C.; WOODS, R. E. Digital image processing. 2. ed. New Jersey: Prentice-Hall, 2002. 793 p.

HASSELBLAD, V. Estimation of parameters for a mixture of normal distributions. Technometrics, v. 8, n. 3, p. 431-444, 1966.

HASSELBLAD, V. Estimation of finite mixtures of distributions from the Exponencial family. Journal of American Statistical Association, v. 65, n. 328, p. 1459-1471, 1969.

HORTA, M. M.; MASCARENHAS, N. D. A.; FRERY, A. C. Analysing polarimetric imagery with G0p mixture models and SEM algorithm. In: BRAZILIAN SYMPOSIUM ON COMPUter graphics ANd image PRocessing, Sibgrapi, 20., 2007, Belo Horizonte. Anais... Belo Horizonte: [s.n.], 2007. p. 71-72 (Posters).

HORTA, M. M.; MASCARENHAS, N. D. A.; FRERY, A. C. Classification of polarimetric SAR imagery with mixtures of distributions. In: URSI COMMISSION F TRIENNIAL OPEN SYMPOSIUM ON RADIO WAVE PROPAGATION AND REMOTE SENSING, URsi, 11., 2007, Rio de Janeiro. Proceedings... Rio de Janeiro: [s.n.], 2007. p. 144-149.

HORTA, M. M.; MASCARENHAS, N. D. A.; FRERY, A. C. A comparison of clustering fully polarimetric SAR images using SEM algorithm and G0p mixture model with different initializations. In: INTERNATIONAL CONFERENCE ON PATTERN ReCognition,ICPR, 19., 2008, Tampa. Proceedings... Tampa: IEEE, 2008. p. 1-4. 
HORTA, M. M. et al. Clustering of fully polarimetric SAR data using finite G0p mixturemodel and SEM algorithm. In: INTERNATIONAL CONFERENCE ON SYSTEMS Signals And image PRocessing, IWssip, 15., 2008, Bratislava. Proceedings... Bratislava: IEEE, 2008. p. 81-84.

HYNDMAN, R. J.; FAN, Y. Sample quantiles in statistical packages. The American Statistician, v. 50, n. 4, p. 361-365, 1996.

JACOBO-BERLLES, J. Nuevas distribuciones para datos polarimétricos de radar de apertura sintética. 2005. 79 p. Tese (Doctorado en Ciencias de la Computación) Departamento de Computación, Universidad de Buenos Aires, Buenos Aires, 2005.

JAKEMAN, E.; PUSEY, P. N. A model for non-Rayleigh sea echo. IEEE Transactions on Antennas and Propagation, v. 24, n. 6, p. 806-814, 1976.

JEFFREYS, H. An alternative to the rejection of observations. Proceedings of the Royal Society of London A, v. 137, n. 831, p. 78-87, 1932.

KERSTEN, P. R.; LEE, J. S.; AINSWORTH, T. L. Unsupervised classification of polarimetric synthetic aperture radarimages using fuzzy clustering and EM clustering. IEEE Transactions on Geoscience and Remote Sensing, v. 43, n. 3, p. 519-527, 2005.

KHAN, K. U.; YANG, J.; ZHANG, W. Unsupervised classification of polarimetric SAR images by EMalgorithm. IEICE Transactions on Communications, E90-B, n. 12, p. 3632-3642, 2007.

LEE, J. S.; GRUNES, M. R.; KWOK, R. Classification of multi-look polarimetric SAR imagery based on complex Wishart distributions. International Journal of Remote Sensing, v. 15, n. 11, p. 2299-2311, 1994.

LEE, J. S. et al. Intensity and phase statistics of multilook polarimetric and interferometric SAR imagery. IEEE Transactions on Geoscience and Remote Sensing, v. 32, n. 5, p. 1017-1028, 1994a.

LEE, J. S.; POTTIER, E. Polarimetric radar imaging: from basics to applications. Boca Raton: CRC Press, 2009. 398 p.

LEE, J. S. et al. Scattering-model-based speckle filtering of polarimetric SAR data. IEEE Transactions on Geoscience and Remote Sensing, v. 44, n. 1, p. 176-187, 2006.

LEE, J. S. et al. K distribution for multi-look processed polarimetric SAR imagery. In: INTERNATIONAL GEOSCIENCE AND REMOTE SENSING SYMPOSIUM, IGARSS, 1994, Pasadena. Proceedings... Pasadena: IEEE, 1994b. v. 4, p. 2179-2181. 
LEE, J. S. et al. Improved sigma filter for speckle filtering of SAR imagery. IEEE Transactions on Geoscience and Remote Sensing, v. 47, n. 1, p. 202-213, 2009.

MCLACHLAN, G. J.; KRISHNAN, T. The EM algorithm and extensions. New York: John Wiley \& Sons, 1997. 274 p.

MCLACHLAN, G. J.; PEEL, D. Finite mixture models. New York: John Wiley \& Sons, 2000. 419 p.

MIGNOTTE, M. et al. Sonar image segmentation using an unsupervised hierarchical MRF model. IEEE Transactions on Image Processing, v. 9, n. 7, p. 1216-1231, 2000.

MOSER, G.; ZERUBIA, J.; SERPICO, S. B. Dictionary-based stochastic expectationmaximization for SAR amplitude probability density function estimation. IEEE Transactions on Geoscience and Remote Sensing, v. 44, n. 1, p. 188-200, 2006.

MULLER, H. J. et al. The harmonic branch of the multiplicative model: properties and applications. In: EUROPEAN CONFERENCE ON SYNTHETIC APERTURE RADAR, EUSAR, 3., 2000, München. Proceedings... München: VDE, 2000. p. 603-606.

NASCIMENTO, A. D. C. Medidas de contraste e distâncias estocásticas em modelos para dados com ruído speckle, 2008. 149p. Dissertação (Mestrado em Estatística) Universidade Federal de Pernambuco, Recife, 2008.

NASCIMENTO, A. D. C.; CINTRA, R. J. S.; FRERY, A. C. Stochastic distances and hypothesis testing in speckled data. In: BRAZILIAN REMOTE SENSING SYMPOSIUM, SBsR, XIV., 2008, Natal. Proceedings... Natal: in press, 2009.

NEWCOMB, S. A generalized theory of the combination of observations as to obtain the best result. American Journal of Mathematics, v. 8, n. 4, p. 343-366, 1886.

OLIVER, C.; QUEGAN, S. Understanding synthetic aperture radar images. Boston: Artech House, 1998. 479 p.

ORCHARD, T.; WOODBURY, M. A. A missing information principle: theory and applications. In: BERKELEY SYMPOSIUM ON MATHEMATICAL, 6., 1972, Berkeley. Proceedings... Berkeley: University of California Press, 1972. v. 1, p. 697-715.

PRATT, W. K. Digital image processing. 3. ed. New York: John Wiley \& Sons, 2001. $656 \mathrm{p}$.

REDNER, R. A.; WALKER, H. F. Mixture densities, maximum likelihood and the EM algorithm. SIAM Review, v. 26, n. 2, p. 195-239, 1984. 
RENNÓ, C. D. Avaliação de medidas texturais na discriminação de classes de usoutilizando imagens SIR-C/X-SAR do perimetro irrigado de Bebedouro, Petrolina, PE. 1995. 107 p. Dissertação (Mestrado em Sensoriamento Remoto) — Instituto Nacional de Pesquisas Espaciais, São José dos Campos, 1995.

SKRIVER, H. et al. Polarimetric segmentation using Wishart test statistic. In: INTERNATIONAL GEOSCIENCE AND REMOTE SENSING SYMPOSIUM, IGARSS, 2002, Toronto. Proceedings... Toronto: IEEE, 2002. v. 2, p. 1011-1013.

TISON, C. et al. A new statistical model for Markovian classification of urban areas in high-resolution SAR images. IEEE Trasnsactions on Geoscience and Remote Sensing, v. 42, n. 10, p. 2046-2057, 2004.

TITTERINGTON, D. M.; SMITH, A. F. M.; MAKOV, U. E. Statistical analysis of finite mixture distributions. New York: John Wiley \& Sons, 1985. 254 p.

TOUZI, R. et al. A review of polarimetry in the context of synthetic aperture radar:concepts and information extraction. Canadian Journal of Remote Sensing, v. 30, n. 3, p. 380-407, 2004.

TSO, B.; MATHER, P. M. Classification methods for remotely sensed data. London: Taylor \& Francis, 2001. 356 p.

ULABLY, F. T.; ELACHI, C. Radar polarimetry for geoscience applications. Norwood: Artech House, 1990. 376 p.

VASILE, G. et al. Intensity-driven-adaptive-neighborhood technique for polarimetric and interferometric parameter estimation. IEEE Transactions on Geoscience and Remote Sensing, v. 44, n. 4, p. 994-1003, 2006.

WOLFE, J. H. A computer program for the maximum likehood analysis of types. San Diego: U. S. Naval Personnel Research Activity, 1965. p. 1-52 (Technical Bulletin, 65-15).

WOLFE, J. H. Normix: computational methods for estimating the parameters of multivariate normal mixtures of distributions. San Diego: U. S. Naval Personnel Research Activity, 1967. p. 1-38 (Research Memorandum SRM 68-2).

WOLFE, J. H. Pattern clustering by multivariate mixture analysis. Multivariate Behavioral Research, v. 5, n. 3, p. 329-350, 1970.

WU, C. F. J. On the convergence properties of the EM algorithm. Annals of Statistics, v. 11, n. 1, p. 95-103, 1983. 
YANG, X.; KRISHNAN, S. M. Image segmentation using finite mixtures and spatial information. Image and Vision Computing, v. 22, n. 9, p. 735-745, 2004.

YANG, X.; LIU, J. Mixture density estimation with group membership functions. Pattern Recognition Letters, v. 23, n. 5, p. 501-512, 2002.

YU, Y.; ACTON, S. T. Polarimetric SAR image segmentation using texture partitioning and statstical analysis. In: INTERNATIONAL CONFERENCE ON IMAGE PROCESSING, ICIP, 2000, Vancouver. Proceedings... Vacouver: IEEE, 2000. v. 1, p. 677-680. 


\section{APÊNDICE A - Análise Descritiva dos Atributos das Imagens}

Este apêndice fornece algumas estatísticas sobre os atributos das imagens de São Francisco e Munique.

\section{A.1 Imagem de São Francisco}

As Figuras 13 e 14 ilustram o gráfico de dispersão dos canais de intensidade da imagem São Francisco e o histograma da imagem $S P A N$. No caso da imagem $S P A N$, a análise descritiva dos dados é: valor mínimo $(6,2 e-04)$, primeiro quartil $\left(Q_{0,25}=2,7 e-02\right)$, mediana $(8,9 e-02)$, média $(2,5 e-01)$, terceiro quartil $\left(Q_{0,75}=2,4 e-01\right)$ e valor máximo $(6,1 e+01)$.

\section{A.2 Imagem Munique}

As Figuras 15 e 16 ilustram o gráfico de dispersão dos canais de intensidade da imagem Munique o histograma da imagem $S P A N$. No caso da imagem $S P A N$, a análise descritiva dos dados é: valor mínimo $(1,2 e+04)$, primeiro quartil $\left(Q_{0,25}=4,3 e+05\right)$, mediana $(1,1 e+06)$, média $(6,7 e+06)$, terceiro quartil $\left(Q_{0,75}=2,7 e+06\right)$ e valor máximo $(8,8 e+09)$. 


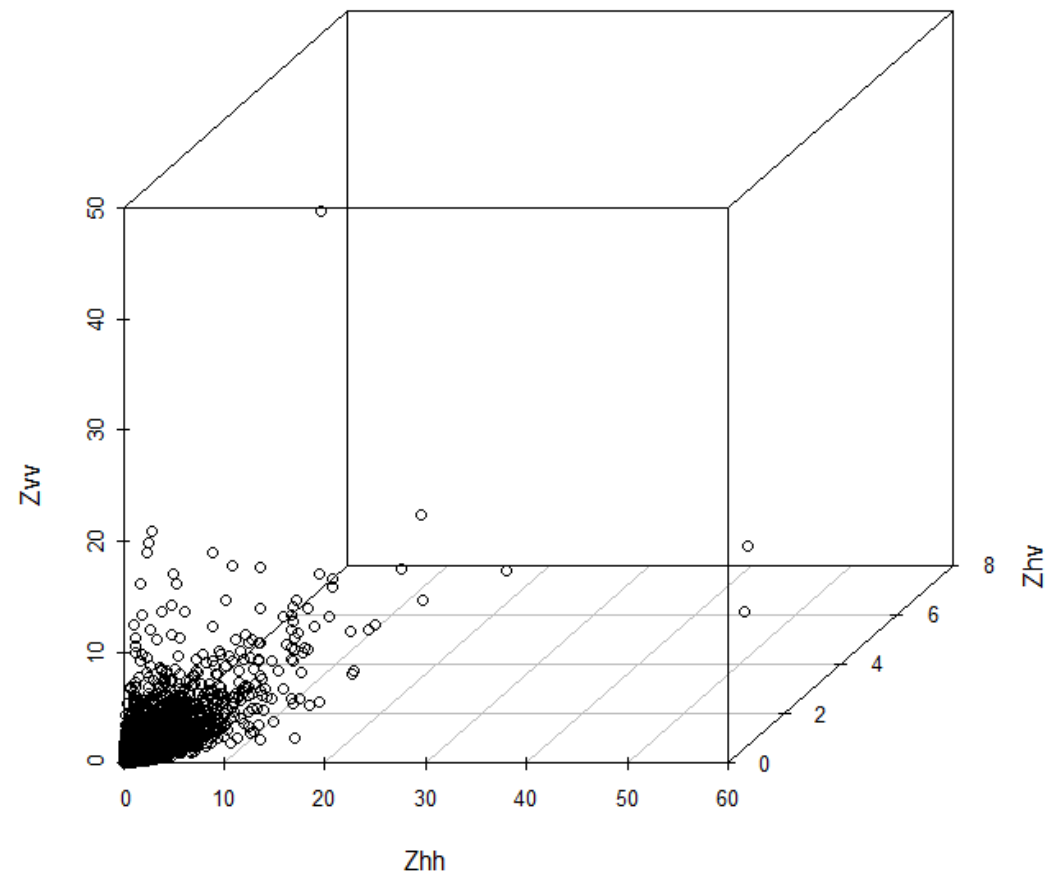

Figura 13. Gráfico de dispersão dos canais de intensidade da imagem de São Francisco.

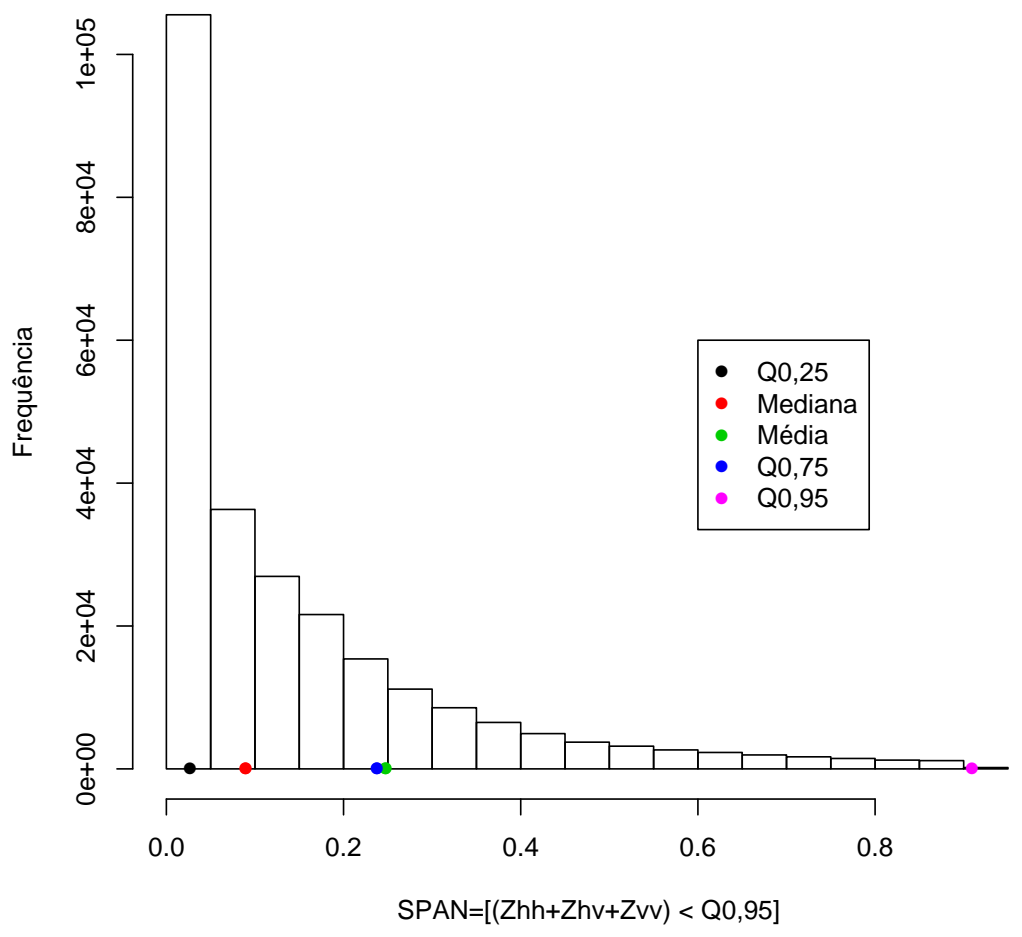

Figura 14. Histograma da imagem $S P A N$ de São Francisco. 


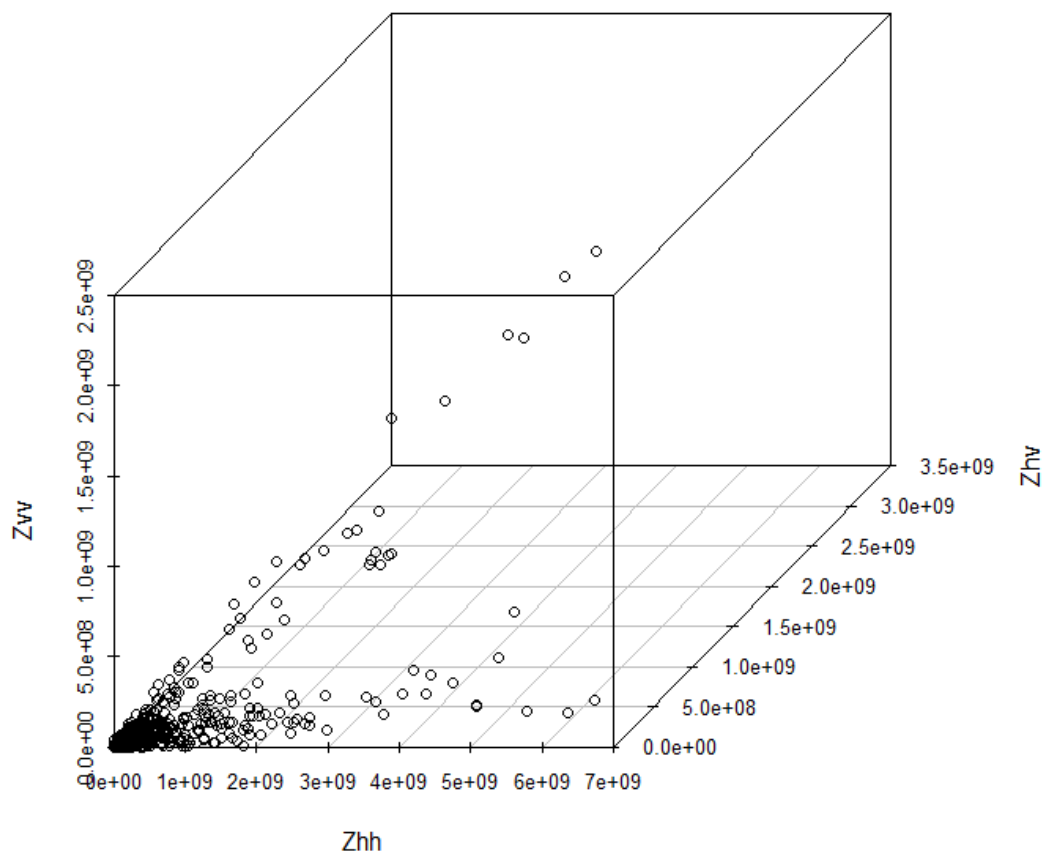

Figura 15. Gráfico de dispersão dos canais de intensidade da imagem de Munique.

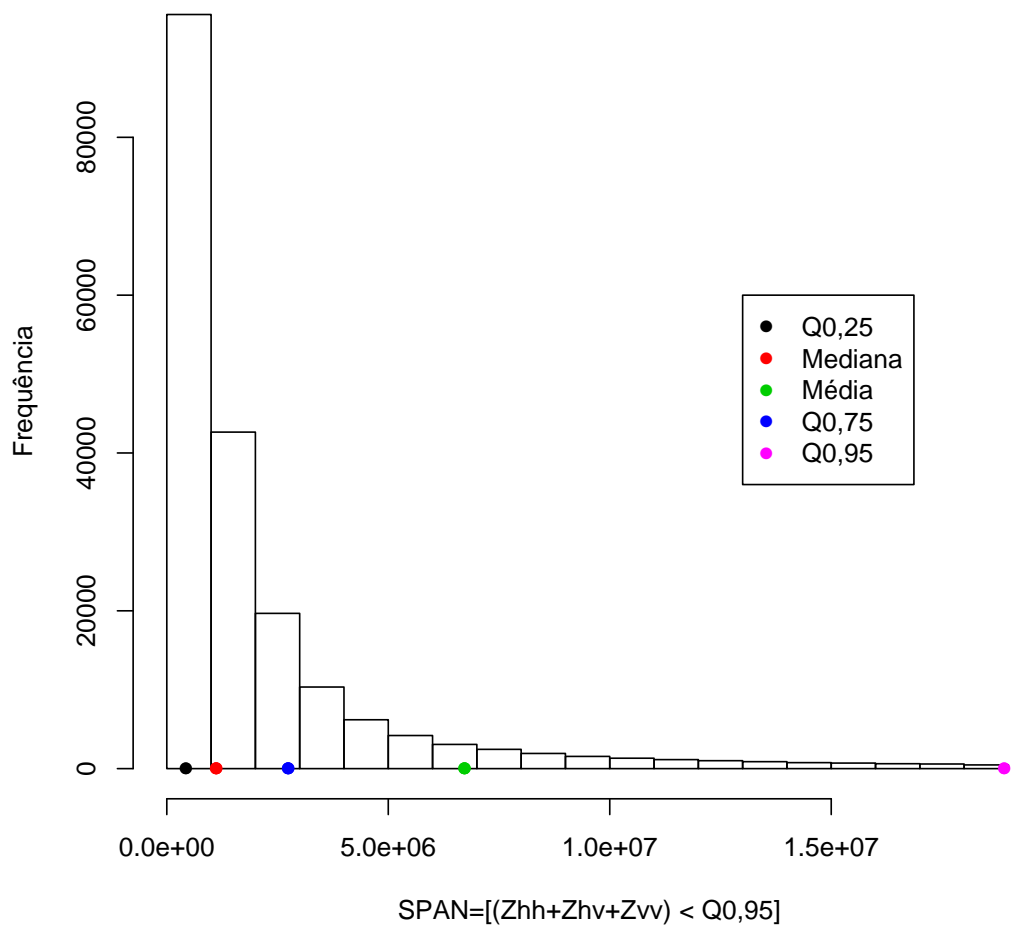

Figura 16. Histograma da imagem $S P A N$ de Munique. 


\section{APÊNDICE B - Resultados da Imagem São Francisco}

Este apêndice apresenta os resultados obtidos com o método SEM dos modelos de mistura $\mathcal{G}_{P}^{0}, \mathcal{K}_{P}$ e Wishart e do dicionário de famílias $\mathcal{D}$ com as inicializações aleatória, k-médias (distância Euclidiana) e quantis amostrais.

As Figuras 17, 19 e 21 ilustram as curvas de convergência da log-verossimilhança $(\ell(\widehat{\psi}))$ e dos parâmetros dos modelos de mistura $(\widehat{\psi})$ das distribuições Wishart, $\mathcal{K}_{P}$ e $\mathcal{G}_{P}^{0}$.

As Figuras 18, 20 e 22 ilustram as imagens segmentadas com os modelos de mistura das distribuições Wishart, $\mathcal{K}_{P}$ e $\mathcal{G}_{P}^{0}$.

A Figura 23 ilustra as curvas de convergência da log-verossimilhança $(\ell(\widehat{\psi}))$ e dos parâmetros dos modelos de mistura $(\widehat{\psi})$ do dicionário de famílias $\mathcal{D}=\left\{W, \mathcal{K}_{P}, \mathcal{G}_{P}^{0}\right\}$. 


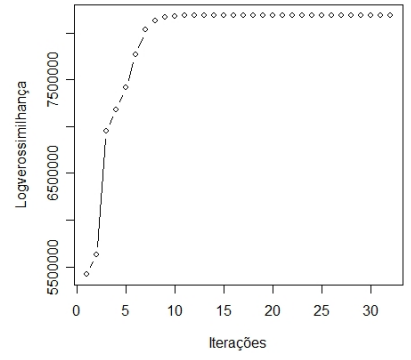

(a) $W: \ell(\widehat{\psi})$

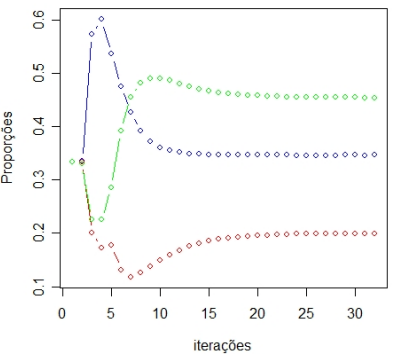

(b) $W: \widehat{\rho}$

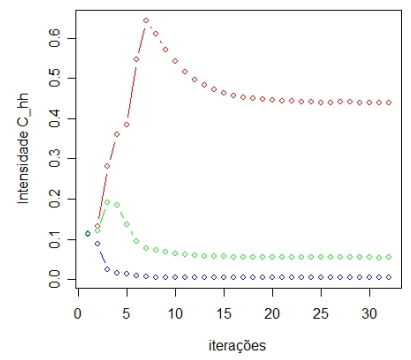

(c) $W: \widehat{C}_{h h}$

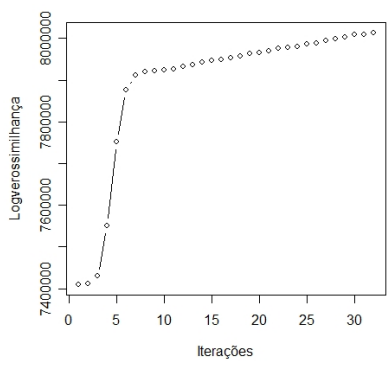

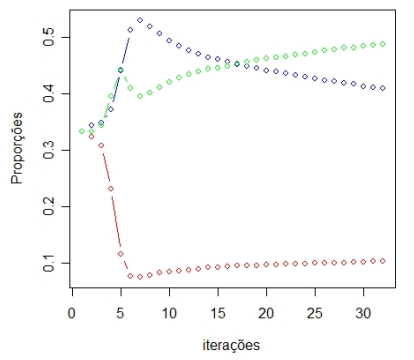

(e) $\mathcal{K}_{P}: \widehat{\rho}$

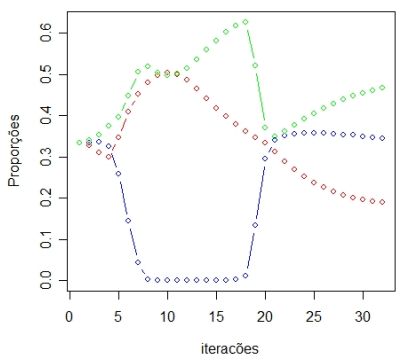

(i) $\mathcal{G}_{P}^{0}: \widehat{\rho}$

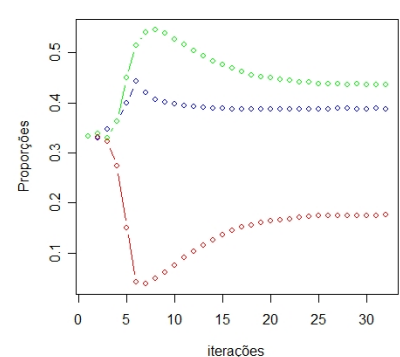

$(\mathbf{m}) \mathcal{G}_{P}^{0}\left(\widehat{\alpha}^{(0)}=-50\right): \hat{\rho}$

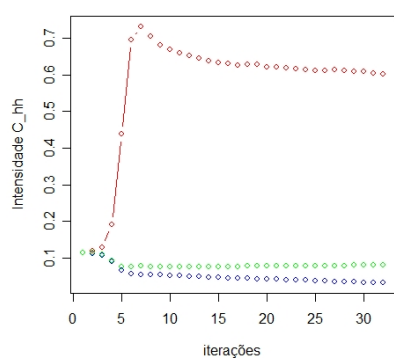

(f) $\mathcal{K}_{P}: \widehat{C}_{h h}$

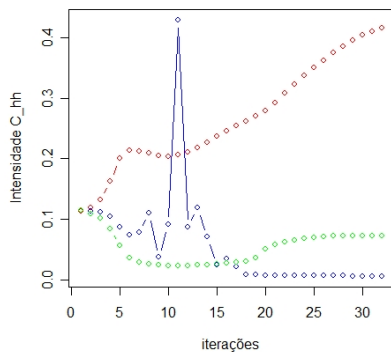

(j) $\mathcal{G}_{P}^{0}: \widehat{\boldsymbol{C}}_{h h}$

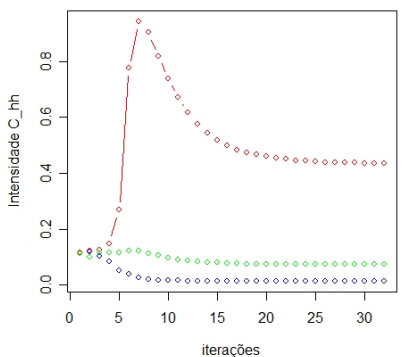

(n) $\mathcal{G}_{P}^{0}\left(\widehat{\alpha}^{(0)}=-50\right): \widehat{C}_{h h}$

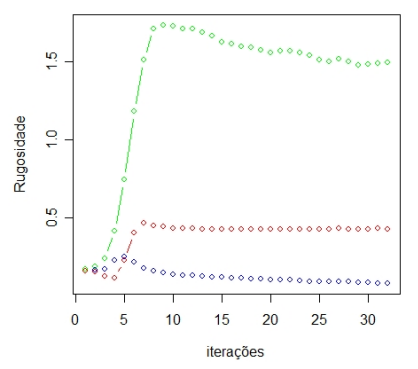

(g) $\mathcal{K}_{P}: \widehat{\boldsymbol{\alpha}}$

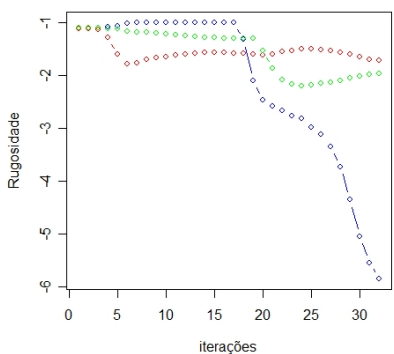

(k) $\mathcal{G}_{P}^{0}: \widehat{\boldsymbol{\alpha}}$

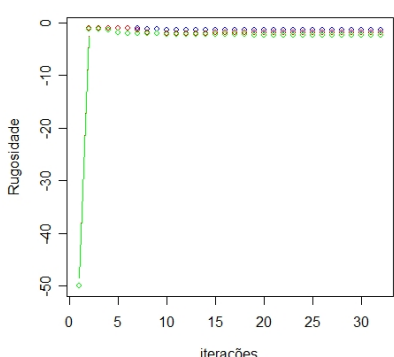

(o) $\mathcal{G}_{P}^{0}\left(\hat{\alpha}^{(0)}=-50\right): \hat{\alpha}$

Figura 17. Curvas de convergência para os parâmetros $\left(\ell(\widehat{\psi})\right.$ - log-verossimilhança, $\widehat{\boldsymbol{\rho}}$-proporções, $\widehat{\boldsymbol{C}}_{h h}$ - intensidade de $\widehat{C}$ na polarização $h h, \widehat{\boldsymbol{\alpha}}$ - rugosidade) com as três regiões (urbano - vermelho, vegetação - verde e oceano - azul) utilizando os resultados com a inicialização aleatória. 


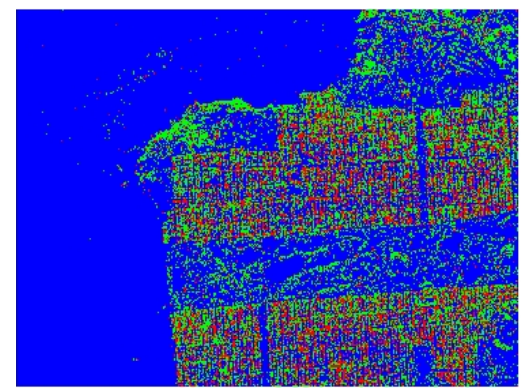

(a) $W$ - iteração 1

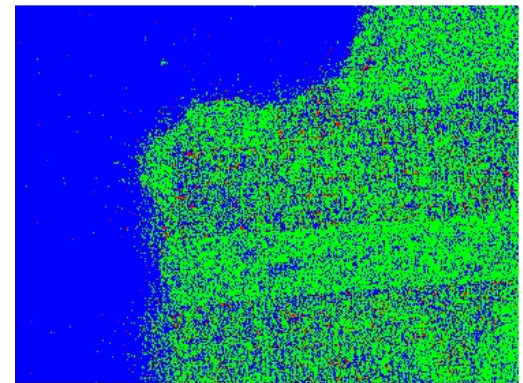

(d) $\mathcal{K}_{P}$ - iteração 1

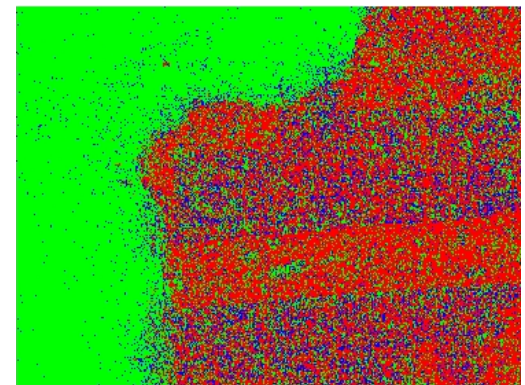

(g) $\mathcal{G}_{P}^{0}$ - iteração 1

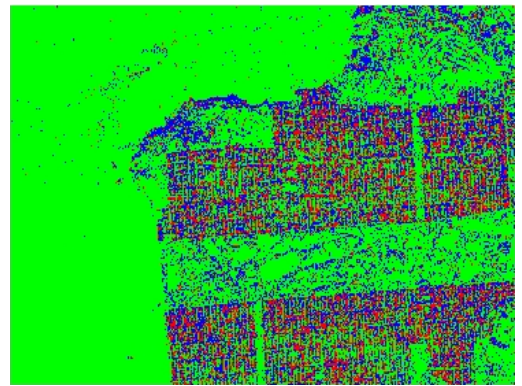

(j) $\mathcal{G}_{P}^{0}(\alpha=-50)$ - iteração 1

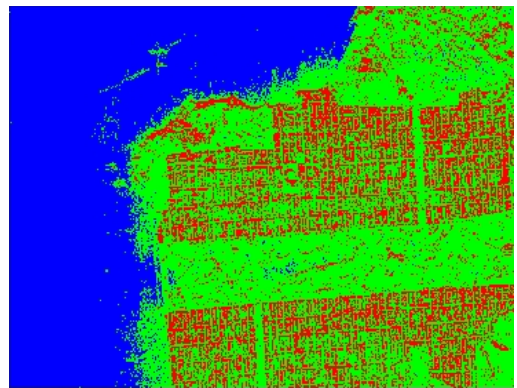

(b) $W$ - iteração 17

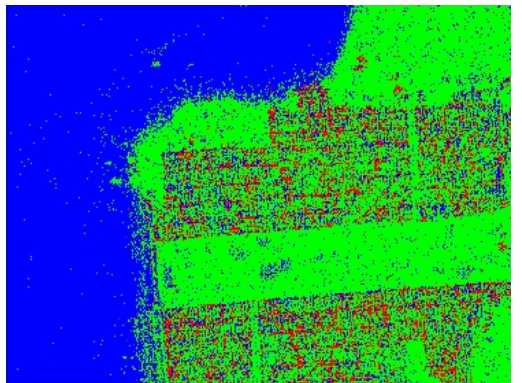

(e) $\mathcal{K}_{P}$ - iteração 17

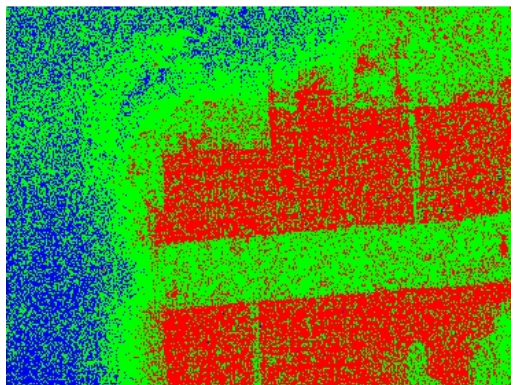

(h) $\mathcal{G}_{P}^{0}$ - iteração 18

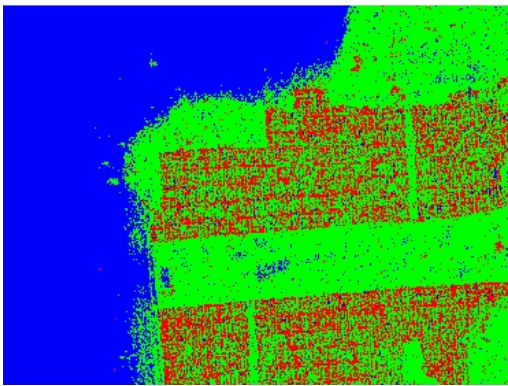

(k) $\mathcal{G}_{P}^{0}(\alpha=-50)$ - iteração 22

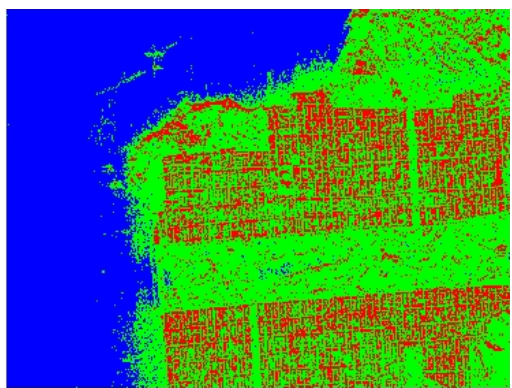

(c) $W$ - iteração 32

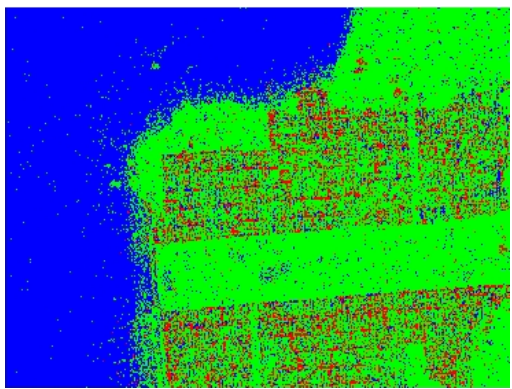

(f) $\mathcal{K}_{P}$ - iteração 32

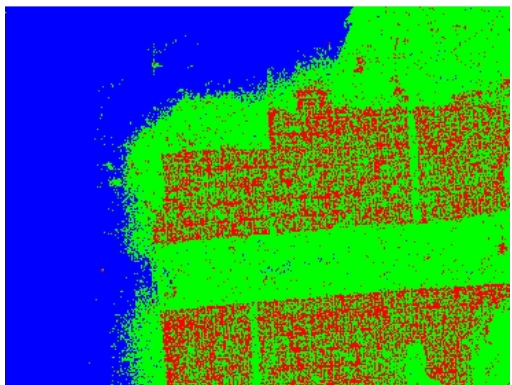

(i) $\mathcal{G}_{P}^{0}$ - iteração 32

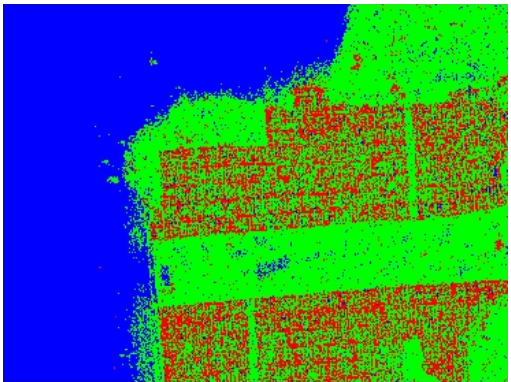

(l) $\mathcal{G}_{P}^{0}(\alpha=-50)$ - iteração 32

Figura 18. Segmentações da imagem São Francisco com $g=3$ regiões utilizando a inicialização aleatória em diferentes iterações. 


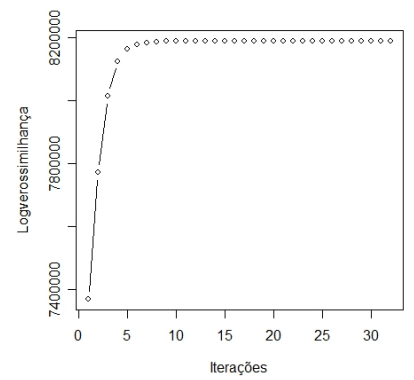

(a) $W: \ell(\widehat{\psi})$

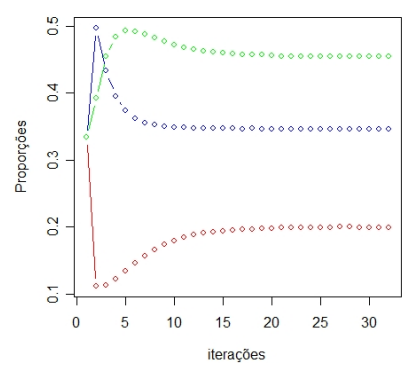

(b) $W: \widehat{\rho}$

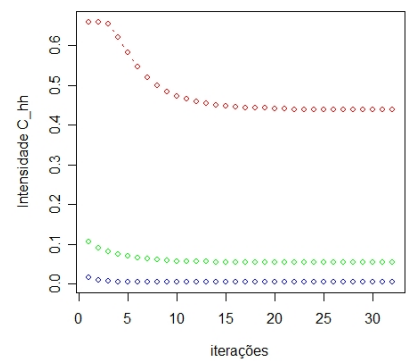

(c) $W: \widehat{C}_{h h}$

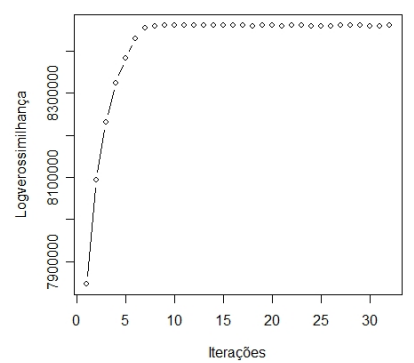

(d) $\mathcal{K}_{P}: \ell(\widehat{\psi})$

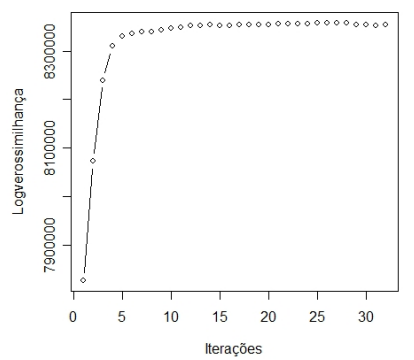

(h) $\mathcal{G}_{P}^{0}: \ell(\widehat{\psi})$

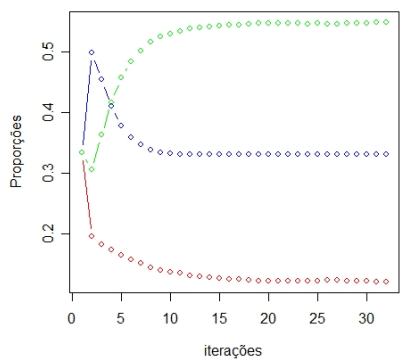

(e) $\mathcal{K}_{P}: \hat{\boldsymbol{\rho}}$

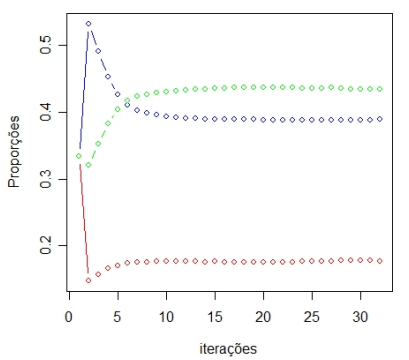

(i) $\mathcal{G}_{P}^{0}: \widehat{\rho}$

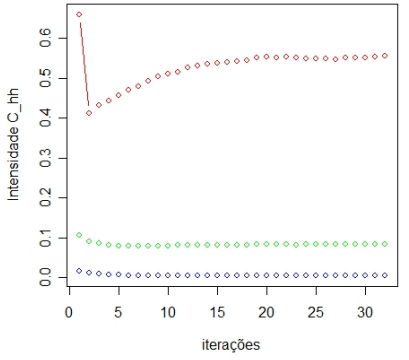

(f) $\mathcal{K}_{P}: \widehat{C}_{h h}$

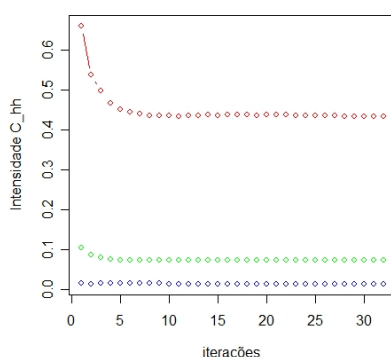

(j) $\mathcal{G}_{P}^{0}: \widehat{\boldsymbol{C}}_{h h}$

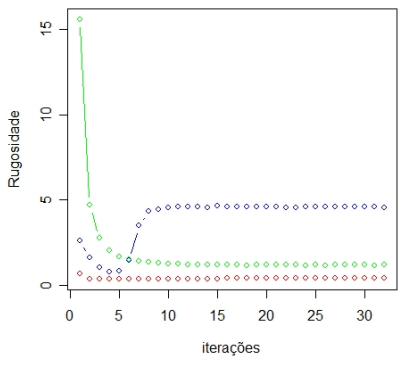

(g) $\mathcal{K}_{P}: \widehat{\boldsymbol{\alpha}}$

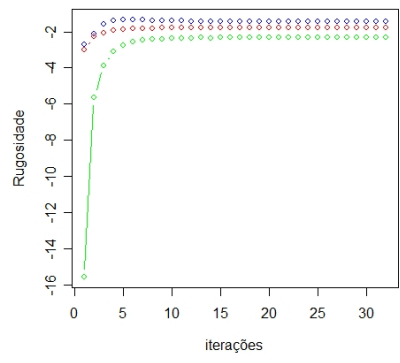

(k) $\mathcal{G}_{P}^{0}: \widehat{\boldsymbol{\alpha}}$

Figura 19. Curvas de convergência para os parâmetros $\left(\ell(\widehat{\psi})\right.$ - log-verossimilhança, $\widehat{\boldsymbol{\rho}}$-proporções, $\widehat{\boldsymbol{C}}_{h h}$ - intensidade de $\widehat{C}$ na polarização $h h, \widehat{\boldsymbol{\alpha}}$ - rugosidade) com as três regiões (urbano - vermelho, vegetação - verde e oceano - azul) utilizando os resultados com a inicialização k-médias. 


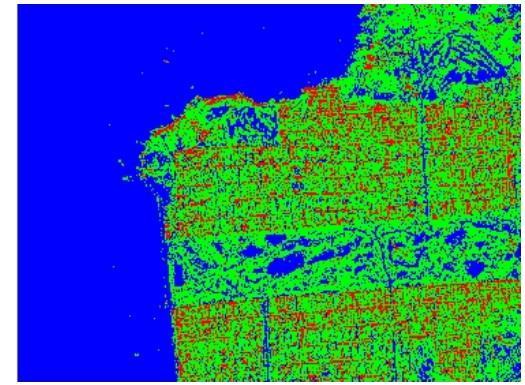

(a) $W$ - iteração 1

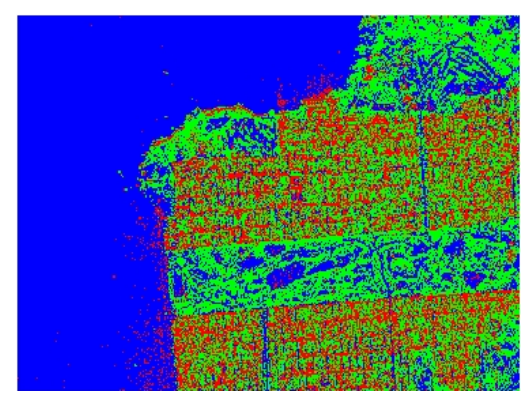

(d) $\mathcal{K}_{P}$ - iteração 1

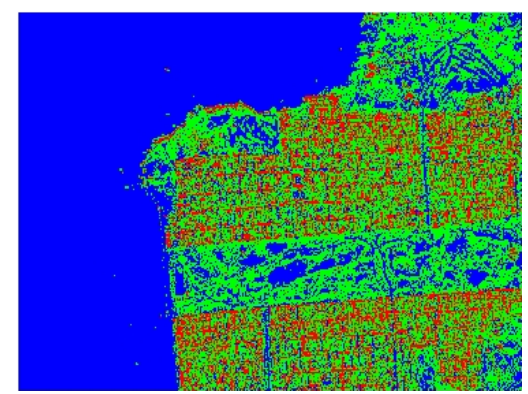

(g) $\mathcal{G}_{P}^{0}$ - iteração 1

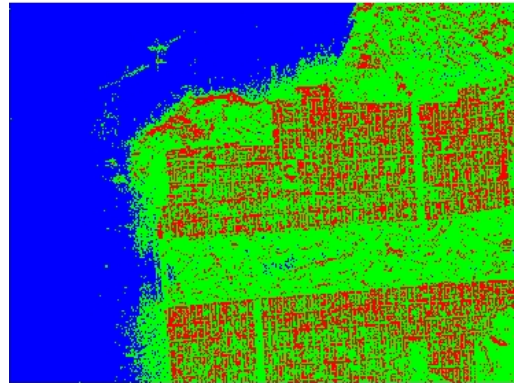

(b) $W$ - iteração 14

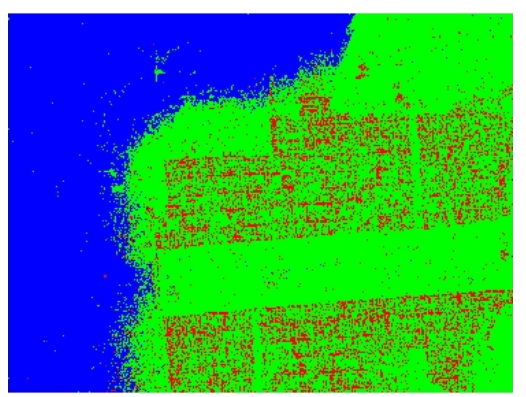

(e) $\mathcal{K}_{P}$ - iteração 13

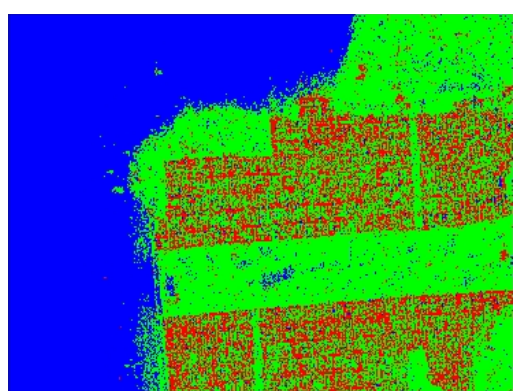

(h) $\mathcal{G}_{P}^{0}$ - iteração 11

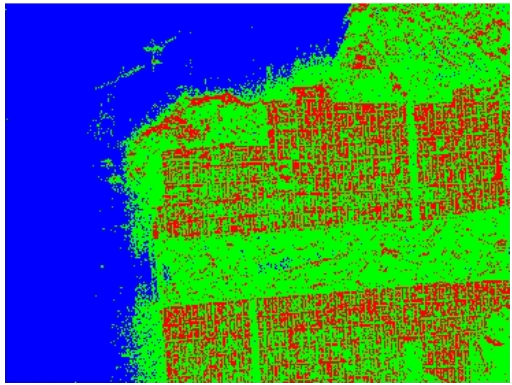

(c) $W$ - iteração 32

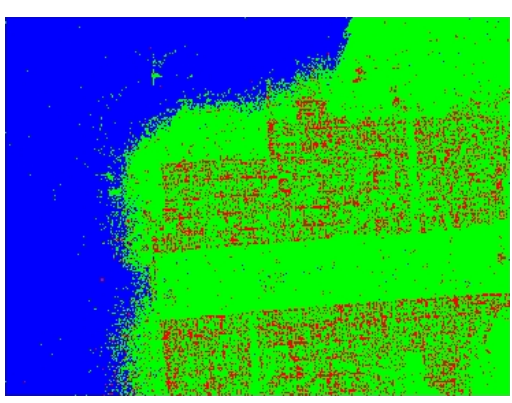

(f) $\mathcal{K}_{P}$ - iteração 32

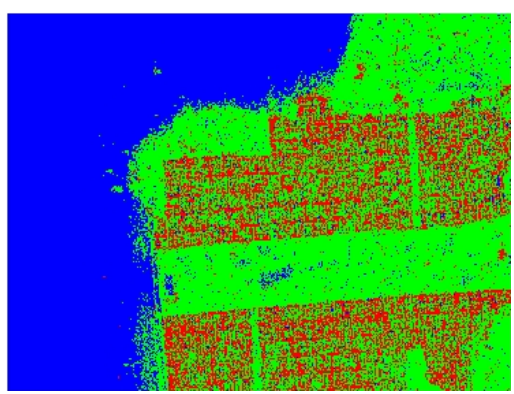

(i) $\mathcal{G}_{P}^{0}$ - iteração 32

Figura 20. Segmentações da imagem São Francisco com $g=3$ regiões utilizando a inicialização k-médias em diferentes iterações. 


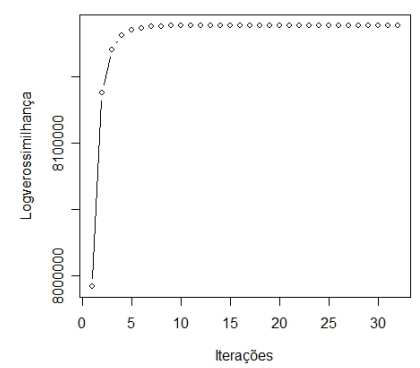

(a) $W: \ell(\widehat{\psi})$

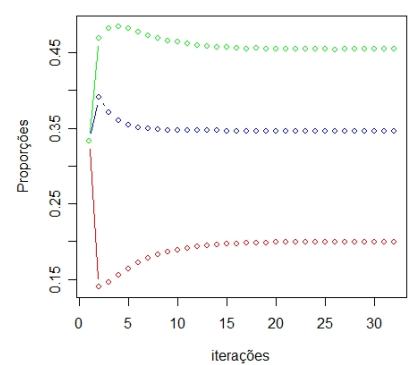

(b) $W: \widehat{\rho}$

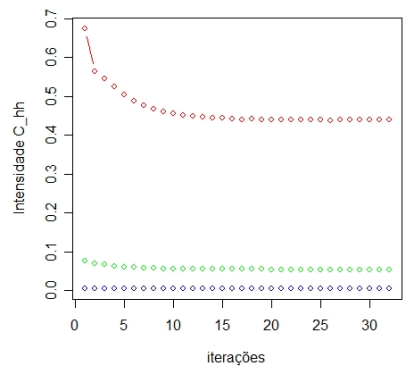

(c) $W: \widehat{C}_{h h}$

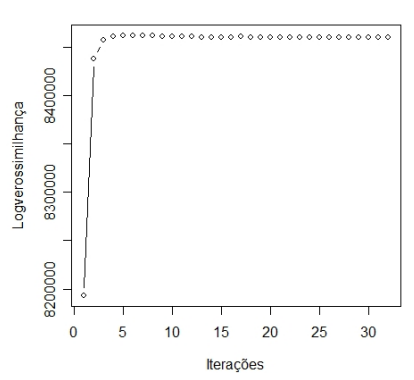

(d) $\mathcal{K}_{P}: \ell(\widehat{\psi})$

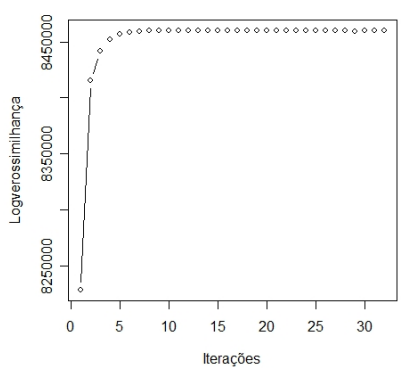

(h) $\mathcal{G}_{P}^{0}: \ell(\widehat{\psi})$

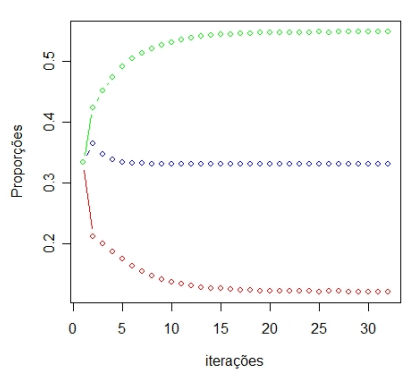

(e) $\mathcal{K}_{P}: \widehat{\rho}$

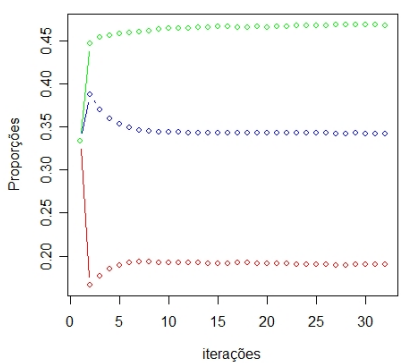

(i) $\mathcal{G}_{P}^{0}: \widehat{\boldsymbol{\rho}}$

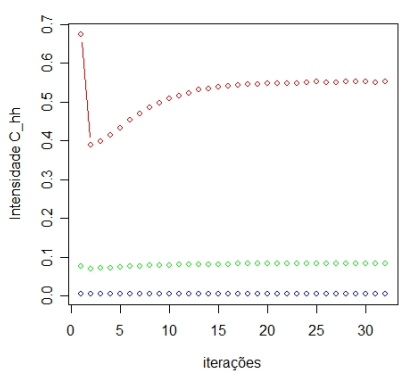

(f) $\mathcal{K}_{P}: \widehat{\boldsymbol{C}}_{h h}$

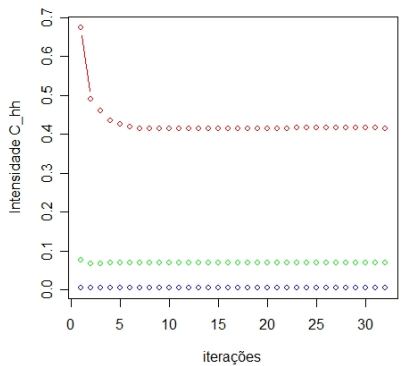

(j) $\mathcal{G}_{P}^{0}: \widehat{\boldsymbol{C}}_{h h}$

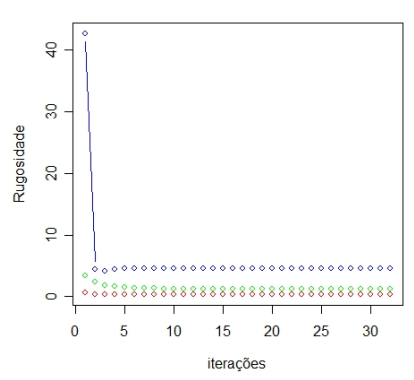

(g) $\mathcal{K}_{P}: \widehat{\boldsymbol{\alpha}}$

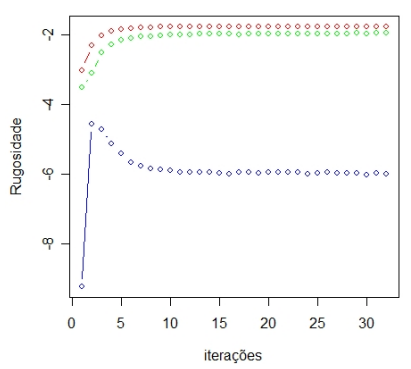

(k) $\mathcal{G}_{P}^{0}: \widehat{\boldsymbol{\alpha}}$

Figura 21. Curvas de convergência para os parâmetros $\left(\ell(\widehat{\psi})\right.$ - log-verossimilhança, $\widehat{\boldsymbol{\rho}}$-proporções, $\widehat{\boldsymbol{C}}_{h h}$ - intensidade de $\widehat{C}$ na polarização $h h, \widehat{\boldsymbol{\alpha}}$ - rugosidade) com as três regiões (urbano - vermelho, vegetação - verde e oceano - azul) utilizando os resultados com a inicialização quantil. 


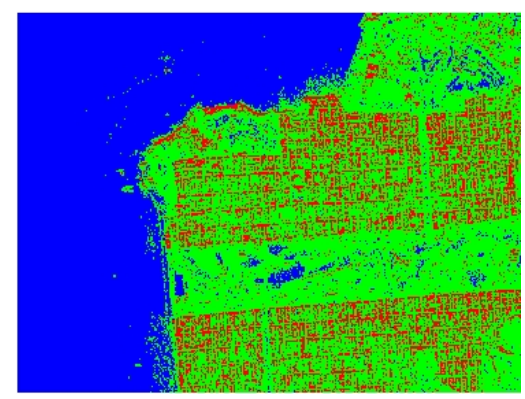

(a) $W$ - iteração 1

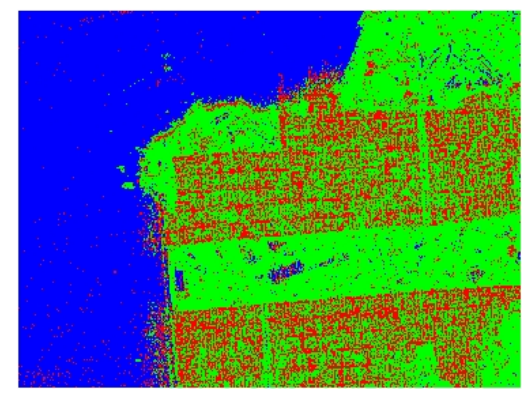

(d) $\mathcal{K}_{P}$ - iteração 1

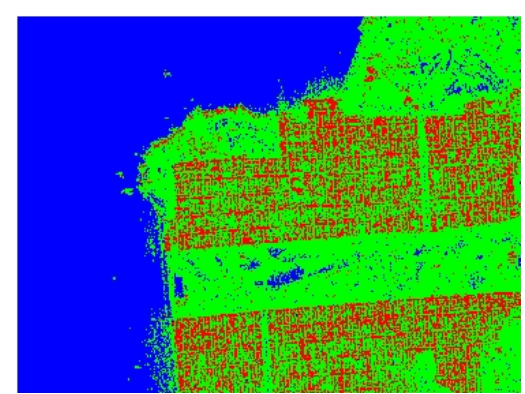

(g) $\mathcal{G}_{P}^{0}$ - iteração 1

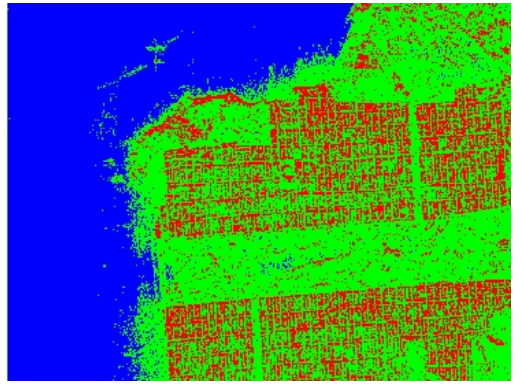

(b) $W$ - iteração 10

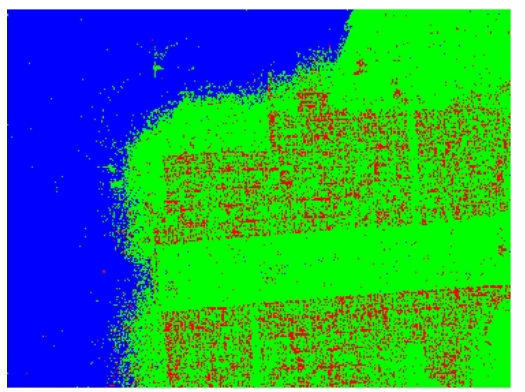

(e) $\mathcal{K}_{P}$ - iteração 14

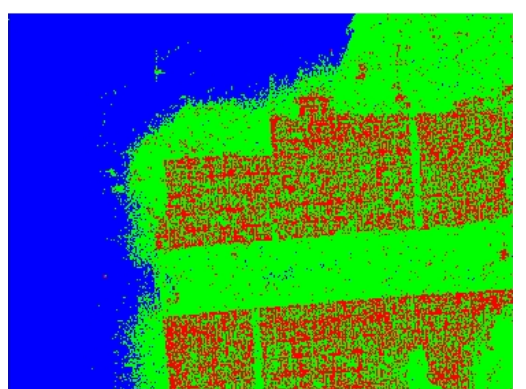

(h) $\mathcal{G}_{P}^{0}$ - iteração 8

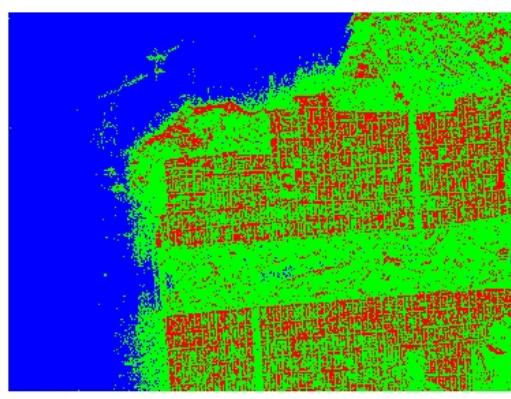

(c) $W$ - iteração 32

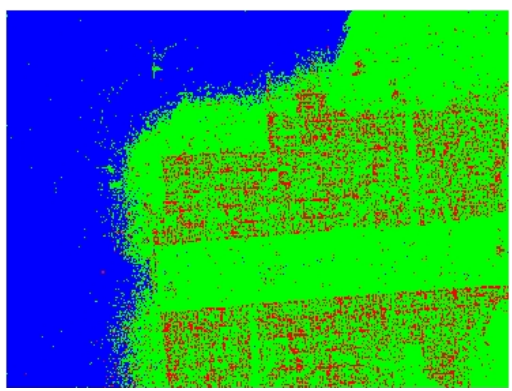

(f) $\mathcal{K}_{P}$ - iteração 32

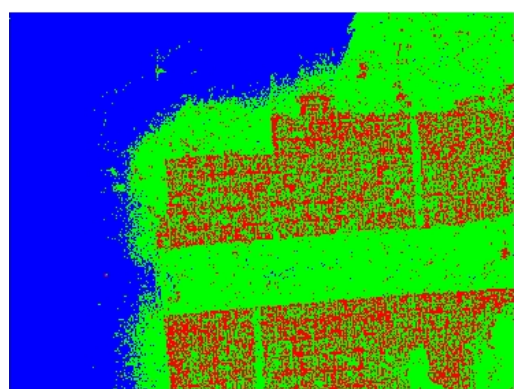

(i) $\mathcal{G}_{P}^{0}$ - iteração 32

Figura 22. Segmentações da imagem São Francisco com $g=3$ regiões utilizando a inicialização quantil em diferentes iterações. 


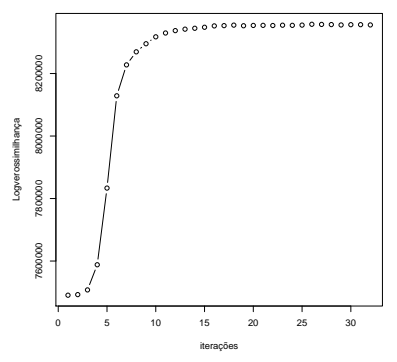

(a) Aleatória: $\ell(\widehat{\psi})$

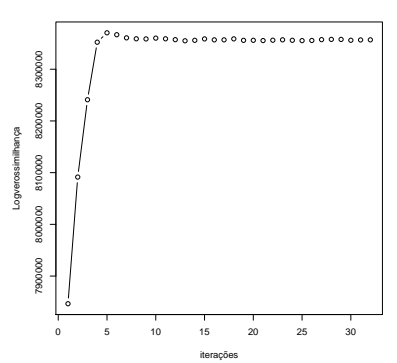

(e) k-médias: $\ell(\widehat{\psi})$

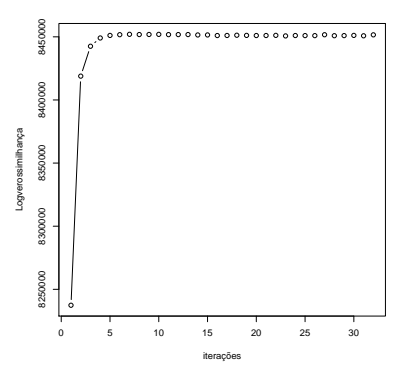

(i) Quantil: $\ell(\widehat{\psi})$

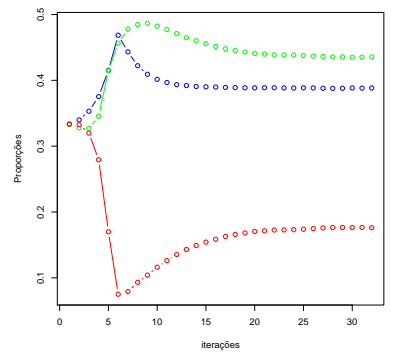

(b) Aleatória: $\widehat{\rho}$

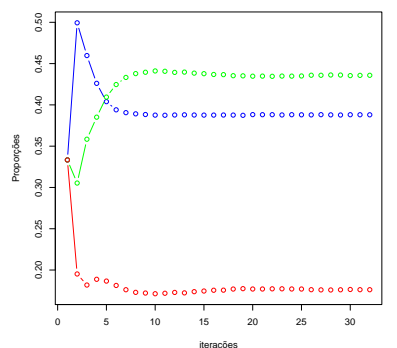

(f) k-médias: $\widehat{\rho}$

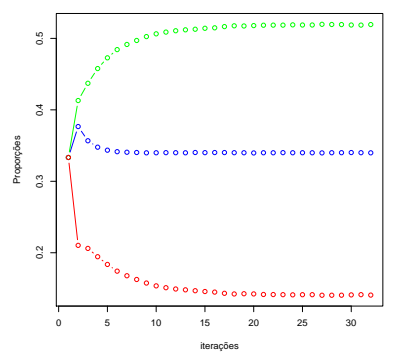

(j) Quantil: $\widehat{\rho}$

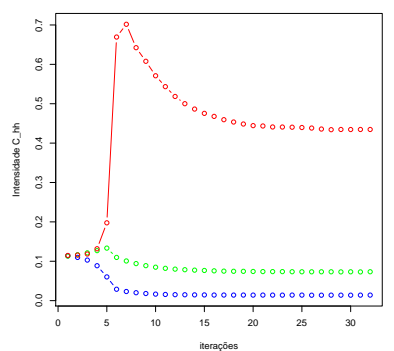

(c) Aleatória: $\widehat{C}_{h h}$

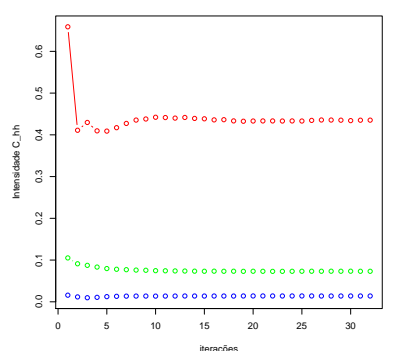

(g) k-médias: $\widehat{C}_{h h}$

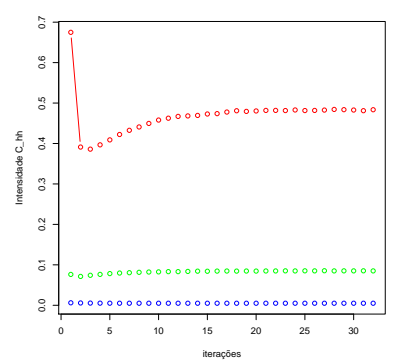

(k) Quantil: $\widehat{\boldsymbol{C}}_{h h}$

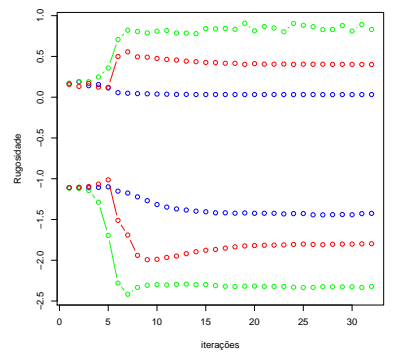

(d) Aletória: $\widehat{\alpha}$

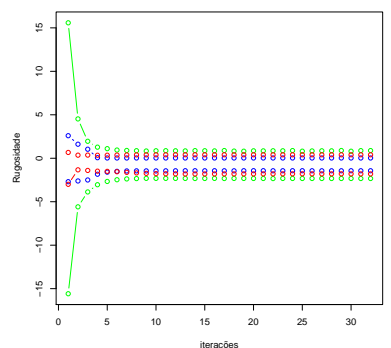

(h) k-médias: $\widehat{\alpha}$

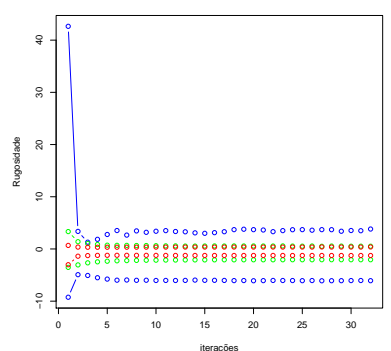

(l) quantil: $\widehat{\boldsymbol{\alpha}}$

Figura 23. Curvas de convergência para os parâmetros $\left(\ell(\widehat{\psi})\right.$ - log-verossimilhança, $\widehat{\boldsymbol{\rho}}$-proporções, $\widehat{\boldsymbol{C}}_{h h}$ - intensidade de $\widehat{C}$ na polarização $h h, \widehat{\boldsymbol{\alpha}}$ - rugosidade) com as três regiões (urbano - vermelho, vegetação - verde e oceano - azul) utilizando os resultados do método SEM com modelo de mistura do dicionário de famílias. 


\section{APÊNDICE C - Resultados da Imagem Munique}

Este apêndice apresenta os resultados obtidos com o método SEM dos modelos de mistura $\mathcal{G}_{P}^{0}, \mathcal{K}_{P}$ e Wishart utilizando a inicialização quantil.

As Figuras 24 e 26 ilustram as curvas de convergência da log-verossimilhança $(\ell(\widehat{\psi}))$ e dos parâmetros dos modelos de mistura $(\widehat{\psi})$ das distribuições Wishart, $\mathcal{K}_{P}$ e $\mathcal{G}_{P}^{0}$. Na Figura 26 há a seleção de amostras.

As Figuras 25 e 27 ilustram as imagens segmentadas. Na Figura 27 há a seleção de amostras. 


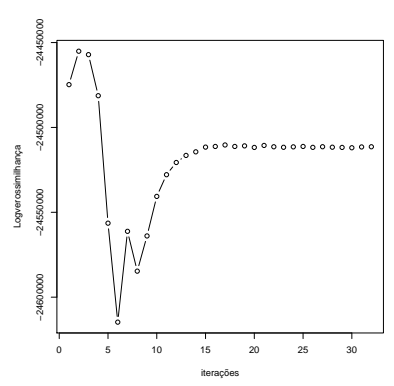

(a) $\mathcal{G}_{P}^{0}: \ell(\widehat{\psi})$

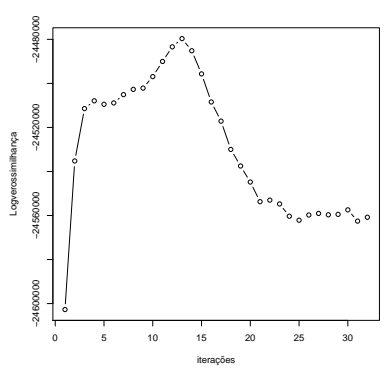

(e) $\mathcal{K}_{P}: \ell(\widehat{\psi})$

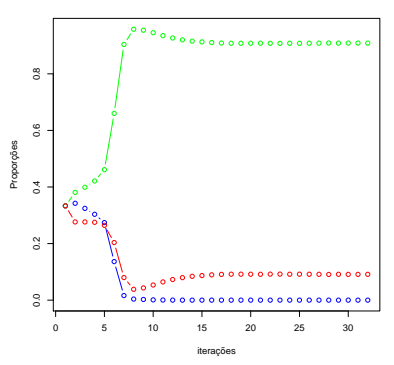

(b) $\mathcal{G}_{P}^{0}: \widehat{\rho}$

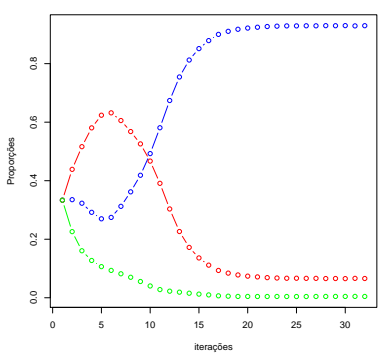

(f) $\mathcal{K}_{P}: \widehat{\rho}$

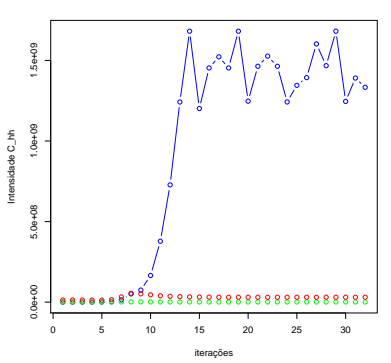

(c) $\mathcal{G}_{P}^{0}: \widehat{\boldsymbol{C}}_{h h}$

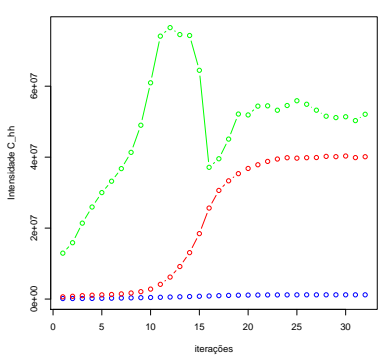

(g) $\mathcal{K}_{P}: \widehat{C}_{h h}$

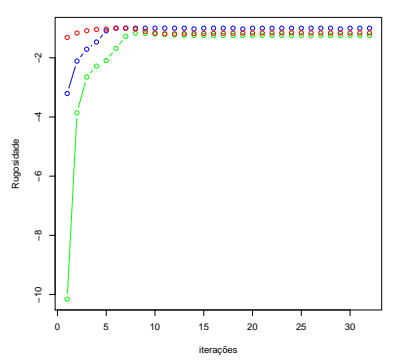

(d) $\mathcal{G}_{P}^{0}: \widehat{\boldsymbol{\alpha}}$

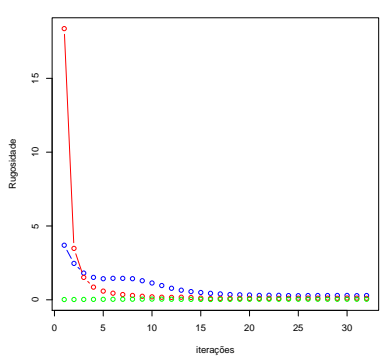

(h) $\mathcal{K}_{P}: \widehat{\alpha}$

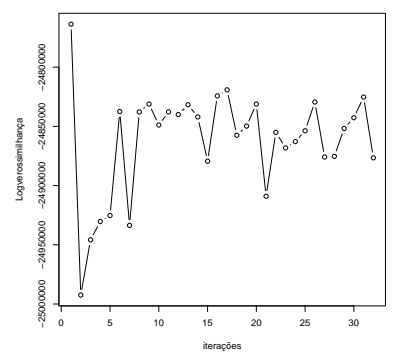

(i) $\operatorname{SEM} W: \ell(\widehat{\psi})$

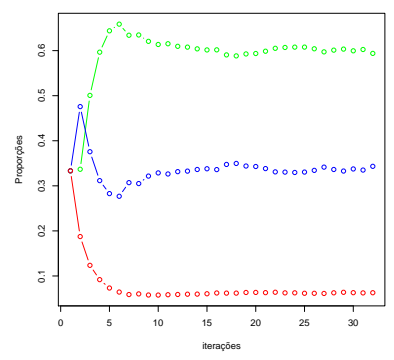

(j) SEM $W: \widehat{\rho}$

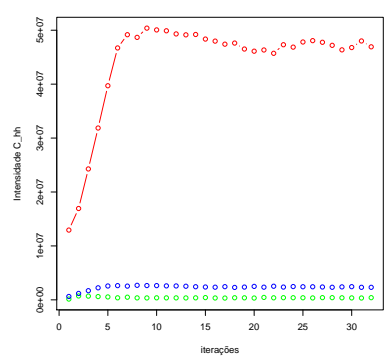

(k) SEM $W: \widehat{C}_{h h}$

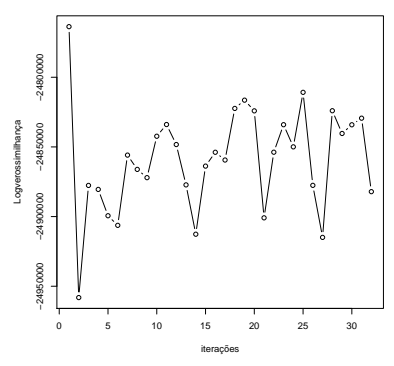

(l) EM - $W: \ell(\widehat{\psi})$

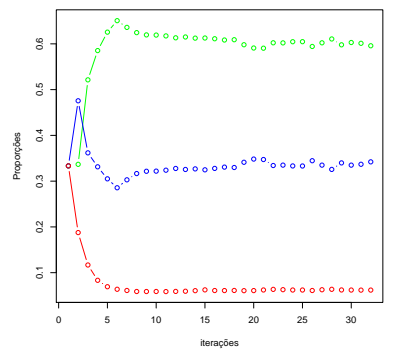

(m) EM $W: \widehat{\rho}$

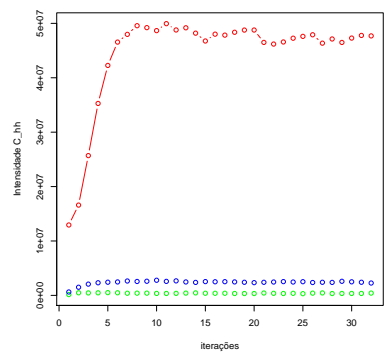

(n) EM $-W: \widehat{C}_{h h}$

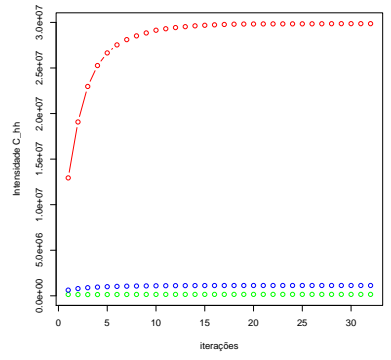

(o) k-médias - $W: \widehat{C}_{h h}$

Figura 24. Curvas de convergência dos parâmetros da imagem de Munique completa $(\ell(\widehat{\psi})$ - logverossimilhança, $\widehat{\boldsymbol{\rho}}$-proporções, $\widehat{C}_{h h}$ - intensidade de $\widehat{C}$ na polarização $h h, \widehat{\alpha}$ - rugosidade) com as três regiões (urbano - vermelho, vegetação - azul e cultivo - verde) e os métodos SEM, EM e k-médias inicializados com o método quantil. 


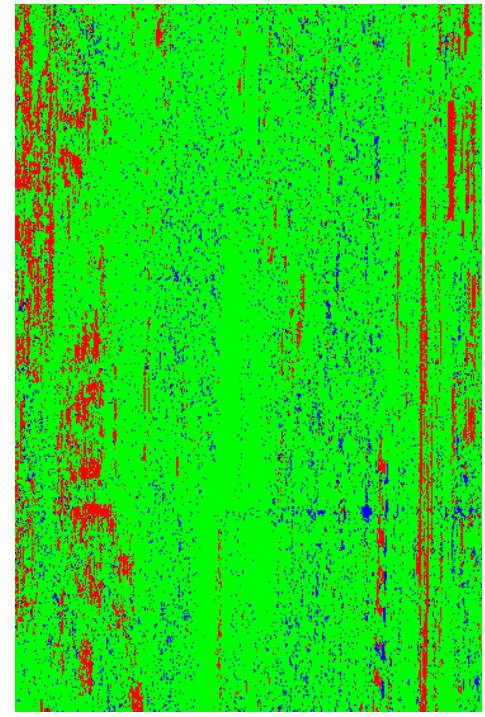

(a) $\operatorname{SEM} \mathcal{G}_{P}^{0}$

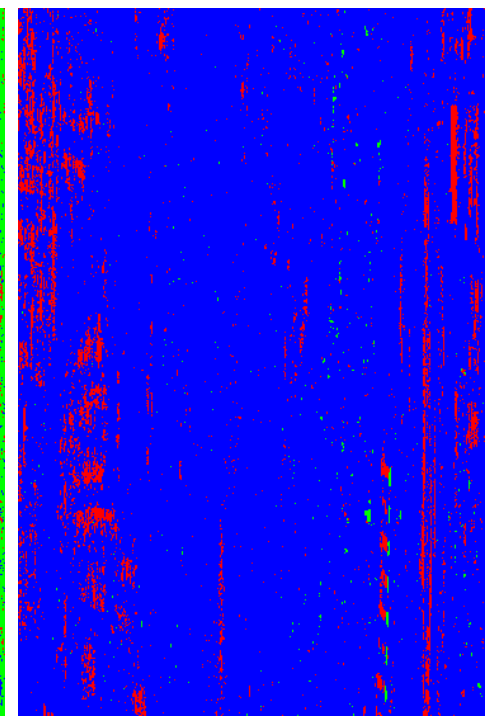

(b) $\operatorname{SEM} \mathcal{K}_{P}$

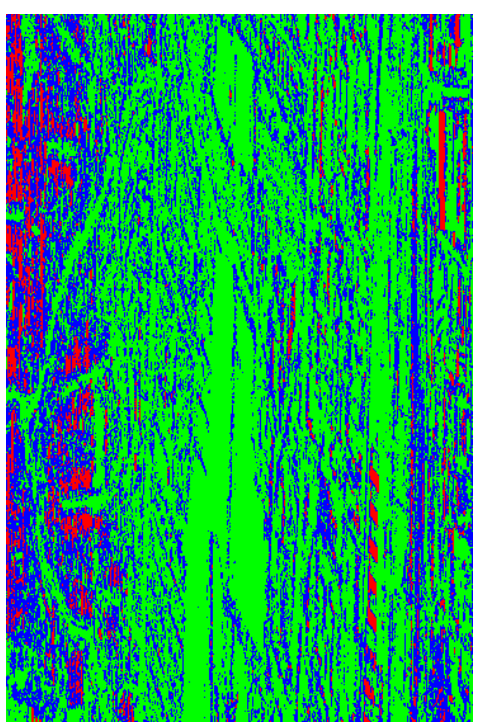

(c) SEM $W$

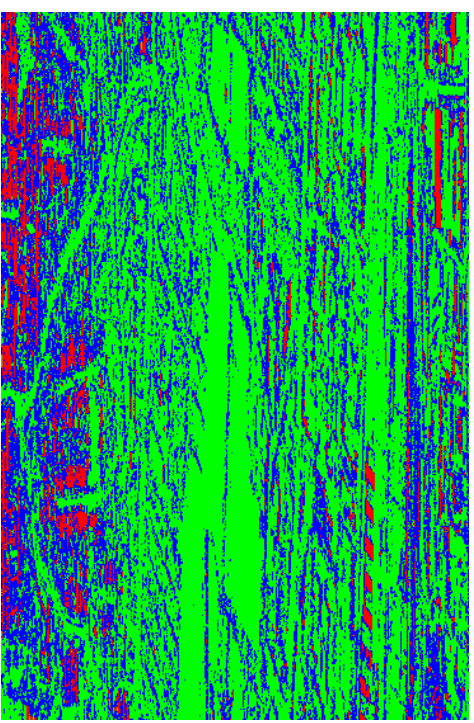

(d) EM $W$

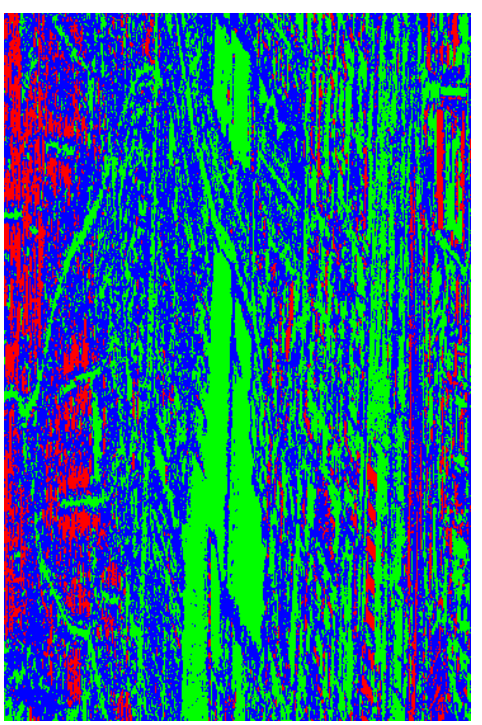

(e) k-médias

Figura 25. Segmentações da imagem Munique completa com $g=3$ regiões utilizando a inicialização quantil na iteração 32 . 


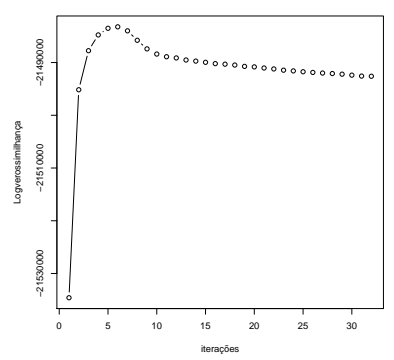

(a) $\mathcal{G}_{P}^{0}: \ell(\widehat{\psi})$

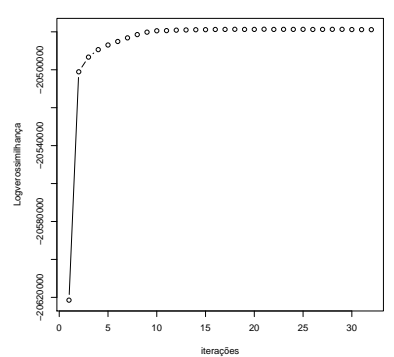

(e) $\mathcal{K}_{P}: \ell(\widehat{\psi})$

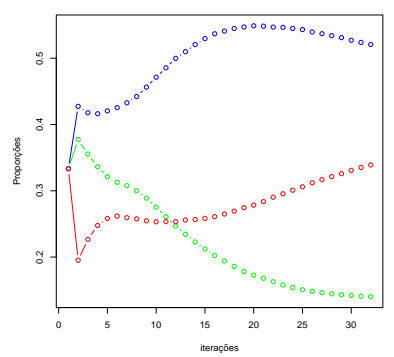

(b) $\mathcal{G}_{P}^{0}: \widehat{\rho}$

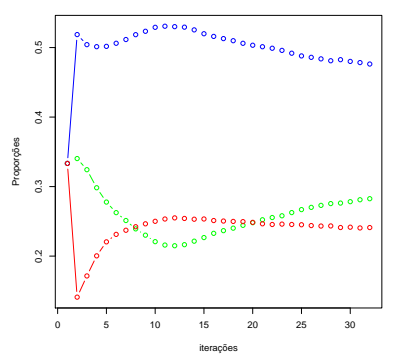

(f) $\mathcal{K}_{P}: \widehat{\rho}$

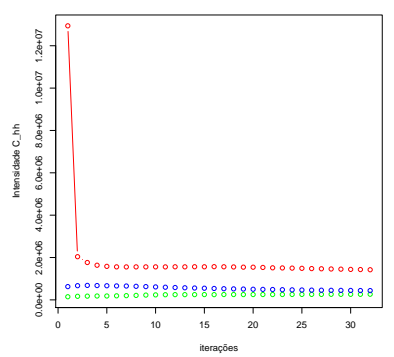

(c) $\mathcal{G}_{P}^{0}: \widehat{\boldsymbol{C}}_{h h}$

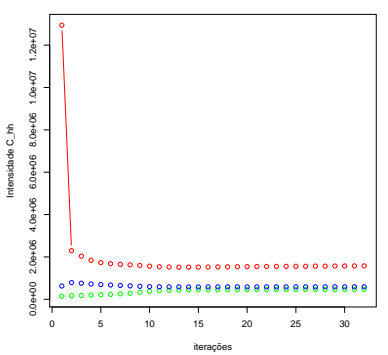

(g) $\mathcal{K}_{P}: \widehat{C}_{h h}$

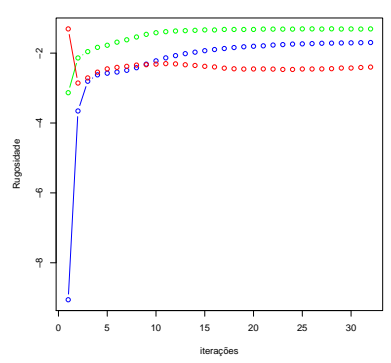

(d) $\mathcal{G}_{P}^{0}: \widehat{\boldsymbol{\alpha}}$

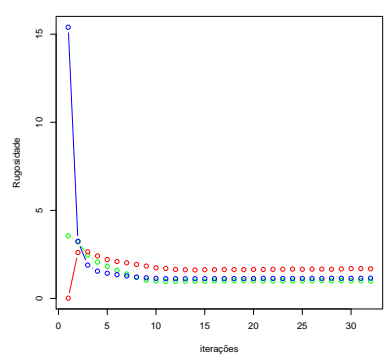

(h) $\mathcal{K}_{P}: \widehat{\boldsymbol{\alpha}}$

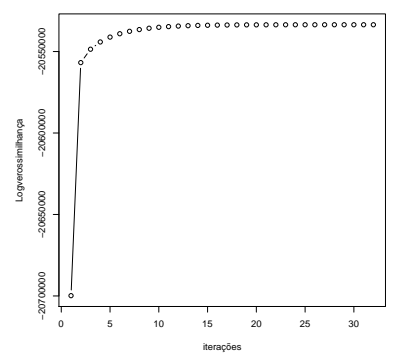

(i) $\operatorname{SEM} W: \ell(\widehat{\psi})$

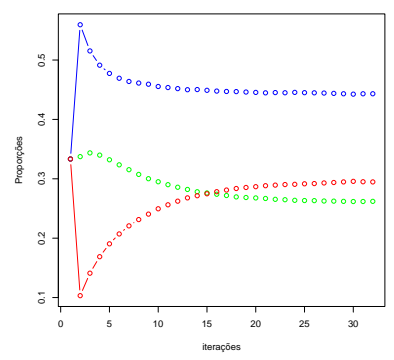

(j) SEM $W: \widehat{\rho}$

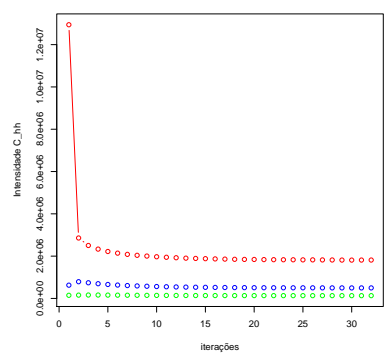

(k) SEM $W: \widehat{C}_{h h}$

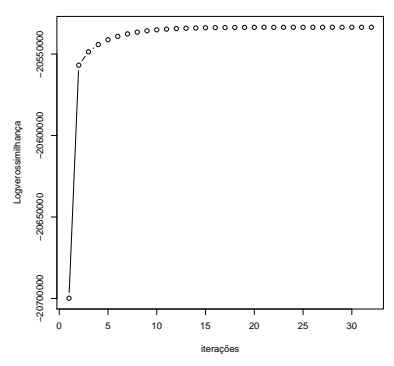

(l) EM $-W: \ell(\widehat{\psi})$

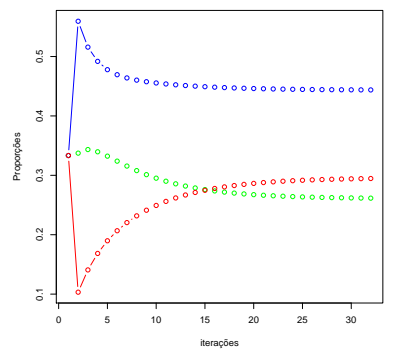

(m) EM $W: \widehat{\rho}$

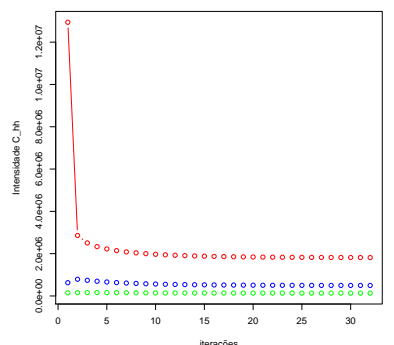

(n) EM $-W: \widehat{C}_{h h}$

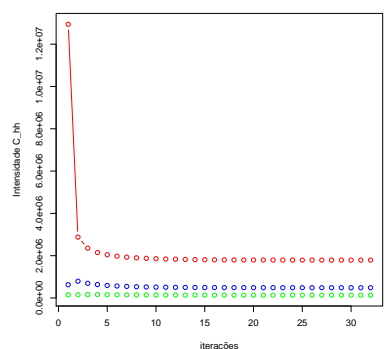

(o) k-médias - $W: \widehat{C}_{h h}$

Figura 26. Curvas de convergência dos parâmetros da imagem de Munique parcial $(\ell(\widehat{\psi})-\log$ verossimilhança, $\widehat{\boldsymbol{\rho}}$-proporções, $\widehat{\boldsymbol{C}}_{h h}$ - intensidade de $\widehat{C}$ na polarização $h h, \widehat{\boldsymbol{\alpha}}$ - rugosidade) com as três regiões (urbano - vermelho, vegetação - azul e cultivo - verde) e os métodos SEM, EM e k-médias inicializados com o método quantil. Os métodos foram processados com $90 \%$ dos pixels da imagem, considerou-se os pixels da imagem com $S P A N \in\left[Q_{0,05}, Q_{0,95}\right]$. 


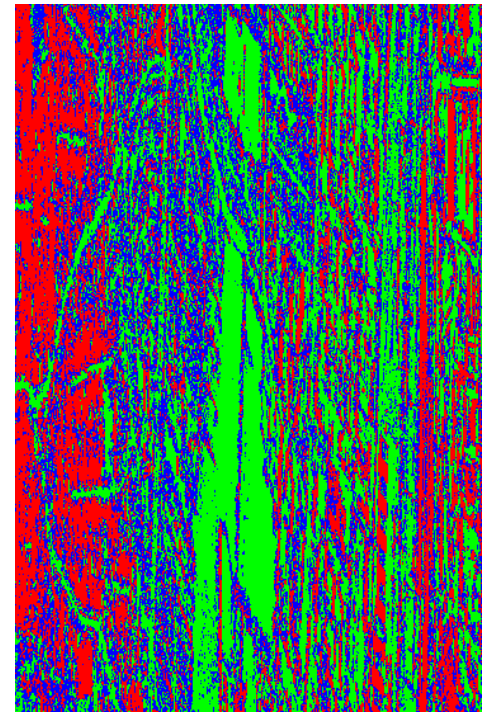

(a) Inicialização Quantil

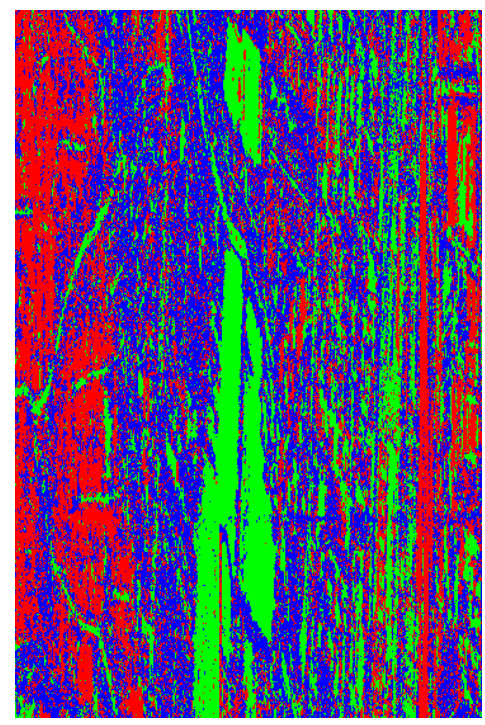

(c) SEM $\mathcal{G}_{P}^{0}$ - iteração 6

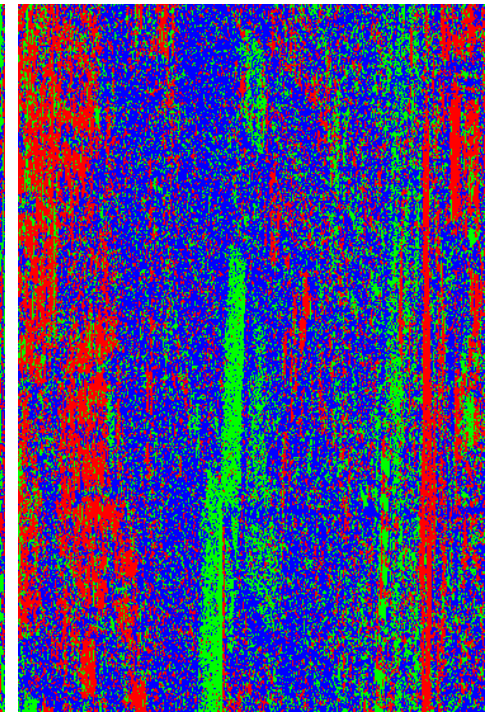

(b) SEM $\mathcal{K}_{P}$ - iteração 20

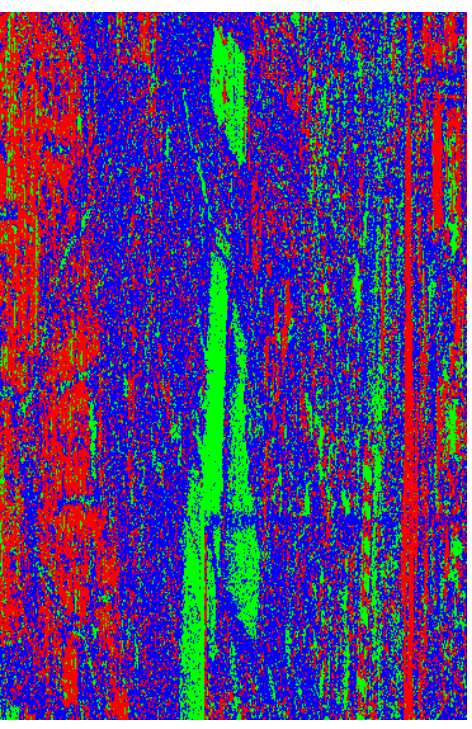

(d) SEM $\mathcal{G}_{P}^{0}$ - iteração 32

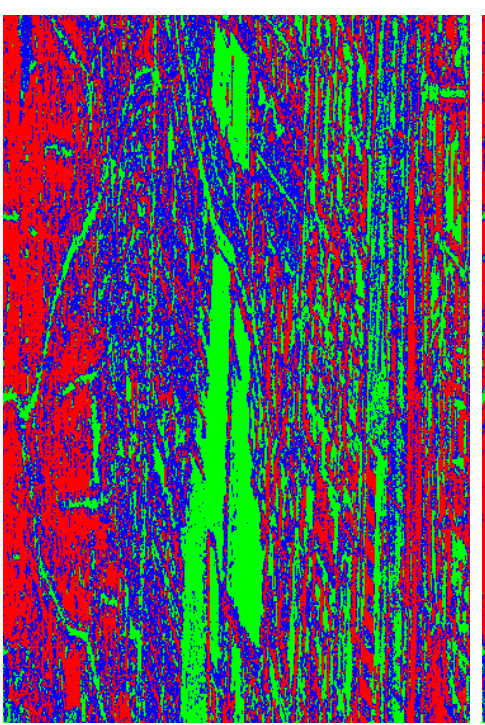

(e) SEM $W$ - iteração 32

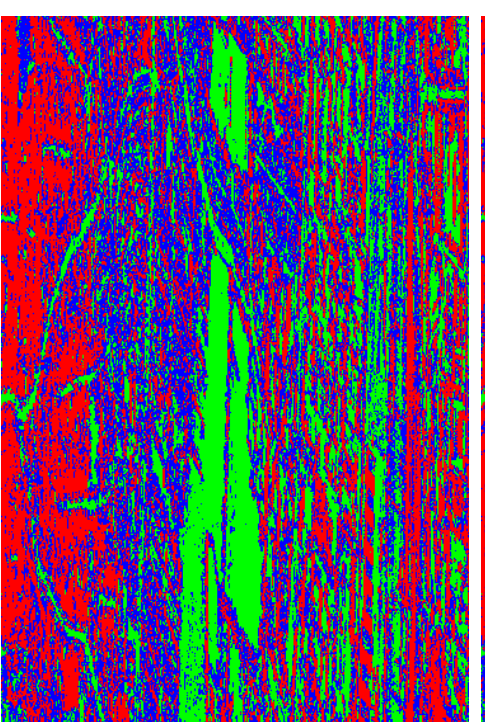

(f) EM $W$ - iteração 32

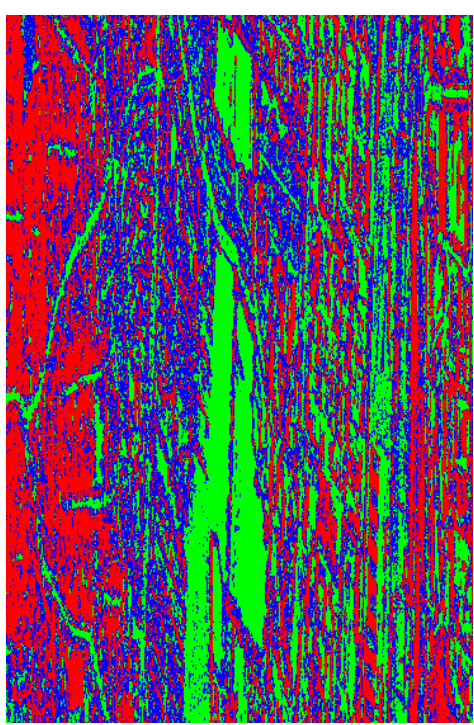

(g) k-médias $W$ - iteração 10

Figura 27. Segmentações da imagem Munique completa com $g=3$ regiões utilizando a inicialização quantil. Os métodos foram processados com $90 \%$ dos pixels da imagem, consideraram-se os pixels da imagem com $S P A N \in\left[Q_{0,05}, Q_{0,95}\right]$ 